\title{
Homogeneous M2 duals
}

\author{
José Figueroa-O'Farrill ${ }^{a}$ and Mara Ungureanu ${ }^{b}$ \\ ${ }^{a}$ School of Mathematics and Maxwell Institute for Mathematical Sciences, \\ The University of Edinburgh, \\ James Clerk Maxwell Building, The King's Buildings, Peter Guthrie Tait Road, \\ Edinburgh EH9 3FD, Scotland, U.K. \\ ${ }^{b}$ Humboldt-Universität zu Berlin, Institut für Mathematik, \\ Unter den Linden 6, 10099 Berlin, Germany \\ E-mail: J.M.Figueroa@ed.ac.uk, ungurean@math.hu-berlin.de
}

Abstract: Motivated by the search for new gravity duals to M2 branes with $N>4$ supersymmetry - equivalently, M-theory backgrounds with Killing superalgebra $\mathfrak{o s p}(N \mid 4)$ for $N>4$ - we classify (except for a small gap) homogeneous M-theory backgrounds with symmetry Lie algebra $\mathfrak{s o}(n) \oplus \mathfrak{s o}(3,2)$ for $n=5,6,7$. We find that there are no new backgrounds with $n=6,7$ but we do find a number of new (to us) backgrounds with $n=5$. All backgrounds are metrically products of the form $\mathrm{AdS}_{4} \times P^{7}$, with $P$ riemannian and homogeneous under the action of $\mathrm{SO}(5)$, or $S^{4} \times Q^{7}$ with $Q$ lorentzian and homogeneous under the action of $\mathrm{SO}(3,2)$. At least one of the new backgrounds is supersymmetric (albeit with only $N=2$ ) and we show that it can be constructed from a supersymmetric Freund-Rubin background via a Wick rotation. Two of the new backgrounds have only been approximated numerically.

KEYWORDS: Gauge-gravity correspondence, Differential and Algebraic Geometry, Supergravity Models

ARXIV EPRINT: 1511.03637 


\section{Contents}

1 Introduction 1

2 Homogeneous lorentzian manifolds $\quad 3$

2.1 Basic notions about homogeneous spaces 3

$\begin{array}{ll}2.2 \text { Computations in homogeneous spaces } & 6\end{array}$

$\begin{array}{lll}3 & \text { Lie subalgebras of a direct product } & 9\end{array}$

4 Lie subalgebras of $\mathfrak{s o}(n) \quad 11$

$\begin{array}{lll}4.1 & \text { Lie subalgebras of } \mathfrak{s o}(2) & 11\end{array}$

$\begin{array}{lll}4.2 & \text { Lie subalgebras of } \mathfrak{s o}(3) & 11\end{array}$

$\begin{array}{lll}4.3 & \text { Lie subalgebras of } \mathfrak{s o}(4) & 12\end{array}$

$\begin{array}{lll}4.4 & \text { Lie subalgebras of } \mathfrak{s o}(5) & 13\end{array}$

$\begin{array}{lll}4.5 & \text { Lie subalgebras of } \mathfrak{s o}(6) & 14\end{array}$

$\begin{array}{lll}4.6 & \text { Lie subalgebras of } \mathfrak{s o}(7) & 18\end{array}$

5 The supergravity field equations for homogeneous backgrounds $\quad 18$

$\begin{array}{ll}5.1 \text { The field equations of eleven-dimensional supergravity } & 19\end{array}$

$\begin{array}{lll}5.2 & \text { The equivalent algebraic equations } & 19\end{array}$

$\begin{array}{ll}5.3 \text { The methodology } & 20\end{array}$

6 Homogeneous non-AdS backgrounds $\quad 22$

6.1 Still no $n=7$ duals $\quad 22$

6.2 No new $n=6$ duals $\quad 22$

6.3 Possible new $n=5$ duals 23

7 Homogeneous anti de Sitter backgrounds $\quad 28$

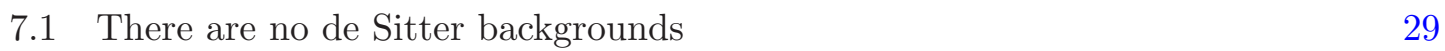

$\begin{array}{lll}7.2 & \text { No new } n=7 \quad \mathrm{AdS}_{4} \text { backgrounds } & 29\end{array}$

7.3 No new $n=6 \quad \mathrm{AdS}_{4}$ backgrounds 31

7.4 Possible new $n=5 \mathrm{AdS}_{4}$ backgrounds 33

$\begin{array}{lll}7.4 .1 & \mathfrak{s o}(3) & \text { irr isotropy }\end{array}$

$\begin{array}{lll}7.4 .2 & \mathfrak{s o}(3) & \text { isotropy }\end{array}$

$\begin{array}{lll}7.4 .3 & \mathfrak{s o}(3)_{+} \text {isotropy } & 36\end{array}$

8 The geometry of some $n=5$ backgrounds $\quad 39$

$\begin{array}{ll}8.1 n=5 \text { anti de Sitter backgrounds } & 39\end{array}$

8.2 Other $n=5$ backgrounds 43

8.2.1 A supersymmetric Freund-Rubin background 43

$\begin{array}{ll}\text { 8.2.2 A circle of backgrounds } & 46\end{array}$ 


\section{Introduction}

The gauge/gravity correspondence for M2 branes [1] suggests that with every threedimensional superconformal field theory, there should be associated a supersymmetric background of eleven-dimensional supergravity, whose Killing (or more generally, symmetry) superalgebra is isomorphic to the superconformal algebra of the field theory. It follows from Nahm's classification [2] that the three-dimensional conformal superalgebra is isomorphic to $\mathfrak{o s p}(N \mid 4)$ for some $N \leq 8$. The even subalgebra of $\mathfrak{o s p}(N \mid 4)$ is $\mathfrak{s o}(N) \oplus \mathfrak{s p}(4 ; \mathbb{R})$, where $\mathfrak{s p}(4 ; \mathbb{R}) \cong \mathfrak{s o}(3,2)$ is the conformal algebra of three-dimensional Minkowski spacetime. This Lie algebra is also isomorphic to the isometry algebra of $\mathrm{AdS}_{4}$ of which (the conformal compactification of) Minkowski spacetime is the conformal boundary.

The original observation in [1] makes use of the fact that the near-horizon geometry of the elementary M2-brane solution of eleven-dimensional supergravity [3] is isometric to $\mathrm{AdS}_{4} \times S^{7}$ [4]. This is a maximally supersymmetric background of eleven-dimensional supergravity [5], its Killing superalgebra is isomorphic to $\mathfrak{o s p}(8 \mid 4)$ and hence the dual superconformal field theory has $N=8$ supersymmetry. One can replace $S^{7}$ by other manifolds admitting real Killing spinors and in this way obtain backgrounds with Killing superalgebra $\mathfrak{o s p}(N \mid 4)$ for lower values of $N[6,7]$. Recently the classification of smooth Freund-Rubin backgrounds of the form $\mathrm{AdS}_{4} \times X^{7}$ with $N \geq 4$ has been achieved [8]: they are necessarily such that $X=S^{7} / \Gamma$, where $\Gamma<\operatorname{Spin}(8)$ is a discrete group acting freely on $S^{7}$ and described as the image of a twisted embedding of an ADE subgroup of quaternions. A classification of singular quotients with $N \geq 4$ has also recently been obtained [9], this time in terms of fibered products of ADE subgroups.

The question remains whether there are any eleven-dimensional supergravity backgrounds with Killing superalgebra isomorphic to $\mathfrak{o s p}(N \mid 4)$ but which are not Freund-Rubin backgrounds of the form $\mathrm{AdS}_{4} \times X^{7}$. Classifying such backgrounds would complete the determination of possible dual geometries to three-dimensional superconformal field theories. The purpose of this paper is to investigate their existence.

It has recently been shown [10] that backgrounds preserving more than half of the supersymmetry - i.e., $N>4$ in the present context - are (locally) homogeneous and moreover that it is the group whose Lie algebra is generated by the Killing spinors of the background which already acts transitively. This allows us to restrict ourselves to backgrounds which are homogeneous under a prescribed group. Homogeneous lorentzian manifolds can be described locally by a pair $(\mathfrak{g}, \mathfrak{h})$, where $\mathfrak{h}$ is a Lie subalgebra of $\mathfrak{g}$ preserving a lorentzian inner product on the representation $\mathfrak{g} / \mathfrak{h}$ induced by the restriction to $\mathfrak{h}$ of the adjoint representation of $\mathfrak{g}$. In addition, the group corresponding to $\mathfrak{h}$ must be a closed subgroup of the group corresponding to $\mathfrak{g}$. In this paper we are interested in 
the particular case where $\mathfrak{g}=\mathfrak{s o}(n) \oplus \mathfrak{s o}(3,2)$ and $\mathfrak{h}$ has dimension $\left(\begin{array}{l}n \\ 2\end{array}\right)-1$ for $n>4$. Given the huge number of such subalgebras, this task seems at first to be impractical or at the very least, very tiresome. Luckily, the fact that $\mathfrak{g}$ is semisimple, allows us to exploit a wonderful theorem by Nadine Kowalsky [11], generalised by Deffaf, Melnick and Zeghib [12], which characterises those homogeneous lorentzian manifolds of semisimple Lie groups. Such lorentzian manifolds come in two flavours: either the action is proper, in which case $\mathfrak{h}$ is the Lie algebra of a compact group, or else the manifold is locally isometric to the product of (anti) de Sitter space with a riemannian homogeneous manifold. In either case, we can essentially restrict to compact subalgebras $\mathfrak{h}$, which are much better known, not to mention much fewer in number.

We therefore set ourselves two tasks in this paper. The first is the classification (up to local isometry) of homogeneous backgrounds with an effective and locally transitive action of $\mathfrak{g}=\mathfrak{s o}(n) \oplus \mathfrak{s o}(3,2)$ for $n>4$, where the geometry is not of the form AdS $\times X$. To this end we will first determine the compact Lie subalgebras of $\mathfrak{g}$ (of the right dimension), up to the action of automorphisms. Lie subalgebras can be found by iterating the simpler problem of finding maximal compact subalgebras. Since $\mathfrak{g}$ is a product, this requires the Lie algebra version of Goursat's Lemma characterising the subgroups of a direct product of groups, which curiously plays such a crucial rôle in the results of $[8,9]$. The second task is the classification (again up to local isometry) of homogeneous (anti) de Sitter backgrounds with a locally transitive action of $\mathfrak{s o}(n) \oplus \mathfrak{s o}(3,2)$ for $n>4$, but which are not of Freund-Rubin type; that is, where the flux is not just equal to the volume form of a four-dimensional factor in the geometry.

The paper is organised as follows. In section 2 we review the geometry of homogeneous lorentzian manifolds. In section 2.1 we settle the notation and discuss the basics of homogeneous geometry, specialising at the end on the lorentzian case and review briefly the results of Kowalsky and of Deffaf, Melnick and Zeghib. In section 2.2 we record the necessary formulae to perform geometric computations on homogeneous lorentzian manifolds. In section 3 we prove a Goursat-type lemma for Lie algebras, characterising the Lie subalgebras of a direct product of two Lie algebras in terms of fibered products of Lie subalgebras of each of them. In section 4 we record the Lie subalgebras of $\mathfrak{s o}(n)$ for low values of $n$. This is well-known material (covered, for example, in [13]) but our purpose here is to have concrete formulae for the generators of the subalgebras in terms of the standard basis of $\mathfrak{s o}(n)$. In section 5 we write down the field equations for $d=11$ supergravity in a homogeneous Ansatz, which become a system of algebraic equations in the parameters for the metric and the 4-form. This allows us to describe our methodology in some detail in section 5.3. Section 6 contains our solution of the first of the above two tasks: the determination of homogeneous backgrounds of $\mathrm{SO}(n) \times \mathrm{SO}(3,2)$ for $n>4$, which are not of anti-de Sitter type. We will show that there are no (new) $n>5$ backgrounds, but we will exhibit a number of new (at least to us) backgrounds for $n=5$, at least one of which is supersymmetric, albeit with only $N=2$. We will explore its geometry in more detail in section 8.2.1, where we show that it is a Freund-Rubin background with underlying geometry $S^{4} \times P^{7}$, where $P$ is seven-dimensional, lorentzian Sasaki-Einstein. We also show that the background can be obtained by a "Wick rotation" from a known homogeneous 
$\mathrm{AdS}_{4}$ Freund-Rubin background. In section 7 we tackle the second of the two tasks above, namely: the determination of $\mathrm{AdS}_{4}$ backgrounds which are not of Freund-Rubin type. In section 7.1 we show that there are no de Sitter backgrounds, and we exhibit a number of new (to us) backgrounds for $n=5$ in addition to recovering some well-known backgrounds with $n=7$ (Englert), $n=6$ (Pope-Warner) and $n=5$ (Castellani-Romans-Warner). (We leave a small gap in that there is one case we have been unable to fully analyse.) Finally, in section 8 we discuss the geometry of some of the $n=5$ backgrounds found above: some of the backgrounds can only be approximated numerically, and we will have little else to say about them beyond their existence. In particular, using the method described in appendix A, we determine the actual isometry group of the backgrounds, which in some cases is slightly larger than $\mathrm{SO}(n) \times \mathrm{SO}(3,2)$. The paper ends with an appendix on the determination of the full isometry algebra of a homogeneous riemannian manifold.

\section{Homogeneous lorentzian manifolds}

In this section we review the basic notions concerning homogeneous spaces and the useful formulae for reducing their differential geometry to Lie algebraic data.

\subsection{Basic notions about homogeneous spaces}

A lorentzian manifold $(M, g)$ is homogeneous if it admits a transitive action of some Lie group by isometries. In other words, $(M, g)$ is homogeneous if there is a Lie group $G$ acting on $M$ smoothly, preserving the metric and such that any two points of $M$ are related by some element of $G$.

Let us unpack this definition. First of all, we have an action of $G$ on $M$. This is a smooth map $\alpha: G \times M \rightarrow M$, which we will denote simply by $(x, p) \mapsto x \cdot p$, such that for all $x_{i} \in G$ and $p \in M, x_{1} \cdot\left(x_{2} \cdot p\right)=\left(x_{1} x_{2}\right) \cdot p$ and $e \cdot p=p$, where $e$ denotes the identity element of $G$. The action is transitive if for some (and hence all) $p \in M$, the map $\alpha_{p}: G \rightarrow M$, defined by $\alpha_{p}(x)=x \cdot p$ is surjective. The group acts by isometries if the diffeomorphisms $\alpha_{x}: M \rightarrow M$, defined by $\alpha_{x}(p)=x \cdot p$, preserve the metric; that is, $\alpha_{x}^{*} g=g$ for all $x \in G$.

In a homogeneous space, every point is equivalent to any other point. Let us choose a point $o \in M$ and let us think of it as the origin of $M$. Let $H_{o}$ denote the subgroup of $G$ which fixes the point $o$. Since $H_{o}=\alpha_{o}^{-1}(\{o\})$ is the inverse image of a point under a continuous map, it is a closed subgroup of $G$. We call $H_{o}$ the stabiliser (subgroup) of $o$. Then $M$ is diffeomorphic to the space $G / H_{o}$ of right $H_{o}$-cosets in $G$. The point $o \in M$ corresponds to the identity coset, whereas the point $x \cdot o$ corresponds to the coset $x H_{o}$, since any one of the group elements in the coset $x H_{o}$ takes $o$ to $x \cdot o$. The differential $\left(\alpha_{x}\right)_{*}$ defines a family of linear maps $T_{o} M \rightarrow T_{x \cdot o} M$. If $x \in H_{o}$, then the differential at $o$ is an invertible linear transformation of $T_{o} M$. This is called the isotropy representation of $H_{o}$ on $T_{o} M$.

The metric $g$ defines a lorentzian inner product $g_{o}$ on each tangent space $T_{o} M$. The condition $\alpha_{x}^{*} g=g$ becomes that for all $o \in M, \alpha_{x}^{*} g_{x \cdot o}=g_{o}$. In particular, if $x \in H_{o}$, $\alpha_{x}^{*} g_{o}=g_{o}$, whence the isotropy representation of $H_{o}$ is orthogonal with respect to $g_{o}$. 
Let $\mathfrak{g}$ denote the Lie algebra of $G$, whose underlying vector space we take to be the tangent space $T_{e} G$ at the identity in $G$. Let $\mathfrak{h}_{o}$ denote the Lie subalgebra of $G$ corresponding to the stabiliser subgroup $H_{o}$ of $o$. The differential at $e \in G$ of the map $\alpha_{o}: G \rightarrow M$ is a linear map $\mathfrak{g} \rightarrow T_{o} M$ which is surjective because the action is transitive and has kernel precisely $\mathfrak{h}_{o}$. In other words, we have an exact sequence

$$
0 \longrightarrow \mathfrak{h}_{o} \longrightarrow \mathfrak{g} \stackrel{\left(\alpha_{o}\right)_{*}}{\longrightarrow} T_{o} M \longrightarrow 0
$$

not just of vector spaces, but in fact of $\mathfrak{h}_{o}$-modules. Indeed, $\mathfrak{h}_{o}$ acts on $\mathfrak{g}$ by restricting the adjoint representation of $\mathfrak{g}$ to $\mathfrak{h}_{o}$, and $\mathfrak{h}_{o}$ is a submodule precisely because $\mathfrak{h}_{o}$ is a Lie subalgebra. Then $T_{o} M$ is isomorphic as an $\mathfrak{h}_{o}$-module to $\mathfrak{g} / \mathfrak{h}_{o}$. This representation is none other than the linearisation of the isotropy representation of $H_{o}$ on $T_{o} M$. Let us prove this.

Let $h(t)$ be a regular curve in $H_{o}$ such that $h(0)=e$. Then $\alpha_{h(t)}: T_{o} M \rightarrow T_{o} M$ and the action of $h^{\prime}(0) \in \mathfrak{h}_{o}$ on $T_{o} M$ is obtained by differentiating at $t=0$. Indeed, let $v \in T_{o} M$ and choose any regular curve $\gamma(s)$ on $M$ with $\gamma(0)=o$ and $\gamma^{\prime}(0)=v$. Then

$$
\begin{aligned}
h^{\prime}(0) \cdot v & =\left.\frac{d}{d t}\right|_{t=0}\left(\alpha_{h(t)}\right)_{*} v \\
& =\left.\left.\frac{d}{d t}\right|_{t=0} \frac{d}{d s}\right|_{s=0} \alpha_{h(t)}(\gamma(s)) \\
& =\left.\left.\frac{d}{d t}\right|_{t=0} \frac{d}{d s}\right|_{s=0} h(t) \cdot \gamma(s) .
\end{aligned}
$$

Now let $g(s)$ be a regular curve in $G$ with $g(s) \cdot o=\gamma(s)$ and $g(0)=e$. Then

$$
\begin{aligned}
h^{\prime}(0) \cdot v & =\left.\left.\frac{d}{d t}\right|_{t=0} \frac{d}{d s}\right|_{s=0} h(t) \cdot(g(s) \cdot o) \\
& =\left.\left.\frac{d}{d t}\right|_{t=0} \frac{d}{d s}\right|_{s=0}(h(t) g(s)) \cdot o \\
& \left.=\left.\left.\frac{d}{d t}\right|_{t=0} \frac{d}{d s}\right|_{s=0}\left(h(t) g(s) h(t)^{-1}\right)\right) \cdot o \\
& \left.=\left.\left.\frac{d}{d t}\right|_{t=0} \frac{d}{d s}\right|_{s=0} \alpha_{o}\left(h(t) g(s) h(t)^{-1}\right)\right) \\
& \left.=\left.\frac{d}{d t}\right|_{t=0}\left(\alpha_{o}\right)_{*}\left(h(t) g^{\prime}(0) h(t)^{-1}\right)\right) \\
& =\left(\alpha_{o}\right)_{*}\left(\left[h^{\prime}(0), g^{\prime}(0)\right]\right)
\end{aligned}
$$

where $\left(\alpha_{o}\right)_{*} g^{\prime}(0)=v$. Choosing a different curve $\tilde{g}(s)$ with $\tilde{g}(s) \cdot o=g(s) \cdot o$, then $\tilde{h}(s)=$ $g(s)^{-1} g(s)$ is a curve in $H_{o}$ with $\tilde{h}(0)=e$. This means $\tilde{g}(s)=g(s) \tilde{h}(s)$, whence $\tilde{g}^{\prime}(0)=$ $g^{\prime}(0)+\tilde{h}^{\prime}(0)$, but $\tilde{h}^{\prime}(0) \in \mathfrak{h}_{o}$ and hence $\left(\alpha_{o}\right)_{*}\left(\left[h^{\prime}(0), \tilde{h}^{\prime}(0)\right]\right)=0$, so that $h^{\prime}(0) \cdot v$ is unchanged. In other words, in order to compute the action of $X \in \mathfrak{h}_{o}$ on $v \in T_{o} M$, we choose $Y \in \mathfrak{g}$ with $\left(\alpha_{o}\right)_{*} Y=v$ and then compute $\left(\alpha_{o}\right)_{*}([X, Y])$, which is independent of the lift $Y$ of $v$.

When $H_{O}$ is connected, the isotropy representation of $H_{O}$ is determined by the above representation of $\mathfrak{h}_{o}$. In practice we will assume without loss of generality that $M$ is simply 
connected and then the exact homotopy sequence of the principal $H_{o}$-bundle $G \rightarrow M$ will imply that $H_{o}$ is connected.

We can realise the linear isotropy representation explicitly by choosing a complement $\mathfrak{m}$ of $\mathfrak{h}_{o}$ in $\mathfrak{g}$, so that $\mathfrak{g}=\mathfrak{h}_{o} \oplus \mathfrak{m}$, and defining the action of $X \in \mathfrak{h}_{o}$ on $Y \in \mathfrak{m}$ by

$$
X \cdot Y=[X, Y]_{\mathfrak{m}},
$$

where, here and in the following, the subscript $\mathfrak{m}$ indicates the projection onto $\mathfrak{m}$ along $\mathfrak{h}_{o}$; that is, we simply discard the $\mathfrak{h}_{o}$-component of $[X, Y]$. If $\mathfrak{m}$ is stable under $\operatorname{ad}\left(\mathfrak{h}_{o}\right)$, so that the projection is superfluous, we say that $\mathfrak{g}=\mathfrak{h}_{o} \oplus \mathfrak{m}$ is a reductive split, and the pair $\left(\mathfrak{g}, \mathfrak{h}_{o}\right)$ is said to be reductive. This is equivalent to the splitting (in the sense of homological algebra) of the exact sequence (2.1) in the category of $\mathfrak{h}_{o}$-modules. In this case, one often says that $(M, g)$ is reductive; although this is an abuse of notation in that reductivity is not an intrinsic property of the homogeneous space, but of its description as an orbit of $G$. Not all lorentzian homogeneous manifolds need admit a reductive description; although it is known to be the case in dimension $\leq 4$ as a consequence of the classifications $[14,15]$.

Different points of $M$ can have different stabilisers, but these are conjugate in $G$, hence in particular they are isomorphic. This is why one often abbreviates homogeneous spaces as $G / H$, where $H$ denotes one of the $H_{o}$ subgroups of $G$. Let $\mathfrak{g}$ denote the Lie algebra of $G$ and let $\mathfrak{h}$ denote the Lie subalgebra corresponding to the subgroup $H$. Then a lorentzian homogeneous manifold is described locally by a pair $(\mathfrak{g}, \mathfrak{h})$ and an $\mathfrak{h}$-invariant lorentzian inner product on $\mathfrak{g} / \mathfrak{h}$, with the proviso that $\mathfrak{h}$ is the Lie algebra of a closed subgroup of $G$.

We are interested in classifying (simply-connected) eleven-dimensional homogeneous lorentzian manifolds with a transitive action of the universal covering group $G$ of $\operatorname{SO}(n) \times$ $\mathrm{SO}(3,2)$ for $n>4$. The above discussion might suggest the problem of classifying those Lie subalgebras $\mathfrak{h}$ of $\mathfrak{g}=\mathfrak{s o}(n) \oplus \mathfrak{s o}(3,2)$ of the right dimension: namely, $\operatorname{dim} \mathfrak{h}=\left(\begin{array}{l}n \\ 2\end{array}\right)-1$, which are the Lie algebras of a closed subgroup $H$ of $G$. Even in the relatively low dimension we are working in, the classification of Lie subalgebras of a semisimple Lie algebra can be a daunting task (see, e.g., [16] for the low-dimensional (anti) de Sitter algebras). Luckily, since $G$ is semisimple we may appeal to results of Nadine Kowalsky [11] and Deffaf, Melnick and Zeghib [12], which reduce the task at hand considerably by allowing us to focus on Lie algebras $\mathfrak{h}$ of compact subgroups of $G$. We will highlight the main results, which we learnt from the recent paper [17] by Dmitri Alekseevsky.

Let us recall that a continuous map between topological spaces is called proper if the inverse image of a compact set is compact. If $G$ is a Lie group acting on a manifold $M$, we say that the action is proper if the map $f: G \times M \rightarrow M \times M$, defined by $f(a, x)=(a x, x)$, is proper. Given a proper action of $G$ on $M$, we notice that $f^{-1}(x, x)=\{(a, x) \mid a x=x\}=$ $H \times\{x\}$, where $H$ is the stabiliser of $x$. Since the action is proper and $\{(x, x)\}$ is a compact set, so is $H$. Now suppose that $G$ acts properly and transitively on $M$, so that $M \cong G / H$ with $H$ compact. Then by averaging over $H$, we can assume that the linear isotropy representation of $H$ on $\mathfrak{m}$ leaves invariant a positive-definite inner product. In particular, $M=G / H$ is a reductive homogeneous space. It is proved in ([17], Prop. 4) that $M$ admits a $G$-invariant lorentzian metric if and only if the linear isotropy representation of $H$ leaves a line $\ell \subset \mathfrak{m}$ invariant. Then letting $h$ denote the positive-definite inner product on $\mathfrak{m}$ and 
$\alpha \in \mathfrak{m}^{*}$ such that ker $\alpha=\ell^{\perp}$, where $\ell^{\perp}$ is the $h$-perpendicular complement of $\ell$ in $\mathfrak{m}$, the $G$-invariant lorentzian metrics on $M$ are obtained from the inner products

$$
h-\lambda \alpha \otimes \alpha,
$$

which are lorentzian for $\lambda \gg 0$.

What about if the action of $G$ on $M$ is not proper? It is a remarkable result [11] of Nadine Kowalsky's that if a simple Lie group $G$ acts transitively by isometries on a lorentzian manifold $M$ in such a way that the action is not proper, then $M$ is locally isometric to (anti) de Sitter spacetime. Deffaf, Melnick and Zeghib [12] extended this result to the case of $G$ semisimple, with the conclusion that $M$ is now locally isometric to the product of (anti) de Sitter spacetime with a riemannian homogeneous space. Notice that in either case, we can always describe $M$ as a reductive homogeneous space.

These results will allow us to consider either $\mathrm{AdS}_{d} \times M^{11-d}$ backgrounds (one can show that there are no de Sitter backgrounds) or else restrict ourselves to the case of compact $H$. Supersymmetric Freund-Rubin backgrounds with $N \geq 4$ of the form AdS $\times M$ have been classified — see [8] for the smooth case and [9] for orbifolds — but we still need to investigate more general anti de Sitter backgrounds with flux along the internal manifold $M$. This problem was studied in the early Kaluza-Klein supergravity literature, albeit not exhaustively (see, e.g., [18] and references therein, for the progress on this problem circa 1985). We will re-examine such backgrounds, recover the known ones and exhibit ones which to our knowledge are new. Concerning the latter class of backgrounds, those with compact $H$, we must in principle distinguish between two cases: when $\mathrm{SO}(3,2)$ acts effectively and when it acts trivially; although the latter case is of dubious relevance to the AdS/CFT correspondence and will be ignored in this paper. In the former case we must look for compact Lie subalgebras $\mathfrak{h}$ of $\mathfrak{s o}(n) \oplus \mathfrak{s o}(3) \oplus \mathfrak{s o}(2)$, which is the maximally compact subalgebra of $\mathfrak{g}$, whereas in the latter case we must look for Lie subalgebras $\mathfrak{k}$ of $\mathfrak{s o}(n)$, with then $\mathfrak{h}=\mathfrak{k} \oplus \mathfrak{s o}(3,2)$. We will classify all such Lie subalgebras admitting an $\mathfrak{h}$-invariant lorentzian inner product on $\mathfrak{m}$. Since $\mathfrak{g}$ is a direct product, this will require us to learn how to determine the Lie subalgebras of a direct product of Lie algebras. This will be explained in section 3 , but not before collecting some useful formulae to do calculations in lorentzian homogeneous spaces.

\subsection{Computations in homogeneous spaces}

The purpose of this section, which overlaps with ([19], §2.3) somewhat, is to record some useful formulae for doing calculations in reductive homogeneous spaces in terms of Lie algebraic data. For more details one can consult, for example, the book [20].

Let $M=G / H$ be a reductive homogeneous space with $H$ a closed connected subgroup of $G$ and let $\mathfrak{g}=\mathfrak{h} \oplus \mathfrak{m}$ be a reductive split. The isotropy representation of $\mathfrak{h}$ on $\mathfrak{m}$ is the restriction of the adjoint action: $X \cdot Y=[X, Y]$, for $X \in \mathfrak{h}$ and $Y \in \mathfrak{m}$. Let $\langle-,-\rangle$ denote an inner product on $\mathfrak{m}$ which is invariant under the isotropy representation; that is, for all $X, Y \in \mathfrak{m}$ and $Z \in \mathfrak{h}$,

$$
\langle[Z, X], Y\rangle+\langle X,[Z, Y]\rangle
$$

This defines a $G$-invariant metric on $M$. 
More generally, there is a one-to-one correspondence between $\mathfrak{h}$-invariant tensors on $\mathfrak{m}$ and $G$-invariant tensor fields on $M$. If $F$ is a $G$-invariant tensor field on $M$, its evaluation at $o$ together with the identification of $T_{o} M$ with $\mathfrak{m}$ defines a tensor $F_{o}$ on $\mathfrak{m}$. Since $F$ is $G$-invariant, its Lie derivative at $o$ along any Killing vector vanishes. Now let $X$ be a Killing vector coming from $\mathfrak{h}$. Since its value at $o$ vanishes, the Lie derivative along $X$ is the action of the corresponding element of $\mathfrak{h}$ under the linear isotropy representation. Therefore $F_{o}$ is $\mathfrak{h}$-invariant. Conversely, let $F_{o}$ be an $\mathfrak{h}$-invariant tensor on $\mathfrak{m}$. We define a tensor field $F$ on $M$ by the condition $F(x)=a \cdot F_{o}$, where $a \in G$ is such that $a \cdot o=x$, which exists since $G$ acts transitively. This is actually well defined because $F_{0}$ is $H$-invariant. Indeed, let $b \in G$ be such that $b \cdot o=x$. Then $b^{-1} a \cdot o=o$, whence $b^{-1} a \in H$. Therefore $b \cdot F_{o}=b \cdot b^{-1} a \cdot F_{o}=a \cdot F_{o}$. The tensor field $F$ so defined is clearly $G$-invariant, since for all $a \in G$ and $x \in M, F(a \cdot x)=a \cdot F(x)$, since both sides equal $a b \cdot F_{o}$, where $b \in G$ is any element such that $b \cdot o=x$.

Let $X, Y, Z$ be Killing vectors on $M=G / H$. The Koszul formula for the Levi-Civita connection reads

$$
2 g\left(\nabla_{X} Y, Z\right)=g([X, Y], Z)+g([X, Z], Y)+g(X,[Y, Z]) .
$$

At the identity coset $o \in M$ and assuming that $X, Y, Z$ are Killing vectors in $\mathfrak{m}$, the chosen complement of $\mathfrak{h}$ in $\mathfrak{g}$, then

$$
\left.\nabla_{X} Y\right|_{o}=-\frac{1}{2}[X, Y]_{\mathfrak{m}}+U(X, Y)
$$

where $U: \mathfrak{m} \times \mathfrak{m} \rightarrow \mathfrak{m}$ is a symmetric tensor given by ${ }^{1}$

$$
2\langle U(X, Y), Z\rangle=\left\langle[Z, X]_{\mathfrak{m}}, Y\right\rangle+\left\langle[Z, Y]_{\mathfrak{m}}, X\right\rangle
$$

for all $Z \in \mathfrak{m}$ and where the subscript denotes the projection of $[Z, X] \in \mathfrak{g}$ to $\mathfrak{m}$. It should be remarked that (2.8) is only valid at $o \in M$, since $\nabla_{X} Y$ is not generally a Killing vector. Of course, since $\nabla$ is G-invariant, then one can determine $\left.\nabla_{X} Y\right|_{p}$ at any other point by acting with any isometry relating $o$ and $p$.

For a reductive homogeneous space, the $U$-tensor is invariant under the linear isotropy representation. The vanishing of the $U$-tensor characterises the class of homogeneous spaces known as naturally reductive. In those spaces, the geodesics of the invariant connection and the Levi-Civita connection agree.

The Riemann curvature tensor is $G$-invariant and it can be computed at $o$. One obtains, for $X, Y, Z, W$ vectors in $\mathfrak{m}$, the curvature tensor at $o$ is given by

$$
\begin{aligned}
R(X, Y, Z, W)= & \langle U(X, W), U(Y, Z)\rangle-\langle U(X, Z), U(Y, W)\rangle \\
& +\frac{1}{12}\left\langle[X,[Y, Z]]_{\mathfrak{m}}, W\right\rangle-\frac{1}{12}\left\langle[X,[Y, W]]_{\mathfrak{m}}, Z\right\rangle \\
& -\frac{1}{6}\left\langle[X,[Z, W]]_{\mathfrak{m}}, Y\right\rangle-\frac{1}{12}\left\langle[Y,[X, Z]]_{\mathfrak{m}}, W\right\rangle
\end{aligned}
$$

\footnotetext{
${ }^{1}$ The apparent difference in sign between equation (2.7) and equations (2.8) and (2.9) stems from the fact that Killing vectors on $G / H$ generate left translations on $G$, whence they are right-invariant. Thus the map $\mathfrak{g} \rightarrow$ Killing vector fields is an anti-homomorphism.
} 


$$
\begin{aligned}
& +\frac{1}{12}\left\langle[Y,[X, W]]_{\mathfrak{m}}, Z\right\rangle+\frac{1}{6}\left\langle[Y,[Z, W]]_{\mathfrak{m}}, X\right\rangle \\
& -\frac{1}{6}\left\langle[Z,[X, Y]]_{\mathfrak{m}}, W\right\rangle-\frac{1}{12}\left\langle[Z,[X, W]]_{\mathfrak{m}}, Y\right\rangle \\
& +\frac{1}{12}\left\langle[Z,[Y, W]]_{\mathfrak{m}}, X\right\rangle+\frac{1}{6}\left\langle[W,[X, Y]]_{\mathfrak{m}}, Z\right\rangle \\
& +\frac{1}{12}\left\langle[W,[X, Z]]_{\mathfrak{m}}, Y\right\rangle-\frac{1}{12}\left\langle[W,[Y, Z]]_{\mathfrak{m}}, X\right\rangle \\
& -\frac{1}{2}\left\langle[X, Y]_{\mathfrak{m}},[Z, W]_{\mathfrak{m}}\right\rangle-\frac{1}{4}\left\langle[X, Z]_{\mathfrak{m}},[Y, W]_{\mathfrak{m}}\right\rangle+\frac{1}{4}\left\langle[X, W]_{\mathfrak{m}},[Y, Z]_{\mathfrak{m}}\right\rangle
\end{aligned}
$$

which can be obtained by polarisation from the simpler expression for $K(X, Y):=$ $\langle R(X, Y) X, Y\rangle$, which is also easier to derive. Indeed, and for completeness, one has

$$
\begin{aligned}
6 R(X, Y, Z, W)= & K(X+Z, Y+W)-K(Y+Z, X+W) \\
& -K(Y+W, X)+K(Y+Z, X)-K(X+Z, Y)+K(X+W, Y) \\
& -K(Y+W, Z)+K(X+W, Z)-K(X+Z, W)+K(Y+Z, W) \\
& +K(X, W)-K(X, W)-K(Y, W)+K(Y, Z)-K(X, Z),
\end{aligned}
$$

where

$$
\begin{aligned}
K(X, Y)= & -\frac{3}{4}\left|[X, Y]_{\mathfrak{m}}\right|^{2}-\frac{1}{2}\left\langle[X,[X, Y]]_{\mathfrak{m}}, Y\right\rangle-\frac{1}{2}\left\langle[Y,[Y, X]]_{\mathfrak{m}}, X\right\rangle+|U(X, Y)|^{2} \\
& -\langle U(X, X), U(Y, Y)\rangle
\end{aligned}
$$

and where $|-|^{2}$ is the (indefinite) norm associated to $\langle-,-\rangle$.

Similarly, we can obtain the Ricci tensor by polarisation from

$$
\begin{aligned}
\operatorname{Ric}(X, X)= & -\frac{1}{2} \sum_{i}\left\langle\left[X, X_{i}\right]_{\mathfrak{m}},\left[X, X^{i}\right]_{\mathfrak{m}}\right\rangle-\frac{1}{2} \sum_{i}\left\langle\left[X,\left[X, X_{i}\right]_{\mathfrak{m}}\right]_{\mathfrak{m}}, X^{i}\right\rangle-\sum_{i}\left\langle\left[X,\left[X, X_{i}\right]_{\mathfrak{h}}\right], X^{i}\right\rangle \\
& -\sum_{i}\left\langle\left[U\left(X_{i}, X^{i}\right), X\right]_{\mathfrak{m}}, X\right\rangle+\frac{1}{4} \sum_{i, j}\left\langle\left[X_{i}, X_{j}\right]_{\mathfrak{m}}, X\right\rangle\left\langle\left[X^{i}, X^{j}\right]_{\mathfrak{m}}, X\right\rangle
\end{aligned}
$$

where $X_{i}$ is a pseudo-orthonormal basis with $\left\langle X_{i}, X^{j}\right\rangle=\delta_{i}^{j}$. The Ricci scalar is given by $R=\sum_{i} \operatorname{Ric}\left(X_{i}, X^{i}\right)$.

It is convenient to write down the expression for the Ricci tensor in terms of a local frame, since this is what is used in computations. So let $Y_{i}$ denote a basis for $\mathfrak{m}$ with $\left\langle Y_{i}, Y_{j}\right\rangle=g_{i j}$ and let $X_{a}$ denote a basis for $\mathfrak{h}$. The structure constants are $\left[X_{a}, Y_{i}\right]=f_{a i}{ }^{j} Y_{j}$ (assumed reductive) and $\left[Y_{i}, Y_{j}\right]=f_{i j}{ }^{k} Y_{k}+f_{i j}{ }^{a} X_{a}$. We can raise and lower $\mathfrak{m}$-indices using $g$. In this notation, we find that the Ricci tensor is given by:

$$
R_{i j}=-\frac{1}{2} f_{i}{ }^{k \ell} f_{j k \ell}-\frac{1}{2} f_{i k}{ }^{\ell} f_{j \ell}{ }^{k}+\frac{1}{2} f_{i k}{ }^{a} f_{a j}{ }^{k}+\frac{1}{2} f_{j k}{ }^{a} f_{a i}{ }^{k}-\frac{1}{2} f_{k \ell}{ }^{\ell} f^{k}{ }_{i j}-\frac{1}{2} f_{k \ell}{ }^{\ell} f_{j i}^{k}+\frac{1}{4} f_{k \ell i} f^{k \ell}{ }_{j} .
$$

Let $\Omega^{\bullet}(M)$ denote the de Rham complex on $M$ and $\Omega^{\bullet}(M)^{G}$ the subcomplex of $G$ invariant differential forms. The value at $o \in M$ of a $G$-invariant differential $k$-form $\omega$ on $M$ is an $H$-invariant element of $\Lambda^{k} \mathfrak{m}^{*}$. Its exterior derivative and its codifferential can be expressed purely in terms of the Lie algebraic data defining the homogeneous space. If $X_{i}$ are Killing vectors in $\mathfrak{m}$, then the exterior derivative of $\omega$ is given by

$$
d \omega\left(X_{1}, \ldots, X_{k+1}\right)=\sum_{1 \leq i<j \leq k+1}(-1)^{i+j} \omega\left(\left[X_{i}, X_{j}\right]_{\mathfrak{m}}, X_{1}, \ldots, \widehat{X}_{i}, \ldots, \widehat{X}_{j}, \ldots, X_{k+1}\right)
$$


where a hat adorning a symbol denotes its omission. Perhaps the simplest proof of this statement is to localise the complex $\Omega^{\bullet}(M)^{G}$ as the subcomplex of left-invariant differential forms on $G$ which are basic. In other words, we view $M$ as the base of a principal $H$-bundle with total space $G$. A $G$-invariant differential form $\omega$ on $M$ pulls back to a left-invariant form on $G$ whose value $\omega_{e}$ at the identity is both horizontal: $\imath_{X} \omega_{e}=0$ for all $X \in \mathfrak{h}$, and invariant under the adjoint action of $\mathfrak{h}$. We then use the standard formulae (see, e.g., [21]) for the differential of a left-invariant form on $G$, after checking that the basic forms indeed form a subcomplex.

In computations, a more convenient way to compute the exterior derivative of an invariant form is the following. Let $\left(Y_{i}\right)$ be a basis for $\mathfrak{m}$ such that $\left[Y_{i}, Y_{j}\right]_{\mathfrak{m}}=\sum_{k} f_{i j}{ }^{k} Y_{k}$. Then let $\left(\theta^{i}\right)$ be the canonically dual basis for $\mathfrak{m}^{*}$. Then it follows from equation $(2.15)$ that

$$
d \theta^{k}=-\frac{1}{2} \sum_{i, j} f_{i j}^{k} \theta^{i} \wedge \theta^{j}
$$

We then extend $d$ as a derivation to a general invariant form. Therefore, if $F$ is an invariant 4-form, so that $F=\frac{1}{4 !} F_{i j k l} \theta^{i j k l}$ (with the Einstein summation convention in force), then

$$
d F=-\frac{1}{12} f_{m n}^{i} F_{i j k l} \theta^{j k l m n},
$$

or explicitly,

$$
\begin{aligned}
(d F)_{j k l m n}= & f_{j k}{ }^{i} F_{i l m n}-f_{j l}{ }^{i} F_{i k m n}+f_{j m}{ }^{i} F_{i k l n}-f_{j n}{ }^{i} F_{i k l m}+f_{k l}{ }^{i} F_{i j m n} \\
& -f_{k m}{ }^{i} F_{i j l n}+f_{k n}{ }^{i} F_{i j l m}+f_{l m}{ }^{i} F_{i j k n}-f_{l n}{ }^{i} F_{i j k m}+f_{m n}{ }^{i} F_{i j k l}
\end{aligned}
$$

To describe the codifferential, let us introduce dual bases $\left(Y_{i}\right)$ and $\left(Y^{i}\right)$ for $\mathfrak{m}$ such that $\left\langle Y_{i}, Y^{j}\right\rangle=\delta_{i}^{j}$. Then we have

$$
\begin{aligned}
\delta \omega\left(X_{1}, \ldots, X_{k-1}\right)= & \sum_{i=1}^{\operatorname{dim}} \sum_{j=1}^{M-1} \omega\left(Y^{i}, X_{1}, \ldots,-\frac{1}{2}\left[Y_{i}, X_{j}\right]_{\mathfrak{m}}-U\left(Y_{i}, X_{j}\right), \ldots, X_{k-1}\right) \\
& -\sum_{i=1}^{\operatorname{dim} M} \omega\left(U\left(Y^{i}, Y_{i}\right), X_{1}, \ldots, X_{k-1}\right) .
\end{aligned}
$$

We can write this in terms of a frame $Y_{i}$ for $\mathfrak{m}$, which is perhaps more useful in computations. For $F$ an invariant 4 -form, we have

$$
\begin{aligned}
(\delta F)_{i j k}= & -\left(\frac{1}{2} f_{m i}{ }^{n}+U_{m i}{ }^{n}\right) F^{m}{ }_{n j k}-\left(\frac{1}{2} f_{m j}{ }^{n}+U_{m j}{ }^{n}\right) F^{m}{ }_{i n k} \\
& -\left(\frac{1}{2} f_{m k}{ }^{n}+U_{m k}{ }^{n}\right) F^{m}{ }_{i j n}-U_{m}{ }^{m n} F_{n i j k} .
\end{aligned}
$$

\section{Lie subalgebras of a direct product}

In this section we prove a result characterising Lie subalgebras of the direct product of two Lie algebras. This result is necessary for the determination of the Lie subalgebras of $\mathfrak{s o}(n) \oplus \mathfrak{s o}(3,2)$. It is by no means original, but we know of no good reference. 
Let $\mathfrak{g}_{L}$ and $\mathfrak{g}_{R}$ be two real Lie algebras and let $\mathfrak{g}=\mathfrak{g}_{L} \oplus \mathfrak{g}_{R}$ be their product. Elements of $\mathfrak{g}$ are pairs $\left(X_{L}, X_{R}\right)$ with $X_{L} \in \mathfrak{g}_{L}$ and $X_{R} \in \mathfrak{g}_{R}$. The Lie bracket in $\mathfrak{g}$ of two such elements $\left(X_{L}, X_{R}\right)$ and $\left(Y_{L}, Y_{R}\right)$ is given by the pair $\left(\left[X_{L}, Y_{L}\right],\left[X_{R}, Y_{R}\right]\right)$.

We are interested in Lie subalgebras $\mathfrak{h}$ of $\mathfrak{g}$. This is analogous to the determination of subgroups of a product group, which is solved by Goursat's Lemma [22]. As a result we will also call this the Goursat Lemma for Lie algebras.

Let $\pi_{L}: \mathfrak{g} \rightarrow \mathfrak{g}_{L}$ and $\pi_{R}: \mathfrak{g} \rightarrow \mathfrak{g}_{R}$ denote the projections onto each factor: they are Lie algebra homomorphisms. Let $\mathfrak{h}_{L}$ and $\mathfrak{h}_{R}$ denote, respectively, the image of the subalgebra $\mathfrak{h}$ under $\pi_{L}$ and $\pi_{R}$. They are Lie subalgebras of $\mathfrak{g}_{L}$ and $\mathfrak{g}_{R}$, respectively. Let us define $\mathfrak{h}_{L}^{0}:=\pi_{L}\left(\operatorname{ker} \pi_{R} \cap \mathfrak{h}\right)$ and $\mathfrak{h}_{R}^{0}:=\pi_{R}\left(\operatorname{ker} \pi_{L} \cap \mathfrak{h}\right)$. One checks that they are ideals of $\mathfrak{h}_{L}$ and $\mathfrak{h}_{R}$, respectively. This means that on $\mathfrak{h}_{L} / \mathfrak{h}_{L}^{0}$ and $\mathfrak{h}_{R} / \mathfrak{h}_{R}^{0}$ we can define Lie algebra structures. Goursat's Lemma says that these two Lie algebras are isomorphic. Let us understand this.

The Lie algebra $\mathfrak{h}_{L}$ consists of those $X_{L} \in \mathfrak{g}_{L}$ such that there is some $X_{R} \in \mathfrak{g}_{R}$ with $X_{L}+X_{R} \in \mathfrak{h}$, and similarly $\mathfrak{h}_{R}$ consists of those $X_{R} \in \mathfrak{g}_{R}$ such that there is some $X_{L} \in \mathfrak{g}_{L}$ with $X_{L}+X_{R} \in \mathfrak{h}$. At the same time, $\mathfrak{h}_{L}^{0}$ consists of those $X_{L} \in \mathfrak{g}_{L}$ which are also in $\mathfrak{h}$, whereas $\mathfrak{h}_{R}^{0}$ consists of those $X_{R} \in \mathfrak{g}_{R}$ which are also in $\mathfrak{h}$. Let us define a linear map $\varphi: \mathfrak{h}_{L} \rightarrow \mathfrak{h}_{R} / \mathfrak{h}_{R}^{0}$ as follows. Let $X_{L} \in \mathfrak{h}_{L}$. Then this means that there is some $X_{R} \in \mathfrak{h}_{R}$ such that $X_{L}+X_{R} \in \mathfrak{h}$. Define $\varphi\left(X_{L}\right)=X_{R} \bmod \mathfrak{h}_{R}^{0}$. This map is well defined because if both $X_{L}+X_{R}$ and $X_{L}+X_{R}^{\prime}$ are in $\mathfrak{h}$, so is their difference, whence $X_{R}-X_{R}^{\prime} \in \mathfrak{h}_{R}^{0}$. Now $\varphi$ is surjective, since for every $X_{R} \in \mathfrak{h}_{R}$, there is some $X_{L} \in \mathfrak{h}_{L}$ with $X_{L}+X_{R} \in \mathfrak{h}$, whence $\varphi\left(X_{L}\right)=X_{R} \bmod \mathfrak{h}_{R}^{0}$. Finally, the kernel of $\varphi$ consists of those $X_{L} \in \mathfrak{h}_{L}$ such that there is some $X_{R} \in \mathfrak{h}_{R}^{0}$ such that $X_{L}+X_{R} \in \mathfrak{h}$. But $X_{R} \in \mathfrak{h}$, whence $X_{L} \in \mathfrak{h}$ and hence $X_{L} \in \mathfrak{h}_{L}^{0}$. Conversely if $X_{L} \in \mathfrak{h}_{L}^{0}, X_{L} \in \mathfrak{h}$ so that $\varphi\left(X_{L}\right)=0 \bmod \mathfrak{h}_{R}^{0}$, hence $\operatorname{ker} \varphi=\mathfrak{h}_{L}^{0}$. In summary, $\varphi$ defines an isomorphism $\mathfrak{h}_{L} / \mathfrak{h}_{L}^{0} \cong \mathfrak{h}_{R} / \mathfrak{h}_{R}^{0}$.

Notice that the dimension of $\mathfrak{h}$ obeys

$$
\operatorname{dim} \mathfrak{h}=\operatorname{dim} \mathfrak{h}_{L}+\operatorname{dim} \mathfrak{h}_{R}^{0}=\operatorname{dim} \mathfrak{h}_{R}+\operatorname{dim} \mathfrak{h}_{L}^{0},
$$

as a consequence of the Euler-Poincaré principle applied to the exact sequences

$$
0 \longrightarrow \mathfrak{h}_{R}^{0} \longrightarrow \mathfrak{h} \stackrel{\pi_{L}}{\longrightarrow} \mathfrak{h}_{L} \longrightarrow 0
$$

and

$$
0 \longrightarrow \mathfrak{h}_{L}^{0} \longrightarrow \mathfrak{h} \stackrel{\pi_{R}}{\longrightarrow} \mathfrak{h}_{R} \longrightarrow 0
$$

Goursat's Lemma suggests a systematic approach to the determination of the Lie subalgebras of $\mathfrak{g}_{L} \oplus \mathfrak{g}_{R}$, which is particularly feasible when $\mathfrak{g}_{L}$ and $\mathfrak{g}_{R}$ have low dimension.

Namely, we look for Lie subalgebras $\mathfrak{h}_{L} \subset \mathfrak{g}_{L}$ and $\mathfrak{h}_{R} \subset \mathfrak{g}_{R}$ which have quotients isomorphic to $\mathfrak{q}$, say. Let $f_{L}: \mathfrak{h}_{L} \rightarrow \mathfrak{q}$ and $f_{R}: \mathfrak{h}_{R} \rightarrow \mathfrak{q}$ be the corresponding surjections. Let $\varphi \in$ Aut $\mathfrak{q}$ denote an automorphism of $\mathfrak{q}$. Then we may form the Lie subalgebra $\mathfrak{h}_{L} \oplus_{(\mathfrak{q}, \varphi)} \mathfrak{h}_{R}$ of $\mathfrak{h}_{L} \oplus \mathfrak{h}_{R}$ defined by

$$
\mathfrak{h}_{L} \oplus_{(\mathfrak{q}, \varphi)} \mathfrak{h}_{R}:=\left\{\left(X_{L}, X_{R}\right) \in \mathfrak{h}_{L} \oplus \mathfrak{h}_{R} \mid f_{L}\left(X_{L}\right)=\varphi\left(f_{R}\left(X_{R}\right)\right)\right\}
$$

Of course, we need only consider automorphisms $\varphi$ which are not induced by automorphisms of $\mathfrak{h}_{L}$ or $\mathfrak{h}_{R}$. We record here the following useful dimension formula which follows 
from equation (3.1):

$$
\operatorname{dim}\left(\mathfrak{h}_{L} \oplus_{(\mathfrak{q}, \varphi)} \mathfrak{h}_{R}\right)=\operatorname{dim} \mathfrak{h}_{L}+\operatorname{dim} \mathfrak{h}_{R}-\operatorname{dim} \mathfrak{q}
$$

A commonly occurring special case is when one of $\mathfrak{h}_{L} \rightarrow \mathfrak{q}$ or $\mathfrak{h}_{R} \rightarrow \mathfrak{q}$ is an isomorphism. For definiteness let us assume that it is $\mathfrak{h}_{R} \rightarrow \mathfrak{q}$ which is an isomorphism. Then we get a Lie algebra homomorphism $\mathfrak{h}_{L} \rightarrow \mathfrak{h}_{R}$ obtained by composing $\mathfrak{h}_{L} \rightarrow \mathfrak{q}$ with the inverse of $\mathfrak{h}_{R} \rightarrow \mathfrak{q}$. In fact, we get a family of such homomorphisms labelled by the automorphisms of $\mathfrak{q}$ or, equivalently, of $\mathfrak{h}_{R}$. The fibered product which Goursat's Lemma describes is now the graph in $\mathfrak{h}_{L} \oplus \mathfrak{h}_{R}$ of such a homomorphism $\mathfrak{h}_{L} \rightarrow \mathfrak{h}_{R}$. The resulting Lie algebra is abstractly isomorphic to $\mathfrak{h}_{L}$.

\section{Lie subalgebras of $\mathfrak{s o}(n)$}

We first consider the Lie subalgebras of $\mathfrak{s o}(n)$. We will be interested in $n \leq 7$, since the maximally supersymmetric backgrounds have been classified [23] and there are precisely two such classes of backgrounds with $\mathfrak{o s p}(8 \mid 4)$ Killing superalgebra: namely, $\operatorname{AdS}_{4} \times S^{7}$ and $\mathrm{AdS}_{4} \times S^{7} / \mathbb{Z}_{2}$. For backgrounds of the form $\mathrm{AdS}_{4} \times X^{7}$, it is known that $N>6$ implies maximal supersymmetry, but this has not been shown for more general backgrounds. Let us work our way to $n=7$.

Let us say that a Lie subalgebra is maximal if it is proper and is not properly contained in a proper Lie subalgebra. Clearly, it is enough to determine the maximal subalgebras and iterate in order to determine all the proper subalgebras. The maximal subalgebras of the simple Lie algebras we shall be interested in have been tabulated in [13] using methods introduced by Dynkin.

For us, the Lie algebra $\mathfrak{s o}(n)$ is the real span of $L_{a b}$, for $1 \leq a<b \leq n$, with Lie brackets

$$
\left[L_{a b}, L_{c d}\right]=\delta_{b c} L_{a d}-\delta_{a c} L_{b d}-\delta_{b d} L_{a c}+\delta_{a d} L_{b c} .
$$

Notice that for any $k<n$, the subspace spanned by $L_{a b}$ where we restrict $1 \leq a<b \leq k$ is a Lie subalgebra isomorphic to $\mathfrak{s o}(k)$. We will attempt to label Lie algebras in such a way that $\mathfrak{s o}(k)$ will always denote this subalgebra. Other subalgebras isomorphic to $\mathfrak{s o}(k)$ will be adorned in various ways in order to distinguish them. Hopefully this will not be too confusing.

\subsection{Lie subalgebras of $\mathfrak{s o}(2)$}

First of all, it is clear that $\mathfrak{s o}(2)=\mathbb{R}\left\langle L_{12}\right\rangle$ has no proper subalgebras.

\subsection{Lie subalgebras of $\mathfrak{s o}(3)$}

Next we consider $\mathfrak{s o}(3)=\mathbb{R}\left\langle L_{12}, L_{13}, L_{23}\right\rangle$. There is only one proper Lie subalgebra of $\mathfrak{s o}(3)$ up to equivalence and it is one-dimensional. Indeed, $\mathfrak{s o}(3)$ can be identified with $\mathbb{R}^{3}$ with the Lie bracket given by the vector cross product. Hence if a Lie subalgebra $\mathfrak{h} \subset \mathfrak{s o}(3)$ 
has dimension greater than 1 it means that there are two linearly independent vectors $x$ and $y$ in $\mathfrak{h}$, but then their cross product $x \times y$ is in $\mathfrak{h}$ but is linearly independent from $x$ and $y$, whence $\mathfrak{h}=\mathfrak{s o}(3)$. We will choose the unique (up to equivalence) Lie subalgebra of $\mathfrak{s o}(3)$ to be $\mathfrak{s o}(2)$, spanned by $L_{12}$.

\subsection{Lie subalgebras of $\mathfrak{s o}(4)$}

Unlike $\mathfrak{s o}(n)$ for all other $n \geq 3, \mathfrak{s o}(4)$ is not simple: it is isomorphic to two copies of $\mathfrak{s o}(3)$, which we will call $\mathfrak{s o}(3)_{ \pm}$since they correspond to the \pm 1 eigenspaces of the Hodge star acting on $\Lambda^{2} \mathbb{R}^{4}$ to which $\mathfrak{s o}(4)$ is isomorphic as a vector space and indeed as a representation. More precisely, let us define $L_{i}^{ \pm}:=\mp \frac{1}{2}\left(L_{i 4} \pm \frac{1}{2} \varepsilon_{i j k} L_{j k}\right)$, for $i=1,2,3$, where $\varepsilon_{123}=1$ in our conventions. In other words,

$$
L_{1}^{ \pm}=\mp \frac{1}{2}\left(L_{14} \pm L_{23}\right) \quad L_{2}^{ \pm}=\mp \frac{1}{2}\left(L_{24} \mp L_{13}\right) \quad L_{3}^{ \pm}=\mp \frac{1}{2}\left(L_{34} \pm L_{12}\right),
$$

which obey the following Lie brackets $\left[L_{i}^{ \pm}, L_{j}^{ \pm}\right]=\varepsilon_{i j k} L_{k}^{ \pm}$and $\left[L_{i}^{+}, L_{j}^{-}\right]=0$.

There are two inequivalent maximal subalgebras of $\mathfrak{s o}(4)$ : namely, $\mathfrak{s o}(3)_{+} \oplus \mathfrak{s o}(2)_{-}$, with generators $\left(L_{i}^{+}, L_{3}^{-}\right)$, for $i=1,2,3$, and the diagonal subalgebra of $\mathfrak{s o}(3)_{+} \oplus \mathfrak{s o}(3)_{-}$, with generators $\left(L_{i}^{+}+L_{i}^{-}\right)$, for $i=1,2,3$, which is thus precisely $\mathfrak{s o}(3)$ as defined above. One might expect also a subalgebra $\mathfrak{s o}(2)_{+} \oplus \mathfrak{s o}(3)_{-}$, but this is related to $\mathfrak{s o}(3)_{+} \oplus \mathfrak{s o}(2)-$ via an automorphism of $\mathfrak{s o}(4)$ : namely, $L_{i}^{ \pm} \mapsto L_{i}^{\mp}$. Geometrically it corresponds to orientation reversal in $\mathbb{R}^{4}$. The maximal subalgebras of $\mathfrak{s o}(3)$ have been determined above, so it remains to determine the maximal subalgebras of $\mathfrak{s o}(3)_{+} \oplus \mathfrak{s o}(2)_{-}$.

There are two inequivalent maximal subalgebras of $\mathfrak{s o}(3)_{+} \oplus \mathfrak{s o}(2)_{-}:$namely, $\mathfrak{s o}(3)_{+}$, spanned by $\left(L_{i}^{+}\right)$for $i=1,2,3$ and $\mathfrak{s o}(2)_{+} \oplus \mathfrak{s o}(2)_{-}$, spanned by $\left(L_{3}^{+}, L_{3}^{-}\right)$. All proper subalgebras of $\mathfrak{s o}(2)_{+} \oplus \mathfrak{s o}(2)_{-}$are one-dimensional and hence maximal. There is a pencil of such subalgebras, corresponding to the span of $\alpha L_{3}^{+}+\beta L_{3}^{-}$, for fixed $\alpha, \beta$, where the pair $(\alpha, \beta)$ is defined up to multiplication by a nonzero real number: that is, $(\alpha, \beta) \sim(\lambda \alpha, \lambda \beta)$ for some $\lambda \neq 0$. Notice that the automorphism corresponding to orientation reversal on $\mathbb{R}^{4}$ exchanges $\alpha$ and $\beta$, whence one must impose the condition $\alpha \geq \beta$, say, in order not to over-count. We can set $\alpha=1$ without loss of generality and parametrise the subalgebras by a real number $\beta \in[0,1]$. Thus we let $\mathfrak{s o}(2)_{\beta}$ denote the span of $L_{3}^{+}+\beta L_{3}^{-}$. Notice that $\mathfrak{s o}(2)_{\beta=0}=\mathfrak{s o}(2)_{+}$and $\mathfrak{s o}(2)_{\beta=1}=\mathfrak{s o}(2)$, whence the need to impose $0<\beta<1$.

At this moment we should point out a generic fact. We are interested in manifolds $G / H$, where $H$ is a closed subgroup of $G$. This condition typically translates into the rationality of the parameters defining the Lie subalgebra. For example, the Lie subalgebra $\mathfrak{s o}(2)_{\beta}$ of $\mathfrak{s o}(4)$ is the Lie algebra of a subgroup which is dense in a torus if $\beta$ is irrational, hence for it to correspond to a closed subgroup, we must impose that $\beta$ be rational.

Putting all this together we get the following Hasse diagram of nontrivial subalgebras of $\mathfrak{s o}(4)$ up to equivalence. Following an edge upwards denotes inclusion of a maximal subalgebra and subalgebras at the same height have the same dimension, as indicated in 
the left-hand column.

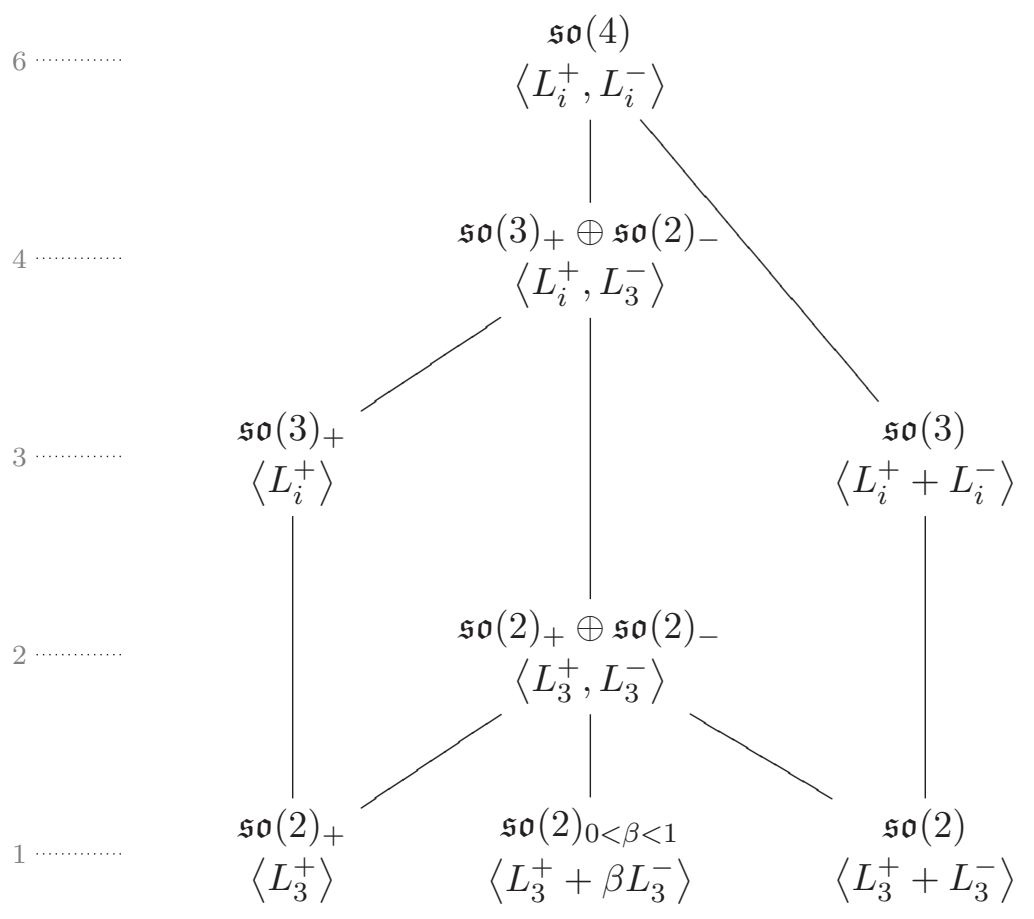

\subsection{Lie subalgebras of $\mathfrak{s o}(5)$}

The Lie algebra $\mathfrak{s o}(5)$ has three inequivalent maximal subalgebras. Two of them decompose the 5-dimensional real representation: $\mathfrak{s o}(4)$, which leaves invariant a line, and $\mathfrak{s o}(3) \oplus$ $\mathfrak{s o}(2)_{45}$, spanned by $\left(L_{12}, L_{13}, L_{23}, L_{45}\right)$. The third maximal subalgebra, isomorphic to $\mathfrak{s o}(3)$, acts irreducibly both on the vector and spinor representations. We denote it $\mathfrak{s o}(3)_{\text {irr }}$ and an explicit basis is given by

$$
\mathfrak{s o}(3)_{\text {irr }}=\mathbb{R}\left\langle L_{15}+2 L_{24}, \sqrt{3} L_{35}+L_{12}-L_{45}, \sqrt{3} L_{13}+L_{14}+L_{25}\right\rangle
$$

Any $\mathfrak{s o}(2)$ subalgebra of $\mathfrak{s o}(3)_{\text {irr }}$ leaves invariant precisely a line in $\mathbb{R}^{5}$. This means that it is contained in the maximal $\mathfrak{s o}(4)$ subalgebra. In fact, comparing characteristic polynomials of the resulting linear transformations of $\mathbb{R}^{5}$ shows that it is equivalent to an $\mathfrak{s o}(2)_{\beta=\frac{1}{3}}$ subalgebra, hence already included under the subalgebras of $\mathfrak{s o}(4)$. There are two maximal subalgebras of $\mathfrak{s o}(3) \oplus \mathfrak{s o}(2){ }_{45}$. One of them is of course $\mathfrak{s o}(3)$, whereas the other is equivalent to $\mathfrak{s o}(2)_{+} \oplus \mathfrak{s o}(2)_{-}$. This allows us to determine the Hasse diagram of nontrivial subalgebras 
of $\mathfrak{s o}(5)$ from that of $\mathfrak{s o}(4)$.

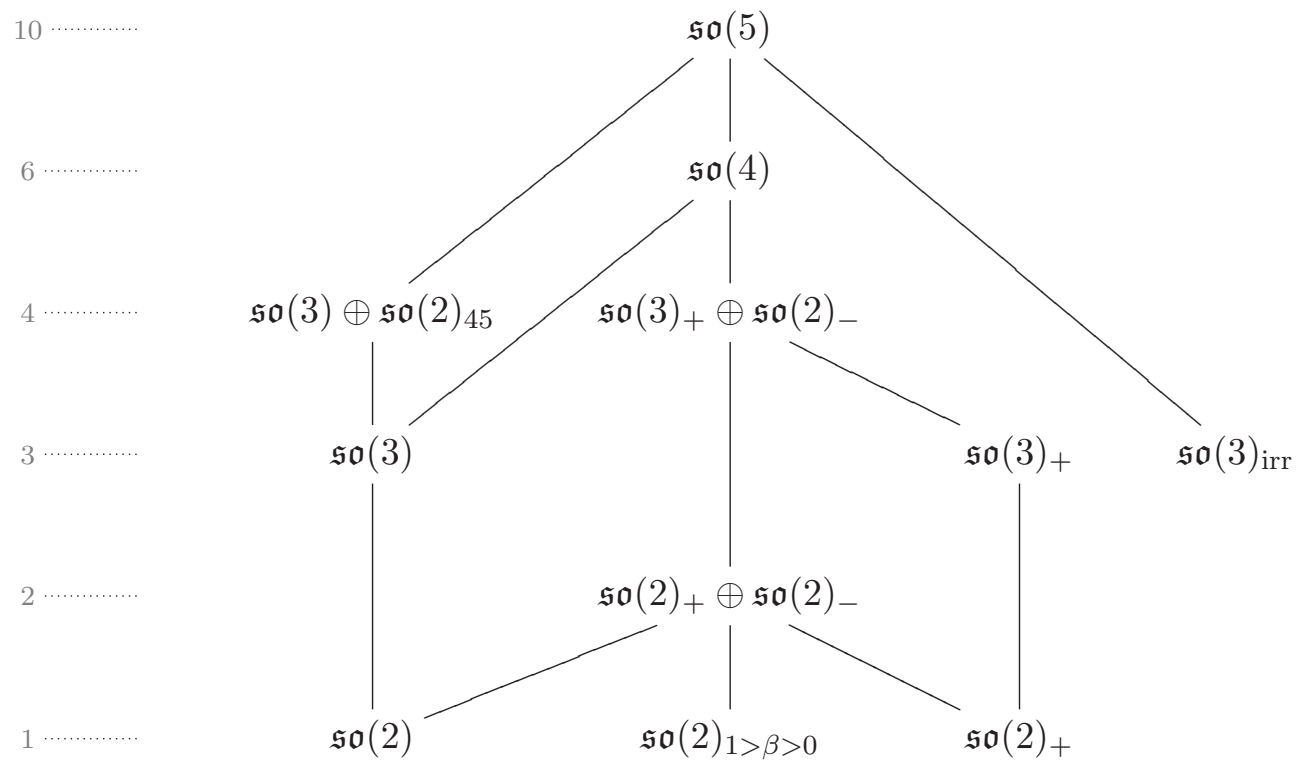

We have omitted some subalgebras of $\mathfrak{s o}(3) \oplus \mathfrak{s o}(2)_{45}$ and of $\mathfrak{s o}(3)_{\text {irr }}$ since as explained above, they are equivalent to (albeit not the same as) subalgebras already included in the diagram.

\subsection{Lie subalgebras of $\mathfrak{s o}(6)$}

The Lie algebra $\mathfrak{s o}(6)$ has four inequivalent maximal subalgebras. Three of them decompose the 6-dimensional representation: namely, $\mathfrak{s o}(5), \mathfrak{s o}(4) \oplus \mathfrak{s o}(2)_{56}, \mathfrak{s o}(3) \oplus \mathfrak{s o}(3)_{456}$; whereas one acts irreducibly on this representation: namely, $\mathfrak{u}(3) \cong \mathfrak{s u}(3) \oplus \mathfrak{u}(1)$. The top of the Hasse diagram is given below.

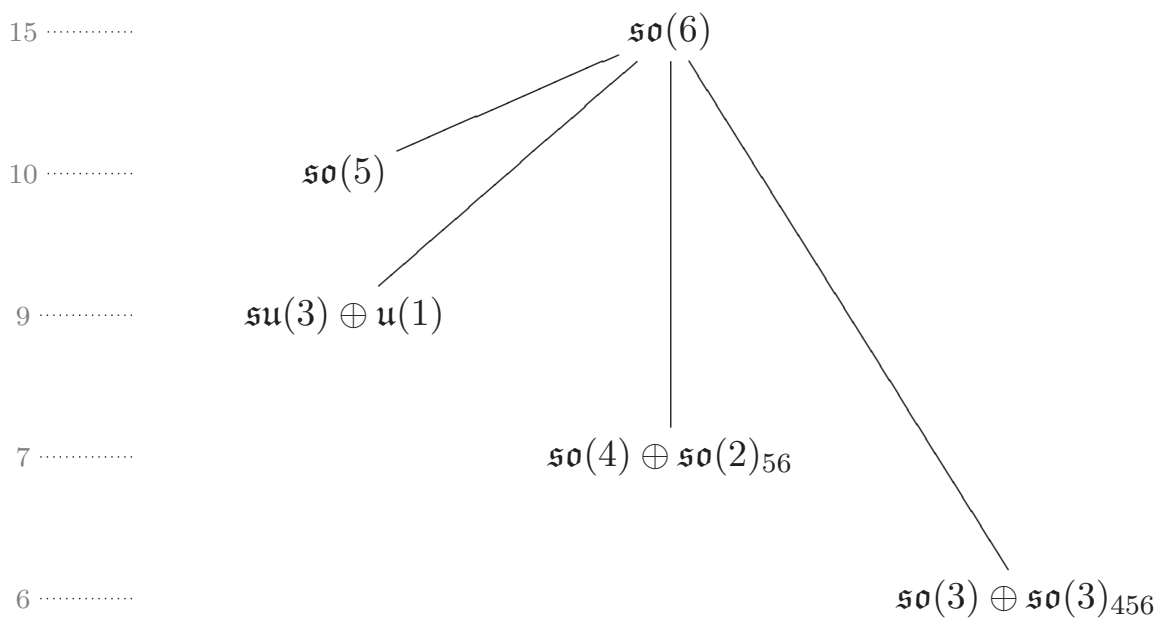


The subalgebra $\mathfrak{s u}(3) \oplus \mathfrak{u}(1)$ can be described explicitly as the centraliser of $L_{12}+L_{34}+$ $L_{56}$, which spans the $\mathfrak{u}(1)$ subalgebra of $\mathfrak{s u}(3) \oplus \mathfrak{u}(1)$. A basis for the $\mathfrak{s u}(3)$ subalgebra is given by

$$
\begin{array}{llll}
L_{13}+L_{24} & L_{14}-L_{23} & L_{15}+L_{26} & L_{16}-L_{25} \\
L_{35}+L_{46} & L_{36}-L_{45} & L_{12}-L_{34} & L_{34}-L_{56}
\end{array} .
$$

The Lie algebra $\mathfrak{s u}(3)$ has two inequivalent maximal subalgebras. First we have a regular subalgebra isomorphic to $\mathfrak{s u}(2) \oplus \mathfrak{u}(1)$. Up to equivalence, we may choose it to lie inside $\mathfrak{s o}(4)$ and corresponds to $\mathfrak{s o}(3)_{-} \oplus \mathfrak{s o}(2)_{+}$, which is itself equivalent to $\mathfrak{s o}(3)_{+} \oplus \mathfrak{s o}(2)_{-}$. The second inequivalent maximal subalgebra of $\mathfrak{s u}(3)$ is a singular subalgebra isomorphic to $\mathfrak{s o}(3)$ and denoted $\mathfrak{s o}(3)_{S}$. This subalgebra acts irreducibly on the fundamental 3dimensional representation and in fact consists of the real matrices in that representation. It follows that any of its proper subalgebras decomposes the fundamental representation of $\mathfrak{s u}(3)$ and this is why it is already contained in the other maximal subalgebra. The corresponding Hasse diagram is given by

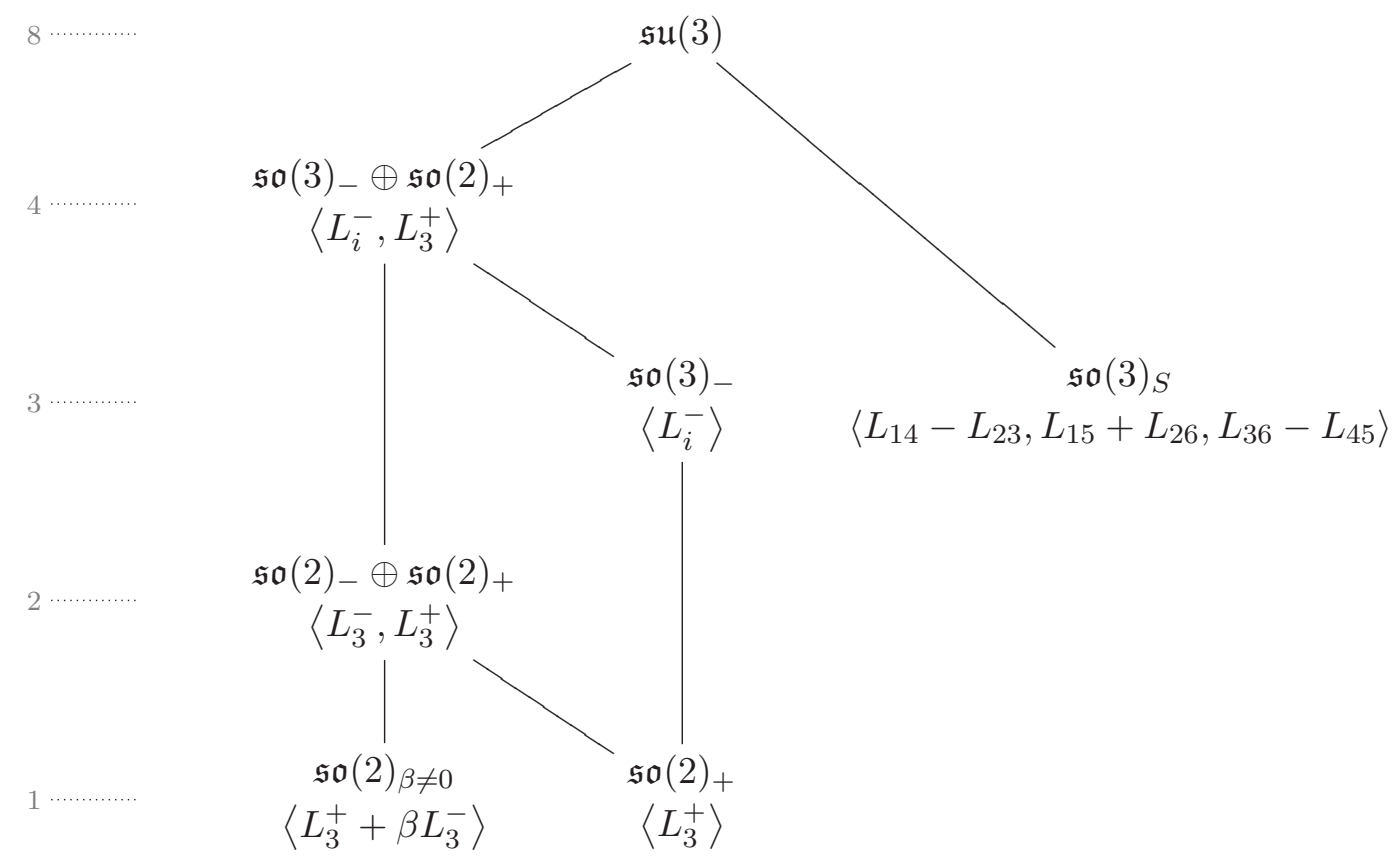

where we have omitted the $\mathfrak{s o}(2)$ subalgebra of $\mathfrak{s o}(3)_{S}$ since it does not coincide with any of the $\mathfrak{s o}(2)_{\beta}$ subalgebras, but only equivalent to $\mathfrak{s o}(2)_{-}$. In summary, the only subalgebra of $\mathfrak{s u}(3)$ which is not already (equivalent to) a subalgebra of $\mathfrak{s o}(4)$ is $\mathfrak{s o}(3)_{S}$.

However it is $\mathfrak{s u}(3) \oplus \mathfrak{u}(1)$ which is the maximal subalgebra of $\mathfrak{s o}(6)$ and it behoves us to classify its subalgebras. Goursat's Lemma guarantees that such subalgebras are fibered products $\mathfrak{h}_{L} \oplus_{\mathfrak{q}} \mathfrak{h}_{R}$, where $\mathfrak{h}_{L}<\mathfrak{s u}(3)$ and $\mathfrak{h}_{R}<\mathfrak{u}(1)$ and where $\operatorname{dim} \mathfrak{q}=0$ or 1 . In the former case, the subalgebras are direct products, whereas in the latter they are graphs of nonzero homomorphisms $\mathfrak{h}_{L} \rightarrow \mathfrak{s o}(2)$, where $\mathfrak{h}_{L}<\mathfrak{s u}(3)$ is one of the subalgebras 
admitting such homomorphisms. A compact Lie algebra admits a nonzero homomorphism to $\mathfrak{s o}(2)$ if and only if it has itself an $\mathfrak{s o}(2)$ factor. Of the subalgebras of $\mathfrak{s u}(3)$ with this property, all are contained in $\mathfrak{s o}(4)$ and hence they will be counted among the subalgebras of $\mathfrak{s o}(4) \oplus \mathfrak{s o}(2)_{56}$. The reason is that if $\mathfrak{h}_{L}<\mathfrak{s o}(4)$ then $\mathfrak{h}_{L} \oplus \mathfrak{u}(1)$ will be equivalent to a subalgebra of $\mathfrak{s o}(4) \oplus \mathfrak{s o}(2)_{56}$. Of the direct product subalgebras, all except for $\mathfrak{s o}(3)_{S}$ itself and $\mathfrak{s o}(3)_{S} \oplus \mathfrak{u}(1)$ are subalgebras of $\mathfrak{s o}(4) \oplus \mathfrak{s o}(2)_{56}$. An explicit basis for $\mathfrak{s o}(3)_{S} \oplus \mathfrak{u}(1)$ is given by

$$
\mathfrak{s o}(3)_{S} \oplus \mathfrak{u}(1)=\mathbb{R}\left\langle L_{14}-L_{23}, L_{15}+L_{26}, L_{36}-L_{45}, L_{12}+L_{34}+L_{56}\right\rangle
$$

It thus remains to determine the subalgebras of $\mathfrak{s o}(4) \oplus \mathfrak{s o}(2)_{56}$. Goursat's Lemma says that they are products of subalgebras of $\mathfrak{s o}(4)$ and $\mathfrak{s o}(2)_{56}$ fibered over some Lie algebra $\mathfrak{q}$. Since $\operatorname{dim} \mathfrak{s o}(2)_{56}=1, \operatorname{dim} \mathfrak{q} \leq 1$ and we have two cases to consider: $\operatorname{dim} \mathfrak{q}=0$, which corresponds to the case of direct products of subalgebras, and $\operatorname{dim} \mathfrak{q}=1$. In this latter case, the map $\mathfrak{s o}(2)_{56} \rightarrow \mathfrak{q}$ is an isomorphism, and thus the subalgebras are graphs of nonzero homomorphisms $\mathfrak{h}_{L} \rightarrow \mathfrak{s o}(2)_{56}$, where $\mathfrak{h}_{L}<\mathfrak{s o}(4)$ is a subalgebra admitting such homomorphisms. A quick glance at the Hasse diagram (4.3) for $\mathfrak{s o}(4)$ identifies such $\mathfrak{h}_{L}$ as one of $\mathfrak{s o}(3)_{+} \oplus \mathfrak{s o}(2)_{-}, \mathfrak{s o}(2)_{+} \oplus \mathfrak{s o}(2)_{-}$or $\mathfrak{s o}(2)_{\beta}$. The resulting subalgebras of $\mathfrak{s o}(4) \oplus \mathfrak{s o}(2)_{56}$ are explicitly given as follows:

- $\left(\mathfrak{s o}(3)_{+} \oplus \mathfrak{s o}(2)_{-}\right) \oplus_{\mathfrak{s o}(2)} \mathfrak{s o}(2)_{56}=\mathbb{R}\left\langle L_{i}^{+}, L_{3}^{-}+\alpha L_{56}\right\rangle, \alpha \neq 0$;

- $\left(\mathfrak{s o}(2)_{+} \oplus \mathfrak{s o}(2)_{-}\right) \oplus_{\mathfrak{s o}(2)} \mathfrak{s o}(2)_{56}=\mathbb{R}\left\langle L_{3}^{+}+\beta L_{56}, L_{3}^{-}+\alpha L_{56}\right\rangle,(\alpha, \beta) \in \mathbb{R}^{2}$ not both zero; and

- $\mathfrak{s o}(2)_{\beta} \oplus_{\mathfrak{s o}(2)} \mathfrak{s o}(2)_{56}=\left\langle L_{3}^{+}+\beta L_{3}^{-}+\alpha L_{56}\right\rangle, \beta \in(0,1)$ and $\alpha \neq 0$.

Among the product subalgebras, those which are contained in $\mathfrak{s o}(4)$ are already included inside $\mathfrak{s o}(5)$, so we must consider those of the form $\mathfrak{h} \oplus \mathfrak{s o}(2)_{56}$, with $\mathfrak{h}<\mathfrak{s o}(4)$, but only those which are not contained inside $\mathfrak{s o}(5)$; that is, those which do not leave any nonzero vector invariant in $\mathbb{R}^{6}$. A quick glance at the Hasse diagram (4.3) of subalgebras of $\mathfrak{s o}(4)$ reveals that the following product subalgebras of $\mathfrak{s o}(4) \oplus \mathfrak{s o}(2)_{56}$ have not appeared before:

- $\mathfrak{s o}(3)_{+} \oplus \mathfrak{s o}(2)_{-} \oplus \mathfrak{s o}(2)_{56}=\mathbb{R}\left\langle L_{i}^{+}, L_{3}^{-}, L_{56}\right\rangle$

- $\mathfrak{s o}(3)_{+} \oplus \mathfrak{s o}(2)_{56}=\mathbb{R}\left\langle L_{i}^{+}, L_{56}\right\rangle ;$

- $\mathfrak{s o}(2)_{+} \oplus \mathfrak{s o}(2)_{-} \oplus \mathfrak{s o}(2)_{56}=\mathbb{R}\left\langle L_{3}^{+}, L_{3}^{-}, L_{56}\right\rangle ;$ and

- $\mathfrak{s o}(2)_{0 \leq \beta<1} \oplus \mathfrak{s o}(2)_{56}=\mathbb{R}\left\langle L_{3}^{+}+\beta L_{3}^{-}, L_{56}\right\rangle$.

Finally, we consider the maximal subalgebra $\mathfrak{s o}(3) \oplus \mathfrak{s o}(3)_{456}$, which is isomorphic to $\mathfrak{s o}(4)$, but embedded in a different way in $\mathfrak{s o}(6)$. Being isomorphic to $\mathfrak{s o}(4)$, its subalgebras can be read (after some translation) from the Hasse diagram (4.3) for $\mathfrak{s o}(4)$. It is not hard to see that all subalgebras are already contained in at least one of the other maximal 
subalgebras of $\mathfrak{s o}(6)$. Indeed, the Hasse diagram of subalgebras for $\mathfrak{s o}(3) \oplus \mathfrak{s o}(3)_{456}$ is given by

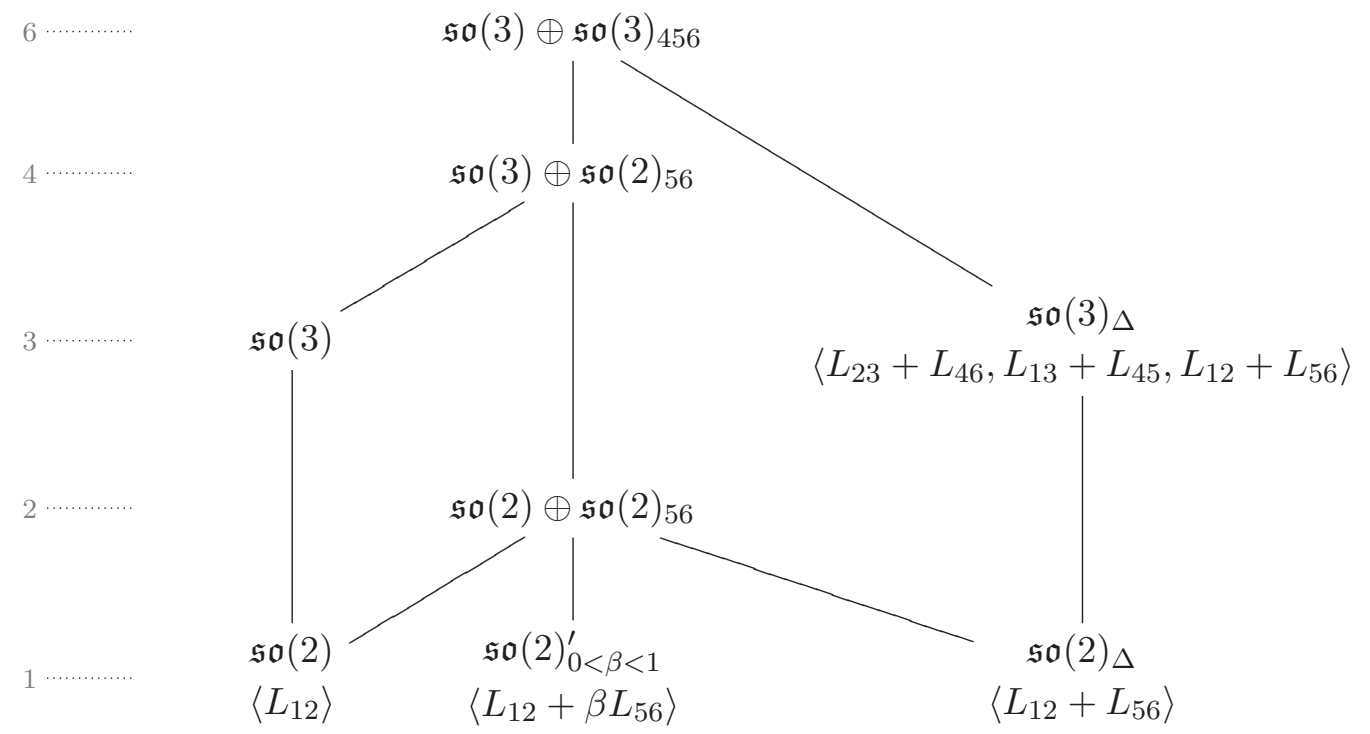

Hence we see that all but $\mathfrak{s o}(3)_{\Delta}$ are contained in $\mathfrak{s o}(4) \oplus \mathfrak{s o}(2)_{56}$, whereas it is not hard to see that $\mathfrak{s o}(3)_{\Delta}$ preserves a symplectic structure in $\mathbb{R}^{6}$ and hence it is contained in a $\mathfrak{u}(3)$ subalgebra of $\mathfrak{s o}(6)$. In fact, it is equivalent to the singular subalgebra $\mathfrak{s o}(3)_{S}$ of $\mathfrak{s u}(3)$.

In summary, a proper Lie subalgebra of $\mathfrak{s o}(6)$ is one of the following subalgebras, which have been described explicitly above:

- $\mathfrak{s o}(5)$ or one of its subalgebras, described in diagram (4.5),

- $\mathfrak{s o}(3) \oplus \mathfrak{s o}(3)_{456}=\mathbb{R}\left\langle L_{12}, L_{13}, L_{23}, L_{45}, L_{46}, L_{56}\right\rangle$,

- $\mathfrak{s u}(3) \oplus \mathfrak{u}(1)$ or one of the subalgebras:

$-\mathfrak{s o}(3)_{S} \oplus \mathfrak{u}(1)$, or

$-\mathfrak{s o}(3)_{S}$,

- $\mathfrak{s o}(4) \oplus \mathfrak{s o}(2)_{56}$ or one of the subalgebras:

$-\mathfrak{s o}(3)_{+} \oplus \mathfrak{s o}(2)_{-} \oplus \mathfrak{s o}(2)_{56}$,

$-\mathfrak{s o}(3)_{+} \oplus \mathfrak{s o}(2)_{56}$,

$-\mathfrak{s o}(2)_{+} \oplus \mathfrak{s o}(2)_{-} \oplus \mathfrak{s o}(2)_{56}$,

$-\mathfrak{s o}(2)_{0 \leq \beta<1} \oplus \mathfrak{s o}(2)_{56}$,

$-\left(\mathfrak{s o}(3)_{+} \oplus \mathfrak{s o}(2)_{-}\right) \oplus_{\mathfrak{s o}(2)} \mathfrak{s o}(2)_{56}$,

$-\left(\mathfrak{s o}(2)_{+} \oplus \mathfrak{s o}(2)_{-}\right) \oplus_{\mathfrak{s o}(2)} \mathfrak{s o}(2)_{56}$, or

$-\mathfrak{s o}(2)_{\beta} \oplus_{\mathfrak{s o}(2)} \mathfrak{s o}(2)_{56}$.

It is satisfying to find among these subalgebras precisely the four inequivalent $\mathfrak{s o}(3)$ subalgebras of $\mathfrak{s o}(6): \mathfrak{s o}(3)$ and $\mathfrak{s o}(3)_{+}$inside $\mathfrak{s o}(4), \mathfrak{s o}(3)_{\text {irr }}$ inside $\mathfrak{s o}(5)$ and $\mathfrak{s o}(3)_{S}$ inside $\mathfrak{u}(3)$. 


\subsection{Lie subalgebras of $\mathfrak{s o}(7)$}

The Lie algebra $\mathfrak{s o}(7)$ too has four inequivalent maximal subalgebras. Three of them decompose the 7-dimensional representation: namely, $\mathfrak{s o}(6), \mathfrak{s o}(5) \oplus \mathfrak{s o}(2)_{67}$ and $\mathfrak{s o}(4) \oplus \mathfrak{s o}(3)_{567}$; whereas one acts irreducibly: namely, $\mathfrak{g}_{2}$. The Lie algebra $\mathfrak{g}_{2}$ has three inequivalent maximal subalgebras: $\mathfrak{s u}(3)$ and $\mathfrak{s u}(2) \oplus \mathfrak{s u}(2)$, which decompose the 7-dimensional irreducible representation, and one acting irreducibly there: namely, $\mathfrak{s u}(2)_{\text {irr }}$. This yields the following subdiagram of the Hasse diagram of subalgebras of $\mathfrak{s o}(7)$.

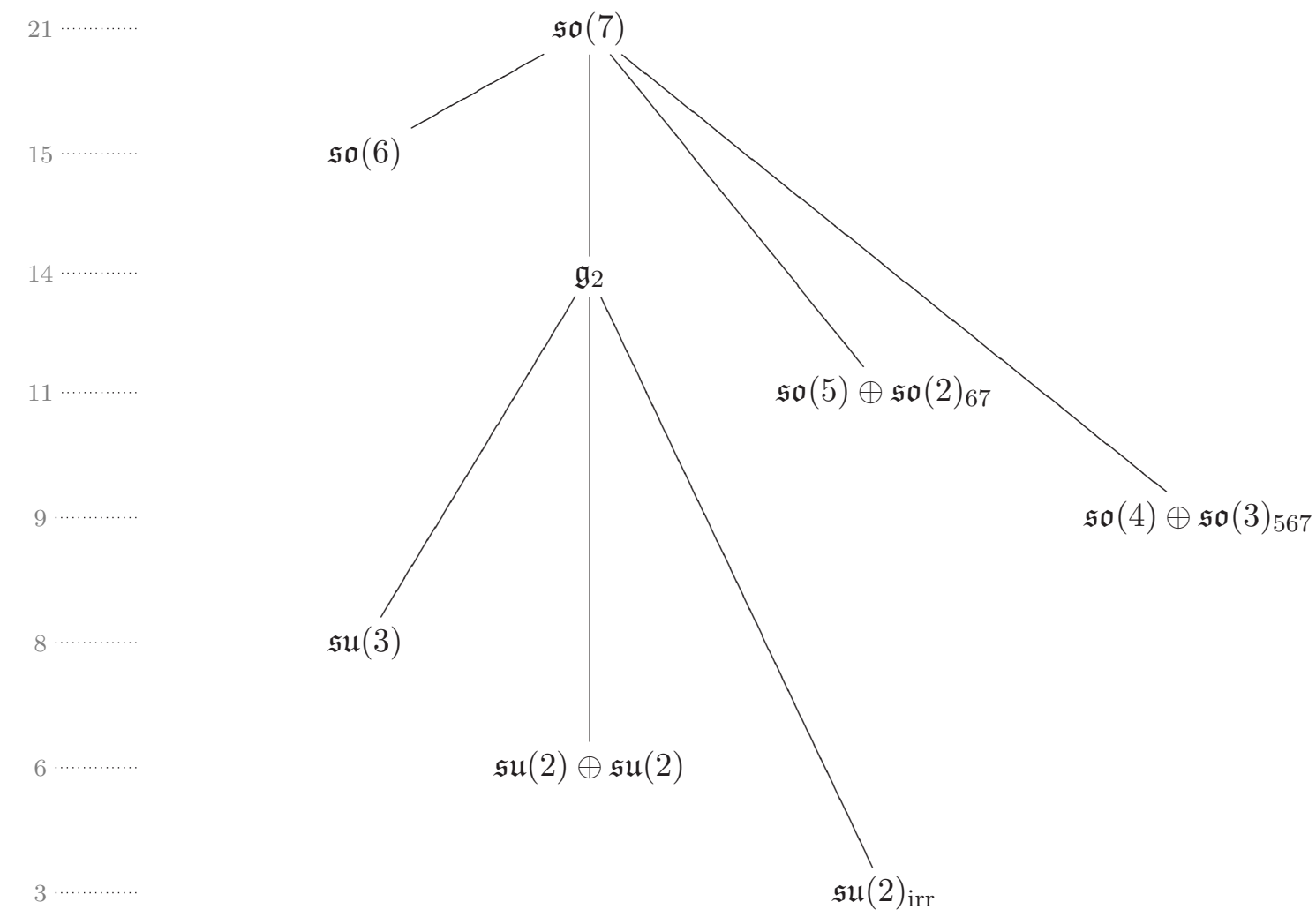

Although in order to fully specify the Hasse diagram for $\mathfrak{s o}(7)$ we would have to determine the subalgebras of $\mathfrak{s o}(5) \oplus \mathfrak{s o}(2)$ and $\mathfrak{s o}(4) \oplus \mathfrak{s o}(3)$, and as tempting as that is, it is also unnecessary for what follows. We record here an explicit basis for the $\mathfrak{g}_{2}$ subalgebra of $\mathfrak{s o}(7)$ :

$$
\begin{array}{lllllll}
L_{14}-L_{23} & L_{13}+L_{24} & L_{17}+L_{26} & L_{16}-L_{27} & L_{12}-L_{34} & L_{17}-L_{35} & L_{25}-L_{36} \\
L_{15}+L_{37} & L_{16}+L_{45} & L_{15}-L_{46} & L_{25}-L_{47} & L_{14}+L_{56} & L_{13}-L_{57} & L_{12}+L_{67}
\end{array}
$$

\section{The supergravity field equations for homogeneous backgrounds}

The above results allow us in principle to determine all eleven-dimensional lorentzian homogeneous spaces with a transitive action of a group $G$ locally isomorphic to $\mathrm{SO}(n) \times \mathrm{SO}(3,2)$. For each such lorentzian manifold, we wish to investigate whether there are any solutions to the supergravity field equations. The field equations are partial differential equations but they become algebraic in a homogeneous Ansatz, by which we mean that the 4-form is also $G$-invariant. In this section we will write down the field equations in a homogeneous Ansatz. 


\subsection{The field equations of eleven-dimensional supergravity}

Following the conventions of [3], the bosonic part of the action of $d=11$ supergravity is (setting Newton's constant to 1)

$$
\int_{M}\left(\frac{1}{2} R \mathrm{dvol}-\frac{1}{4} F \wedge \star F+\frac{1}{12} F \wedge F \wedge A\right),
$$

where $F=d A$ locally, $R$ is the scalar curvature of $g$ and dvol is the (signed) volume element

$$
\mathrm{dvol}:=\sqrt{|g|} d x^{0} \wedge d x^{1} \wedge \cdots \wedge d x^{10} .
$$

The Euler-Lagrange equations following from (5.1) are

$$
\begin{aligned}
d \star F & =\frac{1}{2} F \wedge F \\
\operatorname{Ric}(X, Y) & =\frac{1}{2}\left\langle\iota_{X} F, \iota_{Y} F\right\rangle-\frac{1}{6} g(X, Y)|F|^{2},
\end{aligned}
$$

for all vector fields $X, Y$ on $M$. In this equation we have introduced the inner product $\langle-,-\rangle$ on differential forms, defined by

$$
\langle\theta, \omega\rangle \mathrm{dvol}=\theta \wedge \star \omega,
$$

and the associated norm

$$
|\theta|^{2}=\langle\theta, \theta\rangle
$$

which in a lorentzian manifold is not positive-definite.

The field equations (5.3) are invariant under the homothetic action of $\mathbb{R}^{+}:(g, F) \mapsto$ $\left(e^{2 t} g, e^{3 t} F\right)$, where $t \in \mathbb{R}$. Indeed, under $g \mapsto e^{2 t} g$, the Levi-Civita connection, consisting of terms of the form $g^{-1} d g$, does not change. This means that the $(3,1)$ Riemann curvature tensor is similarly invariant, and so is any contraction such as the Ricci tensor. Under $F \mapsto e^{3 t} F$, the tensor in the right-hand side of the Einstein equation is similarly invariant, since the $e^{6 t}$ coming from the two $F$ s cancels the $e^{-6 t}$ coming from the three $g^{-1}$ s. On the other hand, the Bianchi identity $d F=0$ is clearly invariant under homotheties and the Maxwell-like equation is as well. Indeed, using that the Hodge $\star$ acting on $p$-forms in a $D$ dimensional manifold, scales like $e^{(D-2 p) t}$ under $g \mapsto e^{2 t} g$, we see that $\star$ acting on 4 -forms in 11-dimensions scales like $e^{3 t}$, just like $F$, whence both sides of the Maxwell-like equation scale in the same way: namely, $e^{6 t}$. This means that the moduli spaces of solutions of the field equations are always cones. It is possible to extend this to a homothetic action of $\mathbb{R}^{\times}$ (the nonzero real numbers) if we take the point of view that the vielbeins scale by $\lambda \neq 0$, whence if $\lambda<0$ the orientation changes. The particular homothety where $\lambda=-1$, which is just orientation reversal, is known as "skew-whiffing" in the early supergravity literature, as described for example in [18].

\subsection{The equivalent algebraic equations}

Let us assume that we are looking for homogeneous supergravity backgrounds. This means that the spacetime is a homogeneous eleven-dimensional lorentzian manifold $G / H$ and that the 4 -form $F$ is $G$-invariant. Algebraically, such a background is determined by a split 
$\mathfrak{g}=\mathfrak{h} \oplus \mathfrak{m}$ of the Lie algebra of $G$ into the Lie algebra of $H$ and a complement $\mathfrak{m}$. As explained in section 2.1, for $G$ semisimple we may restrict ourselves to the case where $\mathfrak{g}=\mathfrak{h} \oplus \mathfrak{m}$ is a reductive split.

Let us introduce bases $Y_{i}$ for $\mathfrak{m}$ and $X_{a}$ for $\mathfrak{h}$, relative to which the Lie brackets are given by

$$
\left[X_{a}, X_{b}\right]=f_{a b}{ }^{c} X_{c} \quad\left[X_{a}, Y_{i}\right]=f_{a i}{ }^{j} Y_{j} \quad \text { and } \quad\left[Y_{i}, Y_{j}\right]=f_{i j}{ }^{k} Y_{k}+f_{i j}{ }^{a} X_{a} .
$$

The metric is given by a lorentzian inner product, denoted $g$, on $\mathfrak{m}$ which is invariant under the linear isotropy representation of $\mathfrak{h}$ and with components $\left\langle Y_{i}, Y_{j}\right\rangle=g_{i j}$ relative to the chosen basis. The 4 -form is given by an element $F \in \Lambda^{4} \mathfrak{m}^{*}$ which is similarly invariant and has components $F\left(Y_{i}, Y_{j}, Y_{k}, Y_{l}\right)=F_{i j k l}$.

The data $(\mathfrak{g}, \mathfrak{h}, \mathfrak{m}, g, F)$ defines a homogeneous background of eleven-dimensional supergravity if and only if the following equations are satisfied:

- the Bianchi identity $d F=0$, which relative to the basis is given explicitly by setting expression (2.18) to zero;

- the nonlinear Maxwell equation

$$
\delta F=-\star \frac{1}{2} F \wedge F
$$

- and the Einstein equation

$$
R_{i j}=\frac{1}{12} F_{i k l m} F_{j}^{k l m}-\frac{1}{144} g_{i j} F_{k l m n} F^{k l m n},
$$

where $R_{i j}$ is given by equation $(2.14)$

\subsection{The methodology}

Let us now explain the method by which we search for homogeneous backgrounds. Having chosen $\mathfrak{g}=\mathfrak{h} \oplus \mathfrak{m}$ we first determine whether there is an $\mathfrak{h}$-invariant lorentzian inner product on $\mathfrak{m}$. As mentioned in section 2.1, for the case when $\mathfrak{h}$ is compact, this will be the case if and only if $\mathfrak{h}$ leaves invariant some nonzero vector in $\mathfrak{m}$; in other words, if $\mathfrak{m}^{\mathfrak{h}} \neq 0$, where $\mathfrak{m}^{\mathfrak{h}}$ denotes the subspace of $\mathfrak{m}$ which is fixed pointwise by $\mathfrak{h}$. If $\mathfrak{m}$ admits an $\mathfrak{h}$-invariant lorentzian inner product we say that $(\mathfrak{g}, \mathfrak{h}, \mathfrak{m})$ is admissible.

Let $(\mathfrak{g}, \mathfrak{h}, \mathfrak{m})$ be admissible. Then next step is to determine the (nontrivial) vector space $\left(S^{2} \mathfrak{m}^{*}\right)^{\mathfrak{h}}$ of $\mathfrak{h}$-invariant symmetric bilinear forms and the subset consisting of invariant lorentzian inner products. This subset will be an open subset of $\left(S^{2} \mathfrak{m}^{*}\right)^{\mathfrak{h}}$ and will thus be parametrised by $\operatorname{dim}\left(S^{2} \mathfrak{m}^{*}\right)^{\mathfrak{h}}$ parameters subject to some inequalities to ensure that the symmetric bilinear form is nondegenerate and has lorentzian signature. Clearly, it is a cone, since rescaling a lorentzian inner product by a positive real number yields another lorentzian inner product. Let $\left\{\gamma_{\alpha}\right\}$ denote the parameters associated to the inner product. Similarly we determine the vector space $\left(\Lambda^{4} \mathfrak{m}^{*}\right)^{\mathfrak{h}}$ of $\mathfrak{h}$-invariant 4 -forms on $\mathfrak{m}$ and the subspace consisting of closed 4-forms; namely, those obeying equation (2.18). Choosing a basis for the closed invariant 4 -forms, we can specify every such form by some parameters 
$\left\{\varphi_{\alpha}\right\}$. The Maxwell and Einstein equations then give a set of algebraic equations for the parameters $\gamma_{\alpha}$ and $\varphi_{\alpha}$ which we must solve. (They are in fact polynomial in $\varphi_{\alpha}$ and in $\sqrt{\gamma_{\alpha}}$.)

Two small simplifications can be made to reduce the number of free parameters. First of all, the homothety invariance of the equations allows us to eliminate one of the $\gamma_{\alpha}$ : if one of the $\gamma_{\alpha}$ is known to be different from zero, then we can assume that it has magnitude 1 via a homothety. Typically we will choose the $\gamma_{\alpha}$ corresponding to the timelike direction and set it equal to -1 .

The second simplification is a little more subtle and consists of exploiting the normaliser of $\mathfrak{h}$ in $\mathfrak{g}$ in order to eliminate one or more of the $\gamma_{\alpha}$ parameters. Let $\mathfrak{n}$ denote the normaliser of $\mathfrak{h}$ in $\mathfrak{g}$ : that is, the largest subalgebra of $\mathfrak{g}$ which contains $\mathfrak{h}$ as an ideal. More formally, we say that

$$
X \in \mathfrak{n} \Longleftrightarrow[X, Y] \in \mathfrak{h} \quad \forall Y \in \mathfrak{h} .
$$

Let $X \in \mathfrak{n}$. Since $\mathfrak{g}=\mathfrak{h} \oplus \mathfrak{m}$, we may decompose $X=X_{\mathfrak{h}}+X_{\mathfrak{m}}$ uniquely, where $X_{\mathfrak{h}} \in \mathfrak{h}$ and $X_{\mathfrak{m}} \in \mathfrak{m}$. Since $X \in \mathfrak{n}$, it obeys $[X, Y] \in \mathfrak{h}$ for all $Y \in \mathfrak{h}$, or equivalently $\left[X_{\mathfrak{h}}+X_{\mathfrak{m}}, Y\right] \in \mathfrak{h}$ for all $Y \in \mathfrak{h}$. Since $\mathfrak{h}$ is a subalgebra, $\left[X_{\mathfrak{h}}, Y\right] \in \mathfrak{h}$ and hence the only condition rests on $X_{\mathfrak{m}}:\left[X_{\mathfrak{m}}, Y\right] \in \mathfrak{h}$ for all $Y \in \mathfrak{h}$. However since the split is reductive, $\left[X_{\mathfrak{m}}, Y\right] \in \mathfrak{m}$ for all $Y \in \mathfrak{h}$ and hence it must happen that $\left[X_{\mathfrak{m}}, Y\right]=0$ for all $Y \in \mathfrak{h}$; in other words, $X_{\mathfrak{m}} \in \mathfrak{m}^{\mathfrak{h}}$. That is to say, the normaliser of $\mathfrak{h}$ in $\mathfrak{g}$ is given by $\mathfrak{n}=\mathfrak{h} \oplus \mathfrak{m}^{\mathfrak{h}}$. Let $N$ be the normaliser of $H$ in $G$, so that

$$
x \in N \Longleftrightarrow x y x^{-1} \in H \quad \forall y \in H .
$$

Then $N$ is a subgroup of $G$ with Lie algebra $\mathfrak{n}$. It is convenient to define the abstract group $W=N / H$, which is a group because $H$ is normal in $N$ by definition. The Lie algebra of $W$ is precisely $\mathfrak{m}^{\mathfrak{h}}$. Indeed, suppose that $X=X_{\mathfrak{h}}+X_{\mathfrak{m}} \in \mathfrak{g}$ belongs to the normaliser of $\mathfrak{h}$ in $\mathfrak{g}$. Then for all $Y \in \mathfrak{h},[X, Y] \in \mathfrak{h}$. This is equivalent to $\left[X_{\mathfrak{m}}, Y\right] \in \mathfrak{h}$ for all $Y \in \mathfrak{h}$, but since the split is reductive, $\left[X_{\mathfrak{m}}, Y\right]=0$ for all $Y \in \mathfrak{h}$, whence $X_{\mathfrak{m}} \in \mathfrak{m}^{\mathfrak{h}}$. In other words, the Lie algebra of the normaliser of $H$ in $G$ is $\mathfrak{h} \oplus \mathfrak{m}^{\mathfrak{h}}$, from where the claim follows.

We saw above that in the case where $H$ is compact, $\mathfrak{m}^{\mathfrak{l}}$ is nonzero if $G / H$ is to admit a homogeneous lorentzian metric, whence in that case $W$ is a Lie group of dimension at least one.

It turns out that $W$ may be used to reduce the number of parameters defining the lorentzian metrics in $G / H$.

The idea is the following. Let $o \in G / H$ be the origin; that is, any point with stability subgroup $H$; that is,

$$
x \in H \Longleftrightarrow x \cdot o=o .
$$

Let $x \in N$ and consider the point $o^{\prime}=x \cdot o$. We claim that $o^{\prime}$ also has stability subgroup $H$. Indeed,

$y \cdot o^{\prime}=o^{\prime} \Longleftrightarrow y x \cdot o=x \cdot o \Longleftrightarrow x^{-1} y x \cdot o=o \Longleftrightarrow x^{-1} y x \in H \Longleftrightarrow y \in x H x^{-1}=H$.

Now suppose that $\Theta$ is a $G$-invariant tensor field on $G / H$. As explained in section 2.1, $\Theta$ is determined uniquely by its value $\Theta_{o}$ at $o$ (or indeed at any point). Now $\Theta_{o}$ is a tensor in $\mathfrak{m}$ invariant under the linear isotropy representation of $\mathfrak{h}$. Now consider the value of $\Theta$ at the 
point $o^{\prime}$ defined above. Since $\Theta$ is a $G$-invariant tensor, $\Theta_{o^{\prime}}=\Theta_{x \cdot o}=x \cdot \Theta_{o}$, which is again an $\mathfrak{h}$-invariant tensor in $\mathfrak{m}$, since $o^{\prime}$ has stability subgroup $H$. In other words, the group $N$ acts on the space of $\mathfrak{h}$-invariant tensors in $\mathfrak{m}$. In fact, since the subgroup $H$ (assumed connected) of $N$ acts trivially, what we have is actually an action of $W=N / H$ on the $\mathfrak{h}$-invariant tensors. It is this action which we can use to bring the invariant tensor to a simpler form. The idea is that at another point $o^{\prime}$ with the same stabiliser, our tensor will take a simpler form and we could have been working at that point from the start.

We will now proceed to systematically explore the possible homogeneous backgrounds of $G=\mathrm{SO}(n) \times \mathrm{SO}(3,2)$, for $n>4$. We will only consider the case where $G$ acts effectively; although one could also study admissible $G / H=\mathrm{SO}(n) / K$, where $K$ is a closed subgroup of $\mathrm{SO}(n)$, so that $H=\mathrm{SO}(3,2) \times K$. By dimension these only exist for $n \geq 6$. One can rule out the existence of such backgrounds for $n=7$, but we have not completed the analysis of the $n=6$ backgrounds. This is of questionable interest, though, since (the dual of) a conformal field theory should have a nontrivial action of the conformal group.

\section{Homogeneous non-AdS backgrounds}

We now systematically explore the possible eleven-dimensional homogeneous spaces with infinitesimal data $(\mathfrak{g}, \mathfrak{h})$ with $\mathfrak{g}=\mathfrak{s o}(n) \oplus \mathfrak{s o}(3,2)$, with $n=4,5,6,7$, and $\mathfrak{h}<\mathfrak{g}$ the Lie algebra of a compact subgroup; that is $\mathfrak{h}<\mathfrak{s o}(n) \oplus \mathfrak{s o}(3) \oplus \mathfrak{s o}(2)$.

\subsection{Still no $n=7$ duals}

Here $\mathfrak{g}=\mathfrak{s o}(7) \oplus \mathfrak{s o}(3,2)$ has dimension 31 , whence we are looking for subalgebras $\mathfrak{h}$ of dimension 20. There are however none. Indeed, by Goursat's Lemma, every such subalgebra is given by Lie subalgebras $\mathfrak{h}_{L}<\mathfrak{s o}(7)$ and $\mathfrak{h}_{R}<\mathfrak{s o}(3) \oplus \mathfrak{s o}(2)$ fibered over a common quotient $\mathfrak{q}$. By the dimension formula (3.5), we have that

$$
\operatorname{dim} \mathfrak{h}=\operatorname{dim} \mathfrak{h}_{L}+\operatorname{dim} \mathfrak{h}_{R}-\operatorname{dim} \mathfrak{q} \leq \operatorname{dim} \mathfrak{h}_{L}+\operatorname{dim} \mathfrak{h}_{R} \leq \operatorname{dim} \mathfrak{h}_{L}+4,
$$

but also $\operatorname{dim} \mathfrak{h} \geq \operatorname{dim} \mathfrak{h}_{L}$ from formula (3.1). Since $\operatorname{dim} \mathfrak{s o}(7)=21$, we have to take a proper subalgebra $\mathfrak{h}_{L}<\mathfrak{s o}(7)$. It follows from the Hasse diagram (4.11) of maximal subalgebras of $\mathfrak{s o}(7)$, that $\operatorname{dim} \mathfrak{h}_{L} \leq 15$, whence from the first of the above inequalities $\operatorname{dim} \mathfrak{h} \leq 19$.

\subsection{No new $n=6$ duals}

Here $\mathfrak{g}=\mathfrak{s o}(6) \oplus \mathfrak{s o}(3,2)$ has dimension 25 , whence we are looking for subalgebras $\mathfrak{h}$ of dimension 14. By Goursat's Lemma, $\mathfrak{h}$ is given by subalgebras $\mathfrak{h}_{L}<\mathfrak{s o}(6)$ and $\mathfrak{h}_{R}<$ $\mathfrak{s o}(3) \oplus \mathfrak{s o}(2)$ fibered over a common quotient $\mathfrak{q}$. The dimension formula (3.5) says that

$$
\operatorname{dim} \mathfrak{h} \leq \operatorname{dim} \mathfrak{h}_{L}+\operatorname{dim} \mathfrak{h}_{R},
$$

but as before we cannot take $\mathfrak{h}_{L}=\mathfrak{s o}(6)$ since $\operatorname{dim} \mathfrak{s o}(6)=15>\operatorname{dim} \mathfrak{h}$, violating equation (3.1). So we have to take a proper subalgebra $\mathfrak{h}_{L}<\mathfrak{s o}(6)$. From the Hasse diagram (4.6) we see that the largest dimension of a proper subalgebra is 10, corresponding to $\mathfrak{s o}(5)$. By the above inequality, this is also the smallest dimension we could take, 
hence there is precisely one such subalgebra, with $\mathfrak{q}=0$ and hence a direct product: $\mathfrak{h}=\mathfrak{s o}(5) \oplus \mathfrak{s o}(3) \oplus \mathfrak{s o}(2)$. Being a product, the geometry is also a product, and we have a homogeneous space locally isometric to $\mathrm{SO}(6) / \mathrm{SO}(5) \times(\mathrm{SO}(3,2) / \mathrm{SO}(3) \times \mathrm{SO}(2))$. However this homogeneous space does not admit an invariant lorentzian metric. Indeed, in the first factor $\mathrm{SO}(6) / \mathrm{SO}(5)$ the linear isotropy representation is irreducible and in fact $\mathrm{SO}(6) / \mathrm{SO}(5)$ is locally isometric to the round $S^{5}$. As for the second factor, $\mathfrak{s o}(3,2)=\mathfrak{s o}(3) \oplus \mathfrak{s o}(2) \oplus \mathfrak{m}$, where $\mathfrak{m}=\mathbf{3} \otimes \mathbf{2}$ is the tensor product of the fundamental vectorial representations of $\mathfrak{s o}(3)$ and $\mathfrak{s o}(2)$. Since there is no invariant line, there is no $\mathfrak{s o}(3) \oplus \mathfrak{s o}(2)$-invariant lorentzian inner product on $\mathfrak{m}$.

\subsection{Possible new $n=5$ duals}

Here $\mathfrak{g}=\mathfrak{s o}(5) \oplus \mathfrak{s o}(3,2)$ has dimension 20, whence we are looking for subalgebras $\mathfrak{h}$ of dimension 9. From Goursat's Lemma, such a subalgebra will be given by two subalgebras $\mathfrak{h}_{L} \subset \mathfrak{s o}(5)$ and $\mathfrak{h}_{R} \subset \mathfrak{s o}(3) \oplus \mathfrak{s o}(2)$ (the maximal compact subalgebra of $\mathfrak{s o}(3,2)$ ) fibered over a common quotient $\mathfrak{q}$. Again we have to take a proper subalgebra $\mathfrak{h}_{L}<\mathfrak{s o}(5)$, since $\operatorname{dim} \mathfrak{s o}(5)>9$. The Hasse diagram (4.5) of subalgebras of $\mathfrak{s o}(5)$ identifies precisely one such possible $\mathfrak{h}_{L}$ which obeys the inequality $9 \leq \operatorname{dim} \mathfrak{h}_{L}+\operatorname{dim} \mathfrak{h}_{R} \leq \operatorname{dim} \mathfrak{h}_{L}+4$ : namely, $\mathfrak{h}_{L}=\mathfrak{s o}(4)$. This means that $\mathfrak{q}=0$ since although $\mathfrak{s o}(4)$ is not simple, the smallest nonzero quotient has dimension 3 and that results in $\mathfrak{h}$ not of enough dimension. This in turn forces $\operatorname{dim} \mathfrak{h}_{R}=3$, whence $\mathfrak{h}_{R}$ is isomorphic to an $\mathfrak{s o}(3)$ subalgebra of $\mathfrak{s o}(3,2)$. Therefore, up to equivalence, there is precisely one choice for $\mathfrak{h}$ : namely, $\mathfrak{s o}(4) \oplus \mathfrak{s o}(3)$. The geometry will also therefore be locally isometric to a product: $\mathrm{SO}(5) / \mathrm{SO}(4) \times \mathrm{SO}(3,2) / \mathrm{SO}(3)$. The first factor is locally isometric to the round $S^{4}$ and the second factor now does possess an invariant lorentzian metric. Indeed, $\mathfrak{s o}(3,2)=\mathfrak{s o}(3) \oplus \mathfrak{p}$, where $\mathfrak{p} \cong \mathbb{R}^{3} \oplus \mathbb{R}^{3} \oplus \mathbb{R}$ decomposes under the linear isotropy representation as two copies of the three-dimensional vector representation of $\mathfrak{s o}(3)$ and a one-dimensional trivial representation.

Let $L_{a b}, L_{a 5}$ denote the standard generators of $\mathfrak{s o}(5)$, where $a, b=1,2,3,4$ and let $J_{i j}, J_{i A}, J_{45}$ denote the standard generators of $\mathfrak{s o}(3,2)$, where $i, j=1,2,3$ and $A=4,5$. Then $\mathfrak{h}$ is spanned by $L_{a b}, J_{i j}$ and $\mathfrak{m}$ by $L_{a 5}, J_{i A}, J_{45}$. The $L_{a 5}$ transform as a vector of $\mathfrak{s o}(4)=\mathbb{R}\left\langle L_{a b}\right\rangle$, whereas $J_{i A}$ transform as two copies of the vector representation of $\mathfrak{s o}(3)=\mathbb{R}\left\langle J_{i j}\right\rangle$. The index $A$ is a vector of the $\mathfrak{s o}(2)$ with generator $J_{45}$ which is the nontrivial part of the normaliser of $\mathfrak{h}$ in $\mathfrak{g}$. There is a 5 -parameter family of invariant lorentzian inner products on $\mathfrak{m}$ :

$$
\left\langle J_{45}, J_{45}\right\rangle=\gamma_{0}, \quad\left\langle L_{a 5}, L_{b 5}\right\rangle=\gamma_{1} \delta_{a b}, \quad\left\langle J_{i A}, J_{j B}\right\rangle=\delta_{i j} \Omega_{A B}
$$

where $\gamma_{0}<0, \gamma_{1}>0$ and $\Omega_{A B}$ is a positive-definite symmetric $2 \times 2$ matrix. The $\mathrm{SO}(2)$ subgroup generated by $J_{45}$ acts by rotating the basis $J_{i A}$. Let $R_{\vartheta} \in \mathrm{SO}(2)$ denote the

rotation by an angle $\vartheta$. Then the matrix $\Omega$ transforms as $\Omega \mapsto R_{\vartheta}^{T} \Omega R_{\vartheta}$. The off-diagonal component $\Omega_{12}$ transforms as

$$
\Omega_{12} \mapsto \frac{1}{2}\left(\Omega_{11}-\Omega_{22}\right) \sin 2 \vartheta+\Omega_{12} \cos 2 \vartheta
$$


If $\Omega_{12} \neq 0$, simply let $\vartheta \in(0, \pi / 2)$ be given by

$$
\vartheta=\frac{1}{2} \cot ^{-1}\left(\frac{\Omega_{22}-\Omega_{11}}{2 \Omega_{12}}\right) .
$$

With this choice, the transformed $\Omega$ is diagonal. Therefore, without loss of generality, we can assume that $\left\langle J_{i 4}, J_{j 5}\right\rangle=0$ and that

$$
\left\langle J_{i 4}, J_{j 4}\right\rangle=\gamma_{2} \delta_{i j} \quad \text { and } \quad\left\langle J_{i 5}, J_{j 5}\right\rangle=\gamma_{3} \delta_{i j}
$$

where $\gamma_{2}$ and $\gamma_{3}$ are positive. Furthermore, using the homothety invariance of the equations of motion, we can set $\gamma_{0}=-1$ without loss of generality. This then leaves three positive parameters $\gamma_{1,2,3}$ for the metric.

In order to compute the curvature, we need to compute the $U$ tensor. Since $S^{4}$ is a symmetric space, the $U$ tensor has no legs along the subspace spanned by the $L_{a 5}$. It is then not too hard to show, using equation (2.9), that all other components vanish except for the following:

$$
U\left(J_{i 4}, J_{j 5}\right)=\frac{1}{2}\left(\gamma_{3}-\gamma_{2}\right) \delta_{i j} J_{45}, \quad U\left(J_{45}, J_{i 4}\right)=\frac{1-\gamma_{2}}{2 \gamma_{3}} J_{i 5} \text { and } \quad U\left(J_{45}, J_{i 5}\right)=\frac{\gamma_{3}-1}{2 \gamma_{2}} J_{i 4} .
$$

Defining $\Lambda=1-\left(\gamma_{2}+\gamma_{3}\right)^{2}$, one computes the following nonzero components of the Ricci tensor:

$$
\begin{array}{ll}
\operatorname{Ric}\left(J_{45}, J_{45}\right)=6+\frac{3 \Lambda}{2 \gamma_{2} \gamma_{3}} & \operatorname{Ric}\left(J_{i 4}, J_{j 4}\right)=\left(\left(\gamma_{2}+\gamma_{3}\right)+\frac{\Lambda}{2 \gamma_{3}}-3\right) \delta_{i j} \\
\operatorname{Ric}\left(L_{a 5}, L_{b 5}\right)=3 \delta_{a b} & \operatorname{Ric}\left(J_{i 5}, J_{j 5}\right)=\left(\left(\gamma_{2}+\gamma_{3}\right)+\frac{\Lambda}{2 \gamma_{2}}-3\right) \delta_{i j},
\end{array}
$$

whence the Ricci scalar becomes

$$
R=\left(6+\frac{3 \Lambda}{2 \gamma_{2} \gamma_{3}}\right)\left(\gamma_{2}+\gamma_{3}-1\right)-6
$$

The space of invariant 4-forms is six-dimensional. A possible basis is given by the following 4 -forms. Firstly, we have the volume form on the $S^{4}$, which is given algebraically by

$$
L_{15}^{*} \wedge L_{25}^{*} \wedge L_{35}^{*} \wedge L_{45}^{*}
$$

We then have an invariant 2-form

$$
\omega=\sum_{i=1}^{3} J_{i 4}^{*} \wedge J_{i 5}^{*}
$$

and squaring it we get an invariant 4-form. Finally we have

$$
\sum_{i, j, k=1}^{3} \sum_{A, B, C=4}^{5} t_{A B C} \varepsilon_{i j k} J_{i A}^{*} \wedge J_{j B}^{*} \wedge J_{k C}^{*} \wedge J_{45}^{*},
$$


where $t_{A B C}$ is a symmetric 3 -tensor, whence it has four components. It turns out that all invariant 4-forms are already closed, so the Bianchi identity is identically satisfied in this homogeneous Ansatz. (This is not always the case, though.) An explicit basis for the space of invariant closed 4 -forms is then given by the following six 4 -forms:

$$
\begin{aligned}
& \Phi_{1}=L_{15}^{*} \wedge L_{25}^{*} \wedge L_{35}^{*} \wedge L_{45}^{*} \\
& \Phi_{2}=J_{45}^{*} \wedge J_{14}^{*} \wedge J_{24}^{*} \wedge J_{34}^{*} \\
& \Phi_{3}=J_{45}^{*} \wedge J_{15}^{*} \wedge J_{25}^{*} \wedge J_{35}^{*} \\
& \Phi_{4}=J_{45}^{*} \wedge\left(J_{14}^{*} \wedge J_{24}^{*} \wedge J_{35}^{*}+J_{14}^{*} \wedge J_{25}^{*} \wedge J_{34}^{*}+J_{15}^{*} \wedge J_{24}^{*} \wedge J_{34}^{*}\right) \\
& \Phi_{5}=J_{45}^{*} \wedge\left(J_{14}^{*} \wedge J_{25}^{*} \wedge J_{35}^{*}+J_{15}^{*} \wedge J_{24}^{*} \wedge J_{35}^{*}+J_{15}^{*} \wedge J_{25}^{*} \wedge J_{34}^{*}\right) \\
& \Phi_{6}=-J_{14}^{*} \wedge J_{15}^{*} \wedge J_{24}^{*} \wedge J_{25}^{*}-J_{14}^{*} \wedge J_{15}^{*} \wedge J_{34}^{*} \wedge J_{35}^{*}-J_{24}^{*} \wedge J_{25}^{*} \wedge J_{34}^{*} \wedge J_{35}^{*}
\end{aligned}
$$

whence the most general invariant closed 4 -form is $F=\sum_{\alpha=1}^{6} \varphi_{\alpha} \Phi_{\alpha}$. The Maxwell and Einstein equations now become algebraic equations on the 9 real parameters $\gamma_{1,2,3}>0$ and $\varphi_{1, \ldots, 6}$.

It is convenient to analyse these equations to choose an ordered basis $\left(X_{\mu}\right)_{\mu=0,1, \ldots, 9, \natural}$ for $\mathfrak{m}$ :

$$
X_{\mu}=\left(J_{45}, L_{15}, \ldots, L_{45}, J_{14}, \ldots, J_{34}, J_{15}, \ldots, J_{35}\right),
$$

with corresponding canonical dual basis $\theta^{\mu}$ for $\mathfrak{m}^{*}$. Then the inner product is given by

$$
g=-\left(\theta^{0}\right)^{2}+\gamma_{1}\left(\left(\theta^{1}\right)^{2}+\cdots+\left(\theta^{4}\right)^{2}\right)+\gamma_{2}\left(\left(\theta^{5}\right)^{2}+\cdots+\left(\theta^{7}\right)^{2}\right)+\gamma_{3}\left(\left(\theta^{8}\right)^{2}+\cdots+\left(\theta^{\natural}\right)^{2}\right),
$$

and the most general closed 4-form by

$$
\begin{aligned}
F= & \varphi_{1} \theta^{1234}+\varphi_{2} \theta^{0567}+\varphi_{3} \theta^{089 \natural}+\varphi_{4}\left(\theta^{056 \natural}-\theta^{0579}+\theta^{0678}\right) \\
& +\varphi_{5}\left(\theta^{059 \natural}-\theta^{068 \natural}+\theta^{0789}\right)+\varphi_{6}\left(\theta^{5689}+\theta^{578 \natural}+\theta^{679 \natural}\right) .
\end{aligned}
$$

It follows that if we let $F=\varphi_{1} \theta^{1234}+\bar{F}$, then $\frac{1}{2} F \wedge F=\varphi_{1} \theta^{1234} \wedge \bar{F}$. In addition, from equation $(2.20)$, one finds that

$$
\begin{aligned}
\delta F= & -\frac{3 \gamma_{2} \varphi_{4}}{\gamma_{3}} \theta^{567}+\frac{3 \gamma_{3} \varphi_{5}}{\gamma_{2}} \theta^{89 \natural}+\frac{2 \varphi_{6}}{\gamma_{2} \gamma_{3}}\left(\theta^{058}+\theta^{069}+\theta^{07 \natural}\right) \\
& +\left(\frac{\gamma_{3} \varphi_{2}}{\gamma_{2}}-\frac{2 \gamma_{2} \varphi_{5}}{\gamma_{3}}\right)\left(\theta^{56 \natural}-\theta^{579}+\theta^{678}\right)+\left(\frac{2 \gamma_{3} \varphi_{4}}{\gamma_{2}}-\frac{\gamma_{2} \varphi_{3}}{\gamma_{3}}\right)\left(\theta^{59 \natural}-\theta^{68 \natural}+\theta^{789}\right) .
\end{aligned}
$$

We note en passant that, as expected, the only invariant harmonic 4-form is proportional to the volume form on $S^{4}$ : namely, $\varphi_{1} \theta^{1234}$.

The nonlinear Maxwell equation is equation (5.7). In order to compute the Hodge $\star$ it is perhaps better to work with an orthonormal coframe $\bar{\theta}^{\mu}$, where

$$
\bar{\theta}^{\mu}= \begin{cases}\theta^{0} & \mu=0 \\ \frac{1}{\sqrt{\gamma}} \theta^{\mu} & \mu \in\{1,2,3,4\}, \\ \frac{1}{\sqrt{\gamma_{2}}} \theta^{\mu} & \mu \in\{5,6,7\}, \\ \frac{1}{\sqrt{\gamma_{3}}} \theta^{\mu} & \mu \in\{8,9, \text { দ }\},\end{cases}
$$


where we choose the positive square roots of the positive quantities $\gamma_{i}$. A short calculation later, one finds that

$$
\begin{aligned}
-\star \frac{1}{2} F \wedge F= & -\frac{\gamma_{2}^{3 / 2} \varphi_{1} \varphi_{3}}{\gamma_{1}^{2} \gamma_{3}^{3 / 2}} \theta^{567}+\frac{\gamma_{3}^{3 / 2} \varphi_{1} \varphi_{2}}{\gamma_{1}^{2} \gamma_{2}^{3 / 2}} \theta^{89 \natural}-\frac{\varphi_{1} \varphi_{6}}{\gamma_{1}^{2} \gamma_{2}^{1 / 2} \gamma_{3}^{1 / 2}}\left(\theta^{058}+\theta^{069}+\theta^{07 \natural}\right) \\
& +\frac{\gamma_{2}^{1 / 2} \varphi_{1} \varphi_{5}}{\gamma_{1}^{2} \gamma_{3}^{1 / 2}}\left(\theta^{56 \natural}-\theta^{579}+\theta^{678}\right)-\frac{\gamma_{3}^{1 / 2} \varphi_{1} \varphi_{4}}{\gamma_{1}^{2} \gamma_{2}^{1 / 2}}\left(\theta^{59 \natural}-\theta^{68 \natural}+\theta^{789}\right),
\end{aligned}
$$

which can be readily compared with equation (6.17) in order to arrive at the algebraic Maxwell equations:

$$
\begin{array}{rlrl}
\varphi_{4} & =\frac{\gamma_{2}^{1 / 2} \varphi_{1} \varphi_{3}}{3 \gamma_{1}^{2} \gamma_{3}^{1 / 2}} & \frac{\gamma_{2} \varphi_{3}}{\gamma_{3}} & =\left(\frac{2 \gamma_{3}}{\gamma_{2}}+\frac{\gamma_{3}^{1 / 2} \varphi_{1}}{\gamma_{1}^{2} \gamma_{2}^{1 / 2}}\right) \varphi_{4} \\
\varphi_{5} & =\frac{\gamma_{3}^{1 / 2} \varphi_{1} \varphi_{2}}{3 \gamma_{1}^{2} \gamma_{2}^{1 / 2}} & \frac{\gamma_{3} \varphi_{2}}{\gamma_{2}} & =\left(\frac{2 \gamma_{2}}{\gamma_{3}}+\frac{\gamma_{2}^{1 / 2} \varphi_{1}}{\gamma_{1}^{2} \gamma_{3}^{1 / 2}}\right) \varphi_{5} \\
0 & =\left(\frac{\varphi_{1}}{\gamma_{1}^{2}}+\frac{2}{\gamma_{2}^{1 / 2} \gamma_{3}^{1 / 2}}\right) \varphi_{6} . &
\end{array}
$$

The bottom equation defines two main branches of solutions, depending on whether $\varphi_{6}$ vanishes. The first two equations express $\varphi_{4}$ and $\varphi_{5}$ in terms of $\varphi_{3}$ and $\varphi_{2}$, respectively; whereas the remaining equations become:

$$
\varphi_{3}=\frac{1}{3}\left(\frac{2 \gamma_{3}}{\gamma_{2}}+\frac{\gamma_{3}^{1 / 2} \varphi_{1}}{\gamma_{1}^{2} \gamma_{2}^{1 / 2}}\right) \frac{\gamma_{3}^{1 / 2} \varphi_{1}}{\gamma_{1}^{2} \gamma_{2}^{1 / 2}} \varphi_{3} \quad \text { and } \quad \varphi_{2}=\frac{1}{3}\left(\frac{2 \gamma_{2}}{\gamma_{3}}+\frac{\gamma_{2}^{1 / 2} \varphi_{1}}{\gamma_{1}^{2} \gamma_{3}^{1 / 2}}\right) \frac{\gamma_{2}^{1 / 2} \varphi_{1}}{\gamma_{1}^{2} \gamma_{3}^{1 / 2}} \varphi_{2}
$$

Notice the invariance of the equations under the simultaneous exchanges: $\gamma_{2} \leftrightarrow \gamma_{3}$ and $\varphi_{2} \leftrightarrow \varphi_{3}$. This is nothing but the remnant of the action of the normaliser of $\mathfrak{h}$ in $\mathfrak{g}$, which our choice of diagonal inner product broke down to a $\mathbb{Z} / 2 \mathbb{Z}$ exchanging the 4 and 5 labels in $\mathfrak{s o}(3,2)$. This discrete symmetry relates some of the branches below.

Each of these equations also defines two branches, depending on whether $\varphi_{3}$ and $\varphi_{2}$ vanish or not. In all, we have 8 branches of solutions, two pairs of which are related by the remaining $\mathbb{Z} / 2 \mathbb{Z}$ action mentioned above. They are given as follows:

1. $\varphi_{2}=\varphi_{3}=\varphi_{6}=0$. This implies that $\varphi_{i \neq 1}=0$ and $\varphi_{1}$ remains free.

2. $\varphi_{2}=\varphi_{6}=0$, but $\varphi_{3}$ remains free. Then there are two sub-branches, distinguished by the choice of sign in

$$
\frac{\varphi_{1}}{\gamma_{1}^{2}}=-\sqrt{\frac{\gamma_{3}}{\gamma_{2}}} \pm \sqrt{\frac{\gamma_{3}}{\gamma_{2}}+3 \frac{\gamma_{2}}{\gamma_{3}}}
$$

3. $\varphi_{3}=\varphi_{6}=0$, but $\varphi_{2}$ remains free, and again two sub-branches distinguished by the sign in

$$
\frac{\varphi_{1}}{\gamma_{1}^{2}}=-\sqrt{\frac{\gamma_{2}}{\gamma_{3}}} \pm \sqrt{\frac{\gamma_{2}}{\gamma_{3}}+3 \frac{\gamma_{3}}{\gamma_{2}}}
$$


4. $\varphi_{6}=0$, but $\varphi_{2}, \varphi_{3}$ remain free. In this case, symmetry says that $\gamma_{2}=\gamma_{3}$, and hence we have two sub-branches:

(a) $\varphi_{1}=\gamma_{1}^{2}, \varphi_{4}=\varphi_{3} / 3, \varphi_{5}=\varphi_{2} / 3$, and

(b) $\varphi_{1}=-3 \gamma_{1}^{2}, \varphi_{4}=-\varphi_{3}, \varphi_{5}=-\varphi_{2}$.

5. $\varphi_{2}=\varphi_{3}=0$, but $\varphi_{6}$ remains free. This implies that $\varphi_{1}=-2 \gamma_{1}^{2} / \sqrt{\gamma_{2} \gamma_{3}}$.

6. $\varphi_{2}=0$, but $\varphi_{3}, \varphi_{6}$ remain free. Then $\varphi_{1}=-2 \gamma_{1}^{2} / \sqrt{\gamma_{2} \gamma_{3}}$ and then $\gamma_{3}=1-\frac{3}{4} \gamma_{2}^{2}$.

7. $\varphi_{3}=0$, but $\varphi_{2}, \varphi_{6}$ remain free. Again $\varphi_{1}=-2 \gamma_{1}^{2} / \sqrt{\gamma_{2} \gamma_{3}}$ and then $\gamma_{2}=1-\frac{3}{4} \gamma_{2}^{3}$.

8. $\varphi_{2}, \varphi_{3}, \varphi_{6}$ remain free. In this case, symmetry dictates (and one can also check) that $\gamma_{2}=\gamma_{3}=\frac{2}{3}$, whence $\varphi_{1}=-3 \gamma_{1}^{2}$.

The Einstein equations become five algebraic equations on the $\gamma_{i}$ and the $\varphi_{i}$. We may use two of the Maxwell equations in (6.20) to eliminate $\varphi_{4}$ and $\varphi_{5}$ from the equations and we may use that $\gamma_{1} \gamma_{2} \gamma_{3} \neq 0$ to clear denominators and arrive after some simplification at the following (almost) polynomial equations:

$$
\begin{aligned}
0= & \varphi_{1} \varphi_{2} \varphi_{3}\left(3 \gamma_{1}^{2}\left(\gamma_{2}+\gamma_{3}\right)+2 \sqrt{\gamma_{2} \gamma_{3}} \varphi_{1}\right) \\
0= & 3 \gamma_{1}^{4} \varphi_{6}^{2}+\gamma_{2}^{2} \gamma_{3}^{2}\left(12 \gamma_{1}^{3}-\varphi_{1}^{2}\right)+3 \gamma_{1}^{4} \gamma_{2} \gamma_{3}\left(\left(\gamma_{2}-\gamma_{3}\right)^{2}-1\right) \\
0= & \left(3 \gamma_{2} \gamma_{1}^{4}+\gamma_{3} \varphi_{1}^{2}\right) \gamma_{2}^{2} \varphi_{3}^{2}+\left(3 \gamma_{3} \gamma_{1}^{4}+\gamma_{2} \varphi_{1}^{2}\right) \gamma_{3}^{2} \varphi_{2}^{2}-9 \gamma_{1}^{4} \gamma_{2} \gamma_{3} \varphi_{6}^{2}+6 \gamma_{2}^{3} \gamma_{3}^{3} \varphi_{1}^{2}-54 \gamma_{1}^{3} \gamma_{2}^{3} \gamma_{3}^{3} \\
0= & 6 \gamma_{1}^{4} \gamma_{2}^{3} \varphi_{3}^{2}+\left(\gamma_{2} \varphi_{1}^{2}-3 \gamma_{1}^{4} \gamma_{3}\right) \gamma_{3}^{2} \varphi_{2}^{2}-9 \gamma_{1}^{4} \gamma_{2} \gamma_{3} \varphi_{6}^{2}+3 \gamma_{2}^{3} \gamma_{3}^{3} \varphi_{1}^{2}+9 \gamma_{1}^{4} \gamma_{2}^{2} \gamma_{3}^{2}\left(\gamma_{2}^{2}-\gamma_{3}^{2}-6 \gamma_{2}+1\right) \\
0= & 6 \gamma_{1}^{4} \gamma_{3}^{3} \varphi_{2}^{2}+\left(\gamma_{3} \varphi_{1}^{2}-3 \gamma_{1}^{4} \gamma_{2}\right) \gamma_{2}^{2} \varphi_{3}^{2}-9 \gamma_{1}^{4} \gamma_{2} \gamma_{3} \varphi_{6}^{2}+3 \gamma_{2}^{3} \gamma_{3}^{3} \varphi_{1}^{2} \\
& +9 \gamma_{1}^{4} \gamma_{2}^{2} \gamma_{3}^{2}\left(\gamma_{3}^{2}-\gamma_{2}^{2}-6 \gamma_{3}+1\right) .
\end{aligned}
$$

Notice that the first three equations are invariant under the remnant $\mathbb{Z} / 2 \mathbb{Z}$ symmetry, whereas the last two equations are mapped into each other.

We now insert each of the solution branches of the Maxwell equations in turn into the Einstein equations. We have used a mixture of symbolic and numerical computation to arrive at the following results, where the enumeration coincides with that of the solutions of the Maxwell equations.

1. We find one solution: $\gamma_{2}=\gamma_{3}=\frac{2}{3}, \gamma_{1}=\frac{4}{9}$ and $\varphi_{1}= \pm \frac{8}{9}$. This is a Freund-Rubin background, since the 4 -form is proportional to the volume form on the $S^{4}$. We will see below that the 7-dimensional geometry is that of a lorentzian Sasaki-Einstein manifold, whence this background is supersymmetric. This geometry will be studied in detail in section 8.2.1, where we will show that it is Wick-related to a known Freund-Rubin $\mathrm{AdS}_{4}$ background.

2. There are two branches, distinguished by the sign of the root in $\varphi_{1}$.

(+) In the positive branch, we find the following numerical solution:

$$
\begin{aligned}
& \gamma_{1}=0.22776420155467458 \\
& \gamma_{2}=0.4670546272324634 \\
& \gamma_{3}=0.12728016028858763 \\
& \text { whence } \\
& \varphi_{1}=0.14715771499261474 \\
& \varphi_{3}= \pm 0.27380714065085027 \text {. }
\end{aligned}
$$


We do not discard the possibility that one can do better and write this solution in some iterated quadratic extension of the rationals, but we have not been able to do it. The source of the difficulty comes from the fact that the solutions are built out of roots of a sixth order integer polynomial and we do not know if its Galois group is solvable.

(-) In the negative branch, we find the following solution

$$
\gamma_{1}=\gamma_{2}=\gamma_{3}=\frac{1}{3} \quad \varphi_{1}=-\frac{1}{3} \quad \text { and } \quad \varphi_{3}= \pm \frac{1}{\sqrt{3}} .
$$

We will see that this is part of a more general solution.

3. This is just the previous branch mutatis mutandis: exchange $\varphi_{2}$ and $\varphi_{3}$ and similarly $\gamma_{2}$ and $\gamma_{3}$.

4. (a) There are no solutions.

(b) There is a one-parameter family of solutions:

$$
\gamma_{1}=\gamma_{2}=\gamma_{3}=\frac{1}{3}, \quad \varphi_{1}=-\frac{1}{3}, \quad \varphi_{2}=\frac{1}{\sqrt{3}} \cos \alpha \quad \text { and } \quad \varphi_{3}=\frac{1}{\sqrt{3}} \sin \alpha .
$$

This generalises two of the solutions mentioned above, to which it reduces when the angle $\alpha$ obeys $2 \alpha \in \pi \mathbb{Z}$. It will be studied in more detail in section 8.2.2.

5. There are no solutions.

6. There are no solutions.

7. This is the previous branch mutatis mutandis, hence there are no solutions.

8. There are no solutions.

In summary, we have found three classes of homogeneous supergravity backgrounds not of AdS type with symmetry group locally isomorphic to $\mathrm{SO}(3,2) \times \mathrm{SO}(5)$, and which we describe in more detail in section 8.2.

\section{Homogeneous anti de Sitter backgrounds}

In this section we study the existence of homogeneous (anti) de Sitter backgrounds $G / H$ with $G$ locally isomorphic to $\mathrm{SO}(n) \times \mathrm{SO}(3,2)$, for $n>4$. Our first result is that there are no de Sitter backgrounds, which allows us to focus on backgrounds of the form $\operatorname{AdS}_{4} \times X^{7}$, where $X=\mathrm{SO}(n) / H$ for $n=5,6,7$. This means that $H$ is a closed Lie subgroup of dimension $3,8,14$, respectively.

One could ask whether there are backgrounds of the type $\operatorname{AdS}_{p} \times X^{11-p}$ for $p \neq 4$ and still of the form $G / H$. This would require $\mathrm{SO}(3,2)$ acting locally transitively on $\mathrm{AdS}_{p}$. By dimension, and since $\mathrm{SO}(3,2)$ must act effectively, $p \geq 4$. One can easily show that $\mathrm{SO}(3,2)$ cannot act locally transitively on $\mathrm{AdS}_{5}$. This is done by comparing the possible subgroups of $\mathrm{SO}(3,2)$ which admit an embedding into $\mathrm{SO}(4,1)$, as listed in [16], and checking that 
the linear isotropy representation of the unique such subgroup (with Lie algebra of type $A_{5,35}^{0,1}$ in that paper's notation) does not in fact lie in $\mathrm{SO}(4,1)$. We do not know whether $\mathrm{AdS}_{p}$ for $p>5$ admits an isometric transitive action of $\mathrm{SO}(3,2)[24]$.

\subsection{There are no de Sitter backgrounds}

It is probably the case that $\mathrm{SO}(3,2)$ does not act isometrically on any de Sitter space, but let us in any case show that the Einstein equations for homogeneous backgrounds rule out a de Sitter solution. Let us consider a geometry of the form $\mathrm{dS}_{p} \times M^{11-d}$. Since the only invariant forms on $\mathrm{dS}_{p}$ are the constant 0 -forms and constant multiples of the volume $p$-form $\nu$, if $p>4 F$ cannot have legs along the de Sitter directions, whence the de Sitter components of the Einstein equation are given by

$$
R_{\mu \nu}=-\frac{1}{6} g_{\mu \nu}|F|^{2} \Longrightarrow g^{\mu \nu} R_{\mu \nu}=-\frac{p}{6}|F|^{2} \leq 0,
$$

contradicting that de Sitter space has positive scalar curvature. If $p \leq 4$, then the most general invariant $F$ has the form

$$
F=\nu \wedge \alpha+\varphi \quad \text { for } \quad \alpha \in \Omega^{4-p}(M), \varphi \in \Omega^{4}(M)
$$

The de Sitter components of the Einstein equation are now given by

$$
R_{\mu \nu}=-\frac{1}{2} g_{\mu \nu}|\alpha|^{2}-\frac{1}{6} g_{\mu \nu}\left(-|\alpha|^{2}+|\varphi|^{2}\right) \Longrightarrow g^{\mu \nu} R_{\mu \nu}=-\frac{p}{6}\left(2|\alpha|^{2}+|\varphi|^{2}\right) \leq 0,
$$

again yielding a contradiction. In summary, there are no homogeneous de Sitter backgrounds.

\subsection{No new $n=7 \quad \mathrm{AdS}_{4}$ backgrounds}

From the results of section 4.6, we see that there is a unique 14-dimensional Lie subalgebra of $\mathfrak{s o}(7)$, namely $\mathfrak{g}_{2}$. The reductive split $\mathfrak{s o}(7)=\mathfrak{g}_{2} \oplus \mathfrak{m}$ is such that $\mathfrak{m}$ is the 7-dimensional irreducible representation of $\mathfrak{g}_{2}$, whence the homogeneous space $\mathrm{SO}(7) / G_{2}$ is locally isometric to the round 7-sphere, which admits an isometric action of $\mathrm{SO}(8)$ with stabiliser $\mathrm{SO}(7)$. Now the only homogeneous background $\mathrm{AdS}_{4} \times \mathrm{SO}(8) / \mathrm{SO}(7)$ is a Freund-Rubin background, because there are no $\mathrm{SO}(8)$-invariant 4-forms on $\mathrm{SO}(8) / \mathrm{SO}(7)$; however, there are $\mathrm{SO}(7)$-invariant 4-forms on $\mathrm{SO}(7) / G_{2}$ and hence in principle one can ask whether there are supergravity backgrounds on $\mathrm{AdS}_{4} \times \mathrm{SO}(7) / G_{2}$ which are not of Freund-Rubin type. Metrically, of course, such backgrounds are locally isometric to $\mathrm{AdS}_{4} \times S^{7}$, but where the radii of curvature of the two spaces are fixed by the flux. Recall that $\mathrm{SO}(7)$-invariant 4-forms on $X=\mathrm{SO}(7) / G_{2}$ are in one-to-one correspondence with $G_{2}$-invariant elements of $\Lambda^{4} \mathfrak{m}$. It is well-known that $\left(\Lambda^{p} \mathfrak{m}\right)^{G_{2}}$ is one-dimensional for $p=3,4$. If we let $\varphi$ denote a nonzero $\mathrm{SO}(7)$-invariant 3 -form, then the $\mathrm{SO}(7)$-invariant 4 -form is proportional to $\star \varphi$. Moreover it is also the case that $d \varphi$ is proportional to $\star \varphi$, whence $d \star \varphi=0$. It follows by dimension that $\star \varphi \wedge \star \varphi=0$, and that $\delta \star \varphi \neq 0$, since it is in fact proportional to $\varphi$. Therefore letting $F=\alpha \operatorname{dvol}_{\mathrm{AdS}_{4}}+\beta \star \varphi$ with $\alpha, \beta \in \mathbb{R}$, we see that $d F=0$ and that both $-\frac{1}{2} \star F \wedge F$ and $\delta F$ are proportional to $\varphi$, whence we get an identity relating $\beta$ and $\alpha \beta$, 
which means that either $\beta=0$ (Freund-Rubin) or else $\alpha$ is fixed and $\beta$ free. The former background is the standard Freund-Rubin background $\mathrm{AdS}_{4} \times S^{7}$, whereas the latter is the Englert solution [25]. It may be worth writing these solutions explicitly in our conventions.

We have $\mathfrak{g}=\mathfrak{s o}(3,2) \oplus \mathfrak{s o}(7)$ with bases $J_{\mu \nu}$ for $\mathfrak{s o}(3,2)$ and $L_{a b}$ for $\mathfrak{s o}(7)$. We have $\mathfrak{h}=\mathfrak{s o}(3,1) \oplus \mathfrak{g}_{2}$, where $\mathfrak{s o}(3,1)$ is spanned by $J_{\mu \nu}$, with $\mu, \nu=1,2,3,4$, and $\mathfrak{g}_{2}$ is spanned by the 14 linear combinations in equation (4.12). This means that an ordered basis $\left(X_{0}, X_{1}, \ldots, X_{\mathfrak{\natural}}\right)$ for the complement of $\mathfrak{h}$ in $\mathfrak{g}$ is given by the following elements of $\mathfrak{g}$ in the order given:

$$
\begin{gathered}
J_{45}, J_{15}, J_{25}, J_{35}, L_{12}+L_{34}-L_{67}, L_{13}-L_{24}+L_{57}, L_{14}+L_{23}-L_{56}, \\
L_{15}-L_{37}+L_{46}, L_{16}+L_{27}-L_{45}, L_{17}-L_{26}+L_{35}, L_{25}+L_{36}+L_{47} .
\end{gathered}
$$

We let $\left(\theta^{0}, \ldots, \theta^{\natural}\right)$ denote the canonical dual basis for $\mathfrak{m}^{*}$. The most general $H$-invariant lorentzian inner product on $\mathfrak{m}$ is given by

$$
g=\gamma_{0}\left(-\left(\theta^{0}\right)^{2}+\left(\theta^{1}\right)^{2}+\left(\theta^{2}\right)^{2}+\left(\theta^{3}\right)^{2}\right)+\gamma_{1} \sum_{i=4}^{\natural}\left(\theta^{i}\right)^{2},
$$

where $\gamma_{0}>0$ and $\gamma_{1}>0$. Similarly, the most general invariant 4 -form on $\mathfrak{m}$ is given by

$$
F=\varphi_{1} \theta^{0123}+\varphi_{2}\left(\theta^{4578}+\theta^{459 \natural}+\theta^{4679}-\theta^{468 \natural}+\theta^{567 \natural}+\theta^{5689}-\theta^{789 \natural}\right),
$$

which is closed for all $\varphi_{1}$ and $\varphi_{2}$. The homothety invariance of the field equations allows us to set $\gamma_{0}=1$, and we will do so. The Maxwell equations (5.7) then become

$$
\varphi_{2}\left(\varphi_{1}+\frac{6}{\sqrt{\gamma_{1}}}\right)=0
$$

which has two branches: one where $\varphi_{2}=0$ and $\varphi_{1}$ remains free, and one where $\varphi_{1}=$ $-6 / \sqrt{\gamma_{1}}$ and $\varphi_{2}$ remains free. The Einstein equations (5.8) become

$$
18 \gamma_{1}^{4}=2 \gamma_{1}^{4} \varphi_{1}^{2}+7 \varphi_{2}^{2} \quad \text { and } \quad 81 \gamma_{1}^{3}=\gamma_{1}^{4} \varphi_{1}^{2}+5 \varphi_{2}^{2}
$$

The branch where $\varphi_{2}=0$ corresponds to the original Freund-Rubin background [5], in which $\varphi_{1}= \pm 3$ and $\gamma_{1}=9$. Reintroducing the scale $\lambda \in \mathbb{R}^{\times}$, we have

$$
\begin{aligned}
& \lambda^{-2} g=-\left(\theta^{0}\right)^{2}+\left(\theta^{1}\right)^{2}+\left(\theta^{2}\right)^{2}+\left(\theta^{3}\right)^{2}+9 \sum_{i=4}^{\natural}\left(\theta^{i}\right)^{2} \\
& \lambda^{-3} F=3 \theta^{0123} .
\end{aligned}
$$

In the second branch, and reintroducing the scale, we have

$$
\begin{aligned}
& \lambda^{-2} g=-\left(\theta^{0}\right)^{2}+\left(\theta^{1}\right)^{2}+\left(\theta^{2}\right)^{2}+\left(\theta^{3}\right)^{2}+\frac{15}{2} \sum_{i=4}^{\natural}\left(\theta^{i}\right)^{2} \\
& \lambda^{-3} F=-2 \sqrt{\frac{6}{5}} \theta^{0123} \pm 3\left(\frac{15}{2}\right)^{3 / 2}\left(\theta^{4578}+\theta^{459 \natural}+\theta^{4679}-\theta^{468 \natural}+\theta^{567 \natural}+\theta^{5689}-\theta^{789 \natural}\right) .
\end{aligned}
$$

This is Englert solution [25], which is known not to be supersymmetric. 


\subsection{No new $n=6 \quad \mathrm{AdS}_{4}$ backgrounds}

As we saw in section 4.5 , there is a unique subalgebra of $\mathfrak{s o}(6)$ of dimension 8 , namely $\mathfrak{s u}(3)$. The reductive split $\mathfrak{s o}(6)=\mathfrak{s u}(3) \oplus \mathfrak{p}$ is such that $\mathfrak{p}$ is a reducible representation of $\mathfrak{s u}(3)$, whose complexification $\mathfrak{p} \otimes_{\mathbb{R}} \mathbb{C}=V_{[00]} \oplus V_{[10]} \oplus V_{[01]}$, where $[\mathrm{mn}]$ are the Dynkin labels of the representations, with [00] corresponding to the trivial one-dimensional representation and $[10]$ and [01] to the fundamental and anti-fundamental three-dimensional representations, respectively. As a real representation, $\mathfrak{p}$ decomposes into the direct sum of a the trivial onedimensional representation and an irreducible six-dimensional real representation whose complexification is $V_{[10]} \oplus V_{[01]}$. This means that there are two parameters for the inner product on $\mathfrak{p}$, which together with the radius of curvature of $\mathrm{AdS}_{4}$ makes three metric parameters. There is a four-dimensional space of invariant 4-forms: the volume form on $\mathrm{AdS}_{4}$, the square of the invariant symplectic form on $\mathfrak{p}$ and two more coming from the 3 -forms on $V_{[10]}$ and on $V_{[01]}$ wedged with any nonzero element of $V_{[00]}^{*}$. Let us be more explicit.

We have $\mathfrak{g}=\mathfrak{s o}(3,2) \oplus \mathfrak{s o}(6)$ with bases $J_{\mu \nu}$ for $\mathfrak{s o}(3,2)$ and $L_{a b}$ for $\mathfrak{s o}(6)$. We have $\mathfrak{h}=\mathfrak{s o}(3,1) \oplus \mathfrak{s u}(3)$, where $\mathfrak{s o}(3,1)$ is spanned by $J_{\mu \nu}$, with $\mu, \nu=1,2,3,4$, and $\mathfrak{s u}(3)$ is spanned by the 8 linear combinations in equation (4.7). This means that an ordered basis $\left(X_{0}, X_{1}, \ldots, X_{\mathfrak{\natural}}\right)$ for the complement of $\mathfrak{h}$ in $\mathfrak{g}$ is given by the following elements of $\mathfrak{g}$ in the order given:

$$
\begin{aligned}
& J_{45}, J_{15}, J_{25}, J_{35}, L_{12}+L_{34}+L_{56}, L_{13}-L_{24}, L_{14}+L_{23}, L_{15}-L_{26}, \\
& L_{16}+L_{25}, L_{35}-L_{46}, L_{36}+L_{45} .
\end{aligned}
$$

We let $\left(\theta^{0}, \ldots, \theta^{\natural}\right)$ denote the canonical dual basis for $\mathfrak{m}^{*}$. The most general $H$-invariant lorentzian inner product on $\mathfrak{m}$ is given by

$$
g=\gamma_{0}\left(-\left(\theta^{0}\right)^{2}+\left(\theta^{1}\right)^{2}+\left(\theta^{2}\right)^{2}+\left(\theta^{3}\right)^{2}\right)+\gamma_{1}\left(\theta^{4}\right)^{2}+\gamma_{2} \sum_{i=5}^{\natural}\left(\theta^{i}\right)^{2},
$$

where $\gamma_{0,1,2}>0$. Similarly, the most general invariant 4 -form on $\mathfrak{m}$ is given by

$$
\begin{aligned}
F= & \varphi_{1} \theta^{0123}+\varphi_{2}\left(\theta^{4579}-\theta^{458 \natural}-\theta^{467 \natural}-\theta^{4689}\right) \\
& +\varphi_{3}\left(\theta^{457 \natural}+\theta^{4589}+\theta^{4679}-\theta^{468 \natural}\right)+\varphi_{4}\left(\theta^{5678}+\theta^{569 \natural}+\theta^{789 \natural}\right),
\end{aligned}
$$

which is closed for all values of $\varphi_{i}$. The homothety invariance of the field equations allows us to set $\gamma_{0}=1$, and we will do so. The Maxwell equations (5.7) then become

$$
\varphi_{2}\left(\frac{6}{\sqrt{\gamma_{1}}}+\varphi_{1}\right)=0 \quad \varphi_{3}\left(\frac{6}{\sqrt{\gamma_{1}}}+\varphi_{1}\right)=0 \quad \varphi_{4}\left(\frac{8 \sqrt{\gamma_{1}}}{3 \gamma_{2}}+\varphi_{1}\right)=0,
$$

which has several branches:

1. $\varphi_{2}=\varphi_{3}=\varphi_{4}=0$;

2. $\varphi_{2}=\varphi_{3}=0, \varphi_{4} \neq 0$ : whence $\varphi_{1}=-\frac{8 \sqrt{\gamma_{1}}}{3 \gamma_{2}}$; 
3. $\varphi_{2}^{2}+\varphi_{3}^{2} \neq 0, \varphi_{4}=0$ : whence $\varphi_{1}=-\frac{6}{\sqrt{\gamma_{1}}}$

4. $\varphi_{2}^{2}+\varphi_{3}^{2} \neq 0, \varphi_{4} \neq 0$ : whence $\varphi_{1}=-\frac{6}{\sqrt{\gamma_{1}}}$ and $\gamma_{2}=4 \gamma_{1} / 9$.

The Einstein equations (5.8) become

$$
\begin{aligned}
& 0=3 \gamma_{1} \gamma_{2}^{4} \varphi_{1}^{2}+6 \gamma_{2} \varphi_{2}^{2}+6 \gamma_{2} \varphi_{3}^{2}+9 \gamma_{1} \varphi_{4}^{2}-144 \gamma_{1} \gamma_{2}^{3}+16 \gamma_{1}^{2} \gamma_{2}^{2} \\
& 0=2 \gamma_{1} \gamma_{2}^{4} \varphi_{1}^{2}+4 \gamma_{2} \varphi_{2}^{2}+4 \gamma_{2} \varphi_{3}^{2}+3 \gamma_{1} \varphi_{4}^{2}-18 \gamma_{1} \gamma_{2}^{4} \\
& 0=\gamma_{1} \gamma_{2}^{4} \varphi_{1}^{2}+8 \gamma_{2} \varphi_{2}^{2}+8 \gamma_{2} \varphi_{3}^{2}-3 \gamma_{1} \varphi_{4}^{2}-16 \gamma_{1}^{2} \gamma_{2}^{2}
\end{aligned}
$$

It is now a simple matter to specialise the Einstein equations to each of the branches of solutions of the Maxwell equations. We find three kinds of solutions: the original FreundRubin solution, the Englert solution and a circle's worth of solutions found by Pope and Warner [26, 27]. In detail, we have the following results for the above four branches of solutions of the Maxwell equations.

1. This is the Freund-Rubin background. The only solutions to the Einstein equations are $\varphi_{1}= \pm 3, \gamma_{1}=9$ and $\gamma_{2}=4$. Reintroducing the scale, we have

$$
\begin{aligned}
& \lambda^{-2} g=-\left(\theta^{0}\right)^{2}+\left(\theta^{1}\right)^{2}+\left(\theta^{2}\right)^{2}+\left(\theta^{3}\right)^{2}+9\left(\theta^{4}\right)^{2}+4 \sum_{i=5}^{\natural}\left(\theta^{i}\right)^{2} \\
& \lambda^{-3} F=3 \theta^{0123} .
\end{aligned}
$$

2. There are no real solutions to the Einstein equations.

3. Here $\varphi_{1}=-\sqrt{3}, \varphi_{2}+i \varphi_{3}=32 \sqrt{\frac{2}{3}} e^{i \alpha}, \gamma_{1}=12$ and $\gamma_{2}=\frac{8}{3}$, where $\alpha$ is an angle. Reintroducing the scale, we have

$$
\begin{aligned}
\lambda^{-2} g= & -\left(\theta^{0}\right)^{2}+\left(\theta^{1}\right)^{2}+\left(\theta^{2}\right)^{2}+\left(\theta^{3}\right)^{2}+12\left(\theta^{4}\right)^{2}+\frac{8}{3} \sum_{i=5}^{\natural}\left(\theta^{i}\right)^{2} \\
\lambda^{-3} F= & -\sqrt{3} \theta^{0123}+32 \sqrt{\frac{2}{3}} \cos \alpha\left(\theta^{4579}-\theta^{458 \natural}-\theta^{467 \natural}-\theta^{4689}\right) \\
& +32 \sqrt{\frac{2}{3}} \sin \alpha\left(\theta^{457 \natural}+\theta^{4589}+\theta^{4679}-\theta^{468 \natural}\right) .
\end{aligned}
$$

This is the solution found by Pope and Warner.

4. Here $\varphi_{1}=-2 \sqrt{\frac{6}{5}}, \varphi_{2}+i \varphi_{3}=10 \sqrt{\frac{10}{3}} e^{i \alpha}, \varphi_{4}= \pm \frac{20}{3} \sqrt{\frac{10}{3}}, \gamma_{1}=\frac{15}{2}$ and $\gamma_{2}=\frac{10}{3}$, where $\alpha$ is again an angle. Reintroducing the scale, we have

$$
\begin{aligned}
\lambda^{-2} g= & -\left(\theta^{0}\right)^{2}+\left(\theta^{1}\right)^{2}+\left(\theta^{2}\right)^{2}+\left(\theta^{3}\right)^{2}+\frac{15}{2}\left(\theta^{4}\right)^{2}+\frac{10}{3} \sum_{i=5}^{\natural}\left(\theta^{i}\right)^{2} \\
\lambda^{-3} F= & -2 \sqrt{\frac{6}{5}} \theta^{0123}+10 \sqrt{\frac{10}{3}} \cos \alpha\left(\theta^{4579}-\theta^{458 \natural}-\theta^{467 \natural}-\theta^{4689}\right) \\
& +10 \sqrt{\frac{10}{3}} \sin \alpha\left(\theta^{457 \natural}+\theta^{4589}+\theta^{4679}-\theta^{468 \natural}\right) \pm \frac{20}{3} \sqrt{\frac{10}{3}}\left(\theta^{5678}+\theta^{569 \natural}+\theta^{789 \natural}\right) .
\end{aligned}
$$


This is again Englert's solution, but in a language where only the $\mathrm{SO}(6)$ symmetry is manifest. This explains the fact that we get a circle of solutions. The normaliser of $\mathrm{SO}(6)$ in $\mathrm{SO}(8)$ contains an $\mathrm{SO}(2)$ subgroup (in fact, in the centraliser) and the circle is nothing but the orbit of this subgroup. Each point in the circle is invariant under a different $\mathrm{SO}(7)$ subgroup of $\mathrm{SO}(8)$ containing the same $\mathrm{SO}(6)$ subgroup. These $\mathrm{SO}(7)$ subgroups are conjugate in $\mathrm{SO}(8)$ under the action of the normaliser of $\mathrm{SO}(6)$. We will see below when discussing $n=5$ backgrounds that we get a 2 -sphere's worth of Englert solutions, where the 2-sphere is the orbit of the centraliser of $\mathrm{SO}(5)$ in $\mathrm{SO}(8)$, which is an $\mathrm{SO}(3)$ subgroup.

\subsection{Possible new $n=5 \mathrm{AdS}_{4}$ backgrounds}

From the results of section 4.4 we have three 3 -dimensional subalgebras of $\mathfrak{s o}(5)$, all isomorphic to $\mathfrak{s o}(3)$. We can distinguish them by what they do to the five-dimensional irreducible representation of $\mathfrak{s o}(5)$. One acts irreducibly, a second $\mathfrak{s o}(3)$ subalgebra decomposes the five-dimensional representation as $2 V_{0} \oplus V_{2}$, where $V_{n}$ is the $(n+1)$-dimensional irreducible representation of $\mathfrak{s o}(3)$, and the third $\mathfrak{s o}(3)$ subalgebra decomposes it as $V_{0} \oplus V_{3}$. If we let $L_{a b}$ denote the standard basis for $\mathfrak{s o}(5)$, then the three $\mathfrak{s o}(3)$ subalgebras are the following:

1. $\mathfrak{s o}(3)_{\text {irr }}$ with basis given by equation (4.4).

2. $\mathfrak{s o}(3)=\mathbb{R}\left\langle L_{12}, L_{13}, L_{23}\right\rangle$

3. $\mathfrak{s o}(3)_{+}=\mathbb{R}\left\langle L_{1}^{+}, L_{2}^{+}, L_{3}^{+}\right\rangle$

\subsection{1 $\mathfrak{s o}(3)_{\text {irr }}$ isotropy}

The first case, where the subalgebra is $\mathfrak{s o}(3)_{\text {irr }}$, is the simplest. The complement of $\mathfrak{s o}(3)_{\text {irr }}$ in $\mathfrak{s o}(5)$ is irreducible, so there is (up to the overall homothety) one metric parameter. There is a two-dimensional space of closed invariant 4 -forms, spanned by the volume form on $\mathrm{AdS}_{4}$ and a 4 -form on the riemannian factor. The supergravity field equations reveal two backgrounds, which can be shown to be the original Freund-Rubin and Englert backgrounds.

\subsection{2 $\mathfrak{s o}(3)$ isotropy}

In the second case, the isotropy subalgebra is $\mathfrak{s o}(3)$, whose complement in $\mathfrak{s o}(5)$ decomposes as $2 V_{2} \oplus V_{0}$, whence there are four metric parameters, which are reduced to three by the action of the normaliser. In particular, we can choose the inner product to be diagonal relative to the following ordered basis for $\mathfrak{m}$ :

$$
\left(X_{0}, X_{1}, \ldots, X_{\natural}\right)=\left(J_{45}, J_{15}, J_{25}, J_{35}, L_{14}, L_{24}, L_{34}, L_{15}, L_{25}, L_{35}, L_{45}\right) \text {. }
$$

Indeed, in terms of the canonical dual bases for $\mathfrak{m}^{*}$, we can write the invariant metric as

$$
g=\gamma_{0}\left(-\left(\theta^{0}\right)^{2}+\left(\theta^{1}\right)^{2}+\left(\theta^{2}\right)^{2}+\left(\theta^{3}\right)^{2}\right)+\gamma_{1} \sum_{i=4}^{6}\left(\theta^{i}\right)^{2}+\gamma_{2} \sum_{i=7}^{9}\left(\theta^{i}\right)^{2}+\gamma_{3}\left(\theta^{\natural}\right)^{2},
$$


with $\gamma_{0,1,2,3}>0$. Using the homothety invariance, we can set $\gamma_{0}=1$ without loss of generality. The space of invariant closed 4 -forms is 6 -dimensional, whence the most general such $F$ is

$$
\begin{aligned}
F= & \varphi_{1} \theta^{0123}+\varphi_{2} \theta^{456 \natural}+\varphi_{3}\left(\theta^{4578}+\theta^{4679}+\theta^{5689}\right)+\varphi_{4}\left(\theta^{459 \natural}-\theta^{468 \natural}+\theta^{567 \natural}\right) \\
& +\varphi_{5}\left(\theta^{489 \natural}-\theta^{579 \natural}+\theta^{678 \natural}\right)+\varphi_{6} \theta^{789 \natural} .
\end{aligned}
$$

The Maxwell equations (5.7) become the following equations

$$
\begin{array}{ll}
0=\frac{\sqrt{\gamma_{2}} \varphi_{1} \varphi_{2}}{\sqrt{\gamma_{1}}+\frac{3 \varphi_{5}}{\sqrt{\gamma_{3}}}} & 0=-\frac{\sqrt{\gamma_{2}} \varphi_{1} \varphi_{4}}{\sqrt{\gamma_{1}}}+\frac{2 \gamma_{2} \varphi_{4}}{\gamma_{1} \sqrt{\gamma_{3}}}-\frac{\gamma_{1} \varphi_{6}}{\gamma_{2} \sqrt{\gamma_{3}}} \\
0=\frac{3 \varphi_{4}}{\sqrt{\gamma_{3}}+\frac{\sqrt{\gamma_{1}} \varphi_{1} \varphi_{6}}{\sqrt{\gamma_{2}}}} & 0=-\frac{\gamma_{2} \varphi_{2}}{\gamma_{1} \sqrt{\gamma_{3}}}-\frac{\sqrt{\gamma_{1}} \varphi_{1} \varphi_{5}}{\sqrt{\gamma_{2}}}+\frac{2 \gamma_{1} \varphi_{5}}{\gamma_{2} \sqrt{\gamma_{3}}} \\
0=\frac{2 \sqrt{\gamma_{3}} \varphi_{3}}{\sqrt{\gamma_{1}}}-\frac{\varphi_{1} \varphi_{3}}{\sqrt{\gamma_{2}}} &
\end{array}
$$

The first two equations on the left allow us to solve for $\varphi_{4,5}$ :

$$
\varphi_{4}=-\frac{\sqrt{\gamma_{1} \gamma_{3}}}{3 \sqrt{\gamma_{2}}} \varphi_{1} \varphi_{6} \quad \text { and } \quad \varphi_{5}=-\frac{\sqrt{\gamma_{2} \gamma_{3}}}{3 \sqrt{\gamma_{1}}} \varphi_{1} \varphi_{2}
$$

Inserting this in the remaining equations we are left with

$$
\begin{aligned}
& 0=\varphi_{3}\left(\varphi_{1}-\frac{2 \sqrt{\gamma_{2} \gamma_{3}}}{\sqrt{\gamma_{1}}}\right) \\
& 0=\varphi_{2}\left(\varphi_{1}^{2}-\frac{2 \sqrt{\gamma_{1}} \varphi_{1}}{\sqrt{\gamma_{2} \gamma_{3}}}-\frac{3 \gamma_{2}}{\gamma_{1} \gamma_{3}}\right) \\
& 0=\varphi_{6}\left(\varphi_{1}^{2}-\frac{2 \sqrt{\gamma_{2}} \varphi_{1}}{\sqrt{\gamma_{1} \gamma_{3}}}-\frac{3 \gamma_{1}}{\gamma_{2} \gamma_{3}}\right),
\end{aligned}
$$

which leads to eight branches depending on whether $\varphi_{2,3,6}$ do or do not vanish. For each such branch we have analysed the Einstein equations (5.8), given by

$$
\begin{aligned}
0= & \varphi_{1} \varphi_{2} \varphi_{6}\left(3\left(\gamma_{1}+\gamma_{2}\right)-2 \sqrt{\gamma_{1} \gamma_{2} \gamma_{3}} \varphi_{1}\right) \\
0= & -3 \gamma_{2}{ }^{3} \gamma_{3} \gamma_{1}{ }^{3} \varphi_{1}{ }^{2}-6 \gamma_{1}{ }^{3} \varphi_{6}{ }^{2}-\gamma_{2}{ }^{2} \gamma_{3} \gamma_{1} \varphi_{1}{ }^{2} \varphi_{2}{ }^{2}-9 \gamma_{2} \gamma_{3} \gamma_{1} \varphi_{3}{ }^{2}+3 \gamma_{2}{ }^{3} \varphi_{2}{ }^{2}-9 \gamma_{2}{ }^{2} \gamma_{1}{ }^{4} \\
& +54 \gamma_{2}{ }^{2} \gamma_{3} \gamma_{1}{ }^{3}+9 \gamma_{2}{ }^{4} \gamma_{1}{ }^{2}-9 \gamma_{2}{ }^{2} \gamma_{3}{ }^{2} \gamma_{1}{ }^{2} \\
0= & -3 \gamma_{2}{ }^{3} \gamma_{3} \gamma_{1}{ }^{3} \varphi_{1}{ }^{2}+3 \gamma_{1}{ }^{3} \varphi_{6}{ }^{2}-\gamma_{2} \gamma_{3} \gamma_{1}{ }^{2} \varphi_{1}{ }^{2} \varphi_{6}{ }^{2}-9 \gamma_{2} \gamma_{3} \gamma_{1} \varphi_{3}{ }^{2}-6 \gamma_{2}{ }^{3} \varphi_{2}{ }^{2}+9 \gamma_{2}{ }^{2} \gamma_{1}{ }^{4} \\
& -9 \gamma_{2}{ }^{4}{\gamma_{1}}^{2}-9 \gamma_{2}{ }^{2} \gamma_{3}{ }^{2} \gamma_{1}{ }^{2}+54 \gamma_{2}{ }^{3} \gamma_{3} \gamma_{1}{ }^{2} \\
0= & -3 \gamma_{2}{ }^{3} \gamma_{3} \gamma_{1}{ }^{3} \varphi_{1}{ }^{2}-6 \gamma_{1}{ }^{3} \varphi_{6}{ }^{2}-2 \gamma_{2} \gamma_{3} \gamma_{1}{ }^{2} \varphi_{1}{ }^{2} \varphi_{6}{ }^{2}-2 \gamma_{2}{ }^{2} \gamma_{3} \gamma_{1} \varphi_{1}{ }^{2} \varphi_{2}{ }^{2}+9 \gamma_{2} \gamma_{3} \gamma_{1} \varphi_{3}{ }^{2} \\
& -6 \gamma_{2}{ }^{3} \varphi_{2}{ }^{2}-27 \gamma_{2}{ }^{2} \gamma_{1}{ }^{4}+54 \gamma_{2}{ }^{3} \gamma_{1}{ }^{3}-27 \gamma_{2}{ }^{4} \gamma_{1}{ }^{2}+27 \gamma_{2}{ }^{2} \gamma_{3}{ }^{2} \gamma_{1}{ }^{2} \\
0= & -6 \gamma_{2}{ }^{3} \gamma_{3} \gamma_{1}{ }^{3} \varphi_{1}{ }^{2}-3 \gamma_{1}{ }^{3} \varphi_{6}{ }^{2}-\gamma_{2} \gamma_{3} \gamma_{1}{ }^{2} \varphi_{1}{ }^{2} \varphi_{6}{ }^{2}-\gamma_{2}{ }^{2} \gamma_{3} \gamma_{1} \varphi_{1}{ }^{2} \varphi_{2}{ }^{2}-9 \gamma_{2} \gamma_{3} \gamma_{1} \varphi_{3}{ }^{2} \\
& -3 \gamma_{2}{ }^{3} \varphi_{2}{ }^{2}+54 \gamma_{2}{ }^{3} \gamma_{3} \gamma_{1}{ }^{3} .
\end{aligned}
$$

The end result is that beyond a known Freund-Rubin background $\mathrm{AdS}_{4} \times V_{2}\left(\mathbb{R}^{5}\right)$ and the Pope-Warner background, we obtain the following backgrounds, where we have reintroduced the scale: 
1. With $\sigma$ a sign, we have

$$
\begin{aligned}
& \lambda^{-2} g=-\left(\theta^{0}\right)^{2}+\left(\theta^{1}\right)^{2}+\left(\theta^{2}\right)^{2}+\left(\theta^{3}\right)^{2}+\frac{39-\sigma \sqrt{201}}{22} \sum_{i=4}^{6}\left(\theta^{i}\right)^{2}+\frac{27+\sigma \sqrt{201}}{22} \sum_{i=7}^{9}\left(\theta^{i}\right)^{2}+\left(\theta^{\natural}\right)^{2}, \\
& \lambda^{-3} F=\sqrt{\frac{19+\sigma \sqrt{201}}{5}} \theta^{0123} \pm \frac{2 \sqrt{15(2155-\sigma 31 \sqrt{201})}}{121}\left(\theta^{4578}+\theta^{4679}+\theta^{5689}\right) ;
\end{aligned}
$$

2. A background we can only approximate numerically: $\varphi_{2}=\varphi_{3}=\varphi_{5}=0$, together with

$$
\begin{array}{ll}
\varphi_{1}=-1.3538010207764224 & \gamma_{1}=2.0506059513936354 \\
\varphi_{4}= \pm 4.562584323795499 & \gamma_{2}=0.5588242551644832 \\
\varphi_{6}= \pm 2.51893274180765 & \gamma_{3}=4.390505589439397
\end{array}
$$

and another background obtained from this by $\varphi_{6} \leftrightarrow \varphi_{2}, \varphi_{4} \leftrightarrow \varphi_{5}$ and $\gamma_{1} \leftrightarrow \gamma_{2}$.

3. A circle's worth of backgrounds with metric

$$
\lambda^{-2} g=-\left(\theta^{0}\right)^{2}+\left(\theta^{1}\right)^{2}+\left(\theta^{2}\right)^{2}+\left(\theta^{3}\right)^{2}+\frac{3+\sqrt{6}}{4} \sum_{i=4}^{9}\left(\theta^{i}\right)^{2}+\frac{3}{2}\left(\theta^{\natural}\right)^{2},
$$

and 4-form

$$
\begin{aligned}
\lambda^{-3} F= & \sqrt{6} \theta^{0123} \pm \frac{3}{8} \sqrt{\frac{29+12 \sqrt{6}}{2}}\left(\theta^{4578}+\theta^{4679}+\theta^{5689}\right) \\
& +\frac{3 \sqrt{3(3+\sqrt{6})}}{8}\left(\cos \alpha\left(\theta^{456 \natural}-\theta^{489 \natural}+\theta^{579 \natural}-\theta^{678 \natural}\right)\right. \\
& \left.+\sin \alpha\left(\theta^{789 \natural}-\theta^{459 \natural}+\theta^{468 \natural}-\theta^{567 \natural}\right)\right),
\end{aligned}
$$

with $\alpha$ an angle. We can write $F$ in a more transparent way as follows:

$$
\begin{aligned}
\lambda^{-3} F= & \sqrt{6} \theta^{0123} \mp \frac{3}{16} \sqrt{\frac{29+12 \sqrt{6}}{2}}\left(\theta^{47}+\theta^{58}+\theta^{69}\right)^{\wedge 2} \\
& +\frac{3 \sqrt{3(3+\sqrt{6})}}{8} \operatorname{Re}\left(e^{i \alpha}\left(\theta^{4}+i \theta^{7}\right) \wedge\left(\theta^{5}+i \theta^{8}\right) \wedge\left(\theta^{6}+i \theta^{9}\right)\right) \wedge \theta^{\natural},
\end{aligned}
$$

where we recognise the transverse Kähler calibration $\theta^{47}+\theta^{58}+\theta^{69}$ and the transverse special lagrangian calibration $\operatorname{Re}\left(e^{i \alpha}\left(\theta^{4}+i \theta^{7}\right) \wedge\left(\theta^{5}+i \theta^{8}\right) \wedge\left(\theta^{6}+i \theta^{9}\right)\right)$.

This background is obtained from the Freund-Rubin background $\mathrm{AdS}_{4} \times V_{2}\left(\mathbb{R}^{5}\right)([28]$, appendix C) by the Englert procedure of constructing a 4-form out of the Killing spinors $[25,29]$. The angle $\alpha$ parametrises the choice of the two Killing spinors out of which we make up the part of $F$ with no legs along $\mathrm{AdS}_{4}$. The background $\mathrm{AdS}_{4} \times V_{2}\left(\mathbb{R}^{5}\right)$ has $\mathrm{SU}(3)$ holonomy. This means that $V_{2}\left(\mathbb{R}^{5}\right)$ admits a twodimensional space of real Killing spinors. Depending on which two spinors we pick, we can construct a Kähler calibration and one of the circle's worth of special lagrangian calibrations on the codimension-one subbundle of the tangent bundle whose 
fibre at the origin is spanned by $X_{4}, \ldots, X_{9}$. In addition, the tangent representation of $\mathrm{SU}(3)$ leaves invariant one direction, which is spanned by $X_{\natural}$ at the origin. We now recognise the second term of $F$ in the expression (7.30) as the square of the transverse Kähler calibration (itself a calibration) and the third term as one of the transverse special lagrangian calibrations wedged with the invariant form $\theta^{\natural}$. This solution is also mentioned in ([28], appendix C) but not given explicitly. As usual in the Englert procedure, supersymmetry is broken. The form of the solution suggests that we should be able to obtain it as well via the Pope-Warner procedure in [27], but we have not tried to do this.

The first two backgrounds seem to be new.

\subsection{3 $\mathfrak{s o}(3)_{+}$isotropy}

In the final case, the isotropy algebra is $\mathfrak{s o}(3)_{+}$, whose complement in $\mathfrak{s o}(5)$ decomposes into

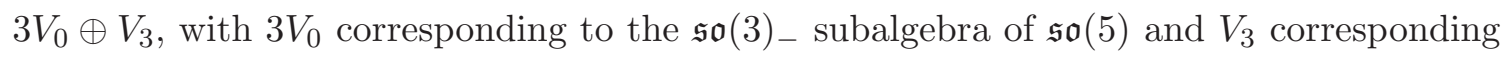
to the four-dimensional representation spanned by the $L_{a 5}$ with $a=1,2,3,4$. This would seem to require 8 parameters to describe the metric, but in fact we can use the action of the normaliser $\mathrm{SO}(3)_{-}$in order to diagonalise the inner product. Indeed, defining the following ordered basis for $\mathfrak{m}$ :

$$
\left(X_{0}, X_{1}, \ldots, X_{\natural}\right)=\left(J_{45}, J_{15}, J_{25}, J_{35}, L_{1}^{-}, L_{2}^{-}, L_{3}^{-}, L_{15}, L_{25}, L_{35}, L_{45}\right),
$$

and the canonical dual bases for $\mathfrak{m}^{*}$, we can write the invariant metric as

$$
g=\gamma_{0}\left(-\left(\theta^{0}\right)^{2}+\left(\theta^{1}\right)^{2}+\left(\theta^{2}\right)^{2}+\left(\theta^{3}\right)^{2}\right)+\gamma_{1}\left(\theta^{4}\right)^{2}+\gamma_{2}\left(\theta^{5}\right)^{2}+\gamma_{3}\left(\theta^{6}\right)^{2}+\gamma_{4} \sum_{i=7}^{\natural}\left(\theta^{i}\right)^{2},
$$

with $\gamma_{0,1,2,3,4}>0$. Using the homothety invariance, we can set $\gamma_{0}=1$ without loss of generality. The space of invariant closed 4-forms is 8-dimensional, with the most general such $F$ given by

$$
\begin{aligned}
F= & \varphi_{1} \theta^{0123}+\varphi_{2}\left(\theta^{4578}-\theta^{459 \natural}\right)+\varphi_{3}\left(\theta^{4579}+\theta^{458 \natural}+\theta^{4678}-\theta^{469 \natural}\right) \\
& +\varphi_{4}\left(\theta^{457 \natural}-\theta^{4589}-\theta^{5678}+\theta^{569 \natural}\right)+\varphi_{5}\left(\theta^{4679}+\theta^{468 \natural}\right) \\
& +\varphi_{6}\left(\theta^{467 \natural}-\theta^{4689}-\theta^{5679}-\theta^{568 \natural}\right)+\varphi_{7}\left(\theta^{567 \natural}-\theta^{5689}\right)+\varphi_{8} \theta^{789 \natural} .
\end{aligned}
$$

The Maxwell equations (5.7) are the following:

$$
\begin{aligned}
& 0=\varphi_{4}\left(\sqrt{\gamma_{1} \gamma_{2} \gamma_{3}} \varphi_{1}+\gamma_{1}+\gamma_{3}\right) \\
& 0=\varphi_{6}\left(\sqrt{\gamma_{1} \gamma_{2} \gamma_{3}} \varphi_{1}+\gamma_{1}+\gamma_{2}\right) \\
& 0=\varphi_{3}\left(\sqrt{\gamma_{1} \gamma_{2} \gamma_{3}} \varphi_{1}+\gamma_{2}+\gamma_{3}\right) \\
& 0=\gamma_{3} \varphi_{2}+\gamma_{2} \varphi_{5}+\gamma_{1} \varphi_{7}+\sqrt{\gamma_{1} \gamma_{2} \gamma_{3}} \varphi_{1} \varphi_{7}+\gamma_{1} \gamma_{2} \gamma_{3} \gamma_{4}^{-2} \varphi_{8} \\
& 0=\gamma_{3} \varphi_{2}-\gamma_{2} \varphi_{5}-\gamma_{1} \varphi_{7}-\sqrt{\gamma_{1} \gamma_{2} \gamma_{3}} \varphi_{1} \varphi_{5}+\gamma_{1} \gamma_{2} \gamma_{3} \gamma_{4}^{-2} \varphi_{8} \\
& 0=\gamma_{3} \varphi_{2}-\gamma_{2} \varphi_{5}+\gamma_{1} \varphi_{7}+\sqrt{\gamma_{1} \gamma_{2} \gamma_{3}} \varphi_{1} \varphi_{2}-\gamma_{1} \gamma_{2} \gamma_{3} \gamma_{4}^{-2} \varphi_{8} \\
& 0=\gamma_{3} \varphi_{2}+\gamma_{2} \varphi_{5}-\gamma_{1} \varphi_{7}-\frac{1}{2} \sqrt{\gamma_{1} \gamma_{2} \gamma_{3}} \varphi_{1} \varphi_{8}
\end{aligned}
$$


The Einstein equations are

$$
\begin{aligned}
0= & \left(\varphi_{2}+\varphi_{5}\right) \varphi_{3}+\varphi_{4} \varphi_{6} \\
0= & \varphi_{3} \varphi_{4}+\left(\varphi_{5}-\varphi_{7}\right) \varphi_{6} \\
0= & \left(\varphi_{2}-\varphi_{7}\right) \varphi_{4}+\varphi_{3} \varphi_{6} \\
9= & \varphi_{1}^{2}+\frac{\varphi_{8}^{2}}{2 \gamma_{4}^{4}}+\frac{\gamma_{3} \varphi_{2}^{2}+\gamma_{2} \varphi_{5}^{2}+\gamma_{1} \varphi_{7}^{2}+\left(\gamma_{2}+\gamma_{3}\right) \varphi_{3}^{2}+\left(\gamma_{1}+\gamma_{3}\right) \varphi_{4}^{2}+\left(\gamma_{1}+\gamma_{2}\right) \varphi_{6}^{2}}{\gamma_{1} \gamma_{2} \gamma_{3} \gamma_{4}^{2}} \\
3\left(\frac{2}{\gamma_{4}}-1\right)= & \frac{\varphi_{8}^{2}}{2 \gamma_{4}^{4}}+\frac{\left(\gamma_{1}+\gamma_{2}+\gamma_{3}\right)}{\gamma_{4}^{2}} \\
0= & \frac{\gamma_{1}^{2}}{2 \gamma_{2} \gamma_{3}}+\frac{\gamma_{1}^{2}}{\gamma_{4}^{2}}-\frac{1}{6} \varphi_{1}^{2} \gamma_{1}+\frac{\varphi_{4}^{2} \gamma_{1}}{3 \gamma_{2} \gamma_{3} \gamma_{4}^{2}}+\frac{\varphi_{6}^{2} \gamma_{1}}{3 \gamma_{2} \gamma_{3} \gamma_{4}^{2}}+\frac{\varphi_{7}^{2} \gamma_{1}}{3 \gamma_{2} \gamma_{3} \gamma_{4}^{2}}+\frac{\varphi_{8}^{2} \gamma_{1}}{6 \gamma_{4}^{4}}-\frac{2 \varphi_{2}^{2}}{3 \gamma_{2} \gamma_{4}^{2}} \\
& -\frac{2 \varphi_{3}^{2}}{3 \gamma_{2} \gamma_{4}^{2}}-\frac{2 \varphi_{3}^{2}}{3 \gamma_{3} \gamma_{4}^{2}}-\frac{2 \varphi_{4}^{2}}{3 \gamma_{2} \gamma_{4}^{2}}-\frac{2 \varphi_{5}^{2}}{3 \gamma_{3} \gamma_{4}^{2}}-\frac{2 \varphi_{6}^{2}}{3 \gamma_{3} \gamma_{4}^{2}}-\frac{\gamma_{3}}{2 \gamma_{2}}-\frac{\gamma_{2}}{2 \gamma_{3}}+1 \\
0= & \frac{\gamma_{2}^{2}}{2 \gamma_{1} \gamma_{3}}+\frac{\gamma_{2}^{2}}{\gamma_{4}^{2}}-\frac{1}{6} \varphi_{1}^{2} \gamma_{2}+\frac{\varphi_{3}^{2} \gamma_{2}}{3 \gamma_{1} \gamma_{3} \gamma_{4}^{2}}+\frac{\varphi_{5}^{2} \gamma_{2}}{3 \gamma_{1} \gamma_{3} \gamma_{4}^{2}}+\frac{\varphi_{6}^{2} \gamma_{2}}{3 \gamma_{1} \gamma_{3} \gamma_{4}^{2}}+\frac{\varphi_{8}^{2} \gamma_{2}}{6 \gamma_{4}^{4}}-\frac{2 \varphi_{2}^{2}}{3 \gamma_{1} \gamma_{4}^{2}} \\
& -\frac{2 \varphi_{3}^{2}}{3 \gamma_{1} \gamma_{4}^{2}}-\frac{2 \varphi_{4}^{2}}{3 \gamma_{1} \gamma_{4}^{2}}-\frac{2 \varphi_{4}^{2}}{3 \gamma_{3} \gamma_{4}^{2}}-\frac{2 \varphi_{6}^{2}}{3 \gamma_{3} \gamma_{4}^{2}}-\frac{2 \varphi_{7}^{2}}{3 \gamma_{3} \gamma_{4}^{2}}-\frac{\gamma_{3}}{2 \gamma_{1}}-\frac{\gamma_{1}}{2 \gamma_{3}}+1 \\
0= & \frac{\gamma_{3}^{2}}{2 \gamma_{1} \gamma_{2}}+\frac{\gamma_{3}^{2}}{\gamma_{4}^{2}}-\frac{1}{6} \varphi_{1}^{2} \gamma_{3}+\frac{\varphi_{2}^{2} \gamma_{3}}{3 \gamma_{1} \gamma_{2} \gamma_{4}^{2}}+\frac{\varphi_{3}^{2} \gamma_{3}}{3 \gamma_{1} \gamma_{2} \gamma_{4}^{2}}+\frac{\varphi_{4}^{2} \gamma_{3}}{3 \gamma_{1} \gamma_{2} \gamma_{4}^{2}}+\frac{\varphi_{8}^{2} \gamma_{3}}{6 \gamma_{4}^{4}}-\frac{2 \varphi_{3}^{2}}{3 \gamma_{1} \gamma_{4}^{2}} \\
& -\frac{2 \varphi_{4}^{2}}{3 \gamma_{2} \gamma_{4}^{2}}-\frac{2 \varphi_{5}^{2}}{3 \gamma_{1} \gamma_{4}^{2}}-\frac{2 \varphi_{6}^{2}}{3 \gamma_{1} \gamma_{4}^{2}}-\frac{2 \varphi_{6}^{2}}{3 \gamma_{2} \gamma_{4}^{2}}-\frac{2 \varphi_{7}^{2}}{3 \gamma_{2} \gamma_{4}^{2}}-\frac{\gamma_{2}}{2 \gamma_{1}}-\frac{\gamma_{1}}{2 \gamma_{2}}+1
\end{aligned}
$$

First of all, let us remark that the equations have a symmetry of order 3 which fixes $\gamma_{4}, \varphi_{1}, \varphi_{8}$ and transforms the remaining parameters as

$$
\left(\gamma_{1}, \gamma_{2}, \gamma_{3}, \varphi_{2}, \varphi_{3}, \varphi_{4}, \varphi_{5}, \varphi_{6}, \varphi_{7}\right) \mapsto\left(\gamma_{2}, \gamma_{3}, \gamma_{1},-\varphi_{7}, \varphi_{4}, \varphi_{6}, \varphi_{2}, \varphi_{3},-\varphi_{5}\right) .
$$

The Einstein equations allow us to solve for $\varphi_{1}$ and $\varphi_{8}$ and one sees quickly that $\gamma_{4}<2$. The Maxwell equations are then linear equations on the remaining $\varphi_{2,3,4,5,6,7}$. The generic solution sets them all to zero, but then this sets $\varphi_{8}=0$ as well. There are two solutions, both of which have $\varphi_{1}= \pm 3$ and $\gamma_{1}=\gamma_{2}=\gamma_{3}$. In one solution we have $\gamma_{1}=\gamma_{2}=\gamma_{3}=$ $\gamma_{4}=1$, which corresponds to the original Freund-Rubin background, whereas in the other case we have $\gamma_{1}=\gamma_{2}=\gamma_{3}=\frac{25}{9}$ and $\gamma_{4}=\frac{5}{9}$, which is the squashed 7 -sphere solution of [30]. Both of these backgrounds have $\gamma_{1}=\gamma_{2}=\gamma_{3}$, but there are others in this class. In fact, one finds a 2-sphere's worth of Englert solutions as well as the squashed Englert solution of [31-33]. In our conventions, the squashed Englert solution looks like

$$
\begin{aligned}
& \lambda^{-2} g=-\left(\theta^{0}\right)^{2}+\left(\theta^{1}\right)^{2}+\left(\theta^{2}\right)^{2}+\left(\theta^{3}\right)^{2}+\frac{3}{10} \sum_{i=4}^{6}\left(\theta^{i}\right)^{2}+\frac{3}{2} \sum_{i=7}^{\natural}\left(\theta^{i}\right)^{2} \\
& \lambda^{-3} F=2 \sqrt{\frac{6}{5}} \theta^{0123} \pm 3\left(\frac{3}{10}\right)^{3 / 2}\left(\theta^{4578}-\theta^{459 \natural}+\theta^{4679}+\theta^{468 \natural}-\theta^{567 \natural}+\theta^{5689}+5 \theta^{789 \natural}\right)
\end{aligned}
$$

where we recognise the second term in $F$ as the $G_{2}$-invariant coassociative calibration built out of one of the Killing spinors of the Freund-Rubin background. 
It remains to look at the cases where the $\gamma_{1}, \gamma_{2}, \gamma_{3}$ are not all equal. If all of $\varphi_{3,4,6}$ are different from zero, then the Maxwell equations show that $\gamma_{1}=\gamma_{2}=\gamma_{3}$, hence we must have at least one of $\varphi_{2,3,6}$ equal to zero. Due to the order-3 symmetry (7.35) we can take $\varphi_{3}=0$ without loss of generality, but then the first of the Einstein equations say that $\varphi_{4} \varphi_{6}=0$ and whence we must have at least two of $\varphi_{2,3,6}$ equal to zero. This gives two cases to consider. In the first case, $\varphi_{3}=\varphi_{4}=0$ and $\varphi_{6} \neq 0$, whereas in the second case $\varphi_{3}=\varphi_{4}=\varphi_{6}=0$.

Let us consider the first case, with $\varphi_{3}=\varphi_{4}=0$ but $\varphi_{6} \neq 0$. This last condition has two immediate consequences: the second Maxwell equation allows us to solve for $\varphi_{1}$ :

$$
\varphi_{1}=-\frac{\gamma_{1}+\gamma_{2}}{\sqrt{\gamma_{1} \gamma_{2} \gamma_{3}}}
$$

whereas the second Einstein equation forces $\varphi_{7}=\varphi_{5}$. The fourth Maxwell equation allows us to solve for $\varphi_{2}$ in terms of $\varphi_{8}$ :

$$
\varphi_{2}=-\frac{\gamma_{1} \gamma_{2}}{\gamma_{4}^{2}} \varphi_{8}
$$

and the fifth Einstein equation allows us to solve for $\varphi_{8}$ up to a sign:

$$
\varphi_{8}= \pm \sqrt{12 \gamma_{4}^{3}-6 \gamma_{4}^{4}-2\left(\gamma_{1}+\gamma_{2}+\gamma_{3}\right) \gamma_{4}^{2}},
$$

whence

$$
\varphi_{2}=\mp \gamma_{1} \gamma_{2} \sqrt{12 \gamma_{4}^{-1}-6-2\left(\gamma_{1}+\gamma_{2}+\gamma_{3}\right) \gamma_{4}^{-2}} .
$$

We now have to distinguish two cases, according to whether or not $\gamma_{1}=\gamma_{2}$. If $\gamma_{1} \neq \gamma_{2}$, we find that there are no admissible solutions to the equations, whereas if $\gamma_{1}=\gamma_{2}$, we get precisely one admissible background:

$$
\begin{aligned}
\lambda^{-2} g= & -\left(\theta^{0}\right)^{2}+\left(\theta^{1}\right)^{2}+\left(\theta^{2}\right)^{2}+\left(\theta^{3}\right)^{2}+\frac{2}{3}\left(\left(\theta^{4}\right)^{2}+\left(\theta^{5}\right)^{2}+2\left(\theta^{6}\right)^{2}\right)+\frac{2}{3} \sum_{i=7}^{\natural}\left(\theta^{i}\right)^{2} \\
\lambda^{-3} F= & -\sqrt{3} \theta^{0123}+2\left(\frac{2}{3}\right)^{3 / 2} \cos \alpha\left(\theta^{4679}+\theta^{468 \natural}+\theta^{567 \natural}-\theta^{5689}\right) \\
& +2\left(\frac{2}{3}\right)^{3 / 2} \sin \alpha\left(\theta^{467 \natural}-\theta^{4689}-\theta^{5679}-\theta^{568 \natural}\right),
\end{aligned}
$$

which is seen to be the Pope-Warner solution.

Finally, it remains to analyse the case where $\varphi_{3}=\varphi_{4}=\varphi_{6}=0$, but yet $\gamma_{1,2,3}$ are not all the same. It is not difficult to solve for $\varphi_{i}^{2}$ using the Einstein equations, since these equations are linear in these variables. One finds

$$
\begin{aligned}
\varphi_{1}^{2} & =\frac{2\left(\gamma_{1}+\gamma_{2}+\gamma_{3}+15 \gamma_{4}^{2}-12 \gamma_{4}\right)}{\gamma_{4}^{2}}+\frac{2\left(\gamma_{1}^{2}+\gamma_{2}^{2}+\gamma_{3}^{2}\right)-\left(\gamma_{1}+\gamma_{2}+\gamma_{3}\right)^{2}}{\gamma_{1} \gamma_{2} \gamma_{3}} \\
\varphi_{2}^{2} & =6 \gamma_{1} \gamma_{2} \gamma_{4}\left(1-\gamma_{4}\right)+\gamma_{1} \gamma_{4}^{2}+\gamma_{2} \gamma_{4}^{2}-\gamma_{3} \gamma_{4}^{2}-\gamma_{1} \gamma_{2} \gamma_{3} \\
\varphi_{5}^{2} & =6 \gamma_{1} \gamma_{3} \gamma_{4}\left(1-\gamma_{4}\right)+\gamma_{1} \gamma_{4}^{2}-\gamma_{2} \gamma_{4}^{2}+\gamma_{3} \gamma_{4}^{2}-\gamma_{1} \gamma_{2} \gamma_{3} \\
\varphi_{7}^{2} & =6 \gamma_{2} \gamma_{3} \gamma_{4}\left(1-\gamma_{4}\right)-\gamma_{1} \gamma_{4}^{2}+\gamma_{2} \gamma_{4}^{2}+\gamma_{3} \gamma_{4}^{2}-\gamma_{1} \gamma_{2} \gamma_{3} \\
\varphi_{8}^{2} & =2\left(3 \gamma_{4}\left(2-\gamma_{4}\right)-\left(\gamma_{1}+\gamma_{2}+\gamma_{3}\right)\right) \gamma_{4}^{2}
\end{aligned}
$$


It follows easily from the last of these equations that $\gamma_{4}<2$ and that $\gamma_{1}+\gamma_{2}+\gamma_{3} \leq 3$, with the bound attained for $\gamma_{4}=1$ and $\varphi_{8}=0$. Since now all $\gamma_{1,2,3}$ are the same, we may exploit the order-3 symmetry (7.35) of the equations in order to make a choice that $\gamma_{1} \neq \gamma_{3}$ and $\gamma_{2} \neq \gamma_{3}$. This leaves open the possibility that $\gamma_{1}$ and $\gamma_{2}$ may be equal.

Alas, we have been unable to solve this system at the present time. We know that there is a Freund-Rubin solution, discovered in [28], and known to possess $N=1$ supersymmetry and therefore also an associated Englert-like background with the four-form $F$ constructed out of the Killing spinor. We expect that there are others, since preliminary numerical investigations suggest that there should be a positive-dimensional moduli space of solutions of these equations.

In summary, we find a number of novel $n=5$ AdS backgrounds whose geometry is studied in more detail in the following section.

\section{The geometry of some $n=5$ backgrounds}

In this section we discuss the geometry of the non-numerical $n=5$ backgrounds we discovered in sections 6.3 and 7.4.

\section{1 $n=5$ anti de Sitter backgrounds}

In section 7.4, we exhibited a number of new (to us) homogeneous $\mathrm{AdS}_{4}$ backgrounds with isometry Lie algebra $\mathfrak{s o}(3,2) \oplus \mathfrak{s o}(5)$. In all cases the geometry is $\mathrm{AdS}_{4} \times P^{7}$, where $P$ is a riemannian manifold homogeneous under the action of $\mathrm{SO}(5)$. The backgrounds in section 7.4.2, whose geometry is given by equations (7.26), (7.27) and (7.28), all have metric of the general form given by equation (7.20) with $\gamma_{0,1,2,3}>0$. Using the homothety invariance of the supergravity field equations, we can set $\gamma_{0}=1$ without much loss of generality. This fixes the scale of the $\mathrm{AdS}_{4}$ factor and the remaining metric freedom resides in the riemannian factor $P^{7}$ with a metric depending on $\gamma_{1,2,3}$. This metric is Einstein when $\gamma_{1}=\gamma_{2}=\frac{2}{3} \gamma_{3}$. In that case, the Einstein condition is $R_{a b}=\lambda g_{a b}$ with $\lambda=\frac{9}{4 \gamma_{2}}$. For those values of the parameters the supergravity field equations are not satisfied, which is to be expected, since the four-form (7.29) is not of Freund-Rubin type.

Let us now discuss the isometries of this family of geometries. Using the method described in appendix $\mathrm{A}$ it is possible to show that the isometry Lie algebra of the general metric (7.20) with $\gamma_{0,1,2,3}>0$ is generically indeed $\mathfrak{s o}(3,2) \oplus \mathfrak{s o}(5)$, but if $\gamma_{1}=\gamma_{2}$ it is enhanced to $\mathfrak{s o}(3,2) \oplus \mathfrak{s o}(5) \oplus \mathfrak{s o}(2)$. This is the case for the background described by equations (7.28) and (7.29) (or (7.30)). The extra Killing vector $\chi$, spans the centre of the isometry Lie algebra and, like any Killing vector, is uniquely defined by its value and that of its derivative at the origin. In the notation of section 7.4.2,

$$
\left.\chi\right|_{o}=\frac{2 \gamma_{1}}{\gamma_{3}} L_{45},\left.\quad \nabla_{L_{i 4}} \chi\right|_{o}=L_{i 5} \quad \text { and }\left.\quad \nabla_{L_{i 5}} \chi\right|_{o}=-L_{i 4},
$$

where $i=1,2,3$ and all other derivatives vanish at the origin. From now on we will take $\gamma_{1}=\gamma_{2}$.

Let us characterise Killing vector fields by pairs $K=(\xi, \Phi)$ where $\xi \in \mathfrak{m}$ is the value of the Killing vector field at the origin and $\Phi=-\nabla \xi \in \mathfrak{s o}(\mathfrak{m})$ is (minus) its derivative at 
the origin. Let $\left(e_{1}, \ldots, e_{7}\right)=\left(L_{14}, L_{24}, L_{34}, L_{15}, L_{25}, L_{35}, L_{45}\right)$ be an ordered basis for $\mathfrak{m}$. A basis for the isometry Lie algebra of the metric on $P$ with the choice $\gamma_{1}=\gamma_{2}$ is given by $K_{a}=\left(\xi_{a}, \Phi_{a}\right)$ for $a=1, \ldots, 11$, where

$$
\xi_{1}=\xi_{2}=\xi_{3}=\xi_{4}=0 \quad \text { and } \quad\left(\xi_{5}, \ldots, \xi_{11}\right)=\left(e_{1}, \ldots, e_{7}\right),
$$

and

$$
\begin{aligned}
& \Phi_{1}=\left(\begin{array}{ccccccc}
0 & 1 & 0 & 0 & 0 & 0 & 0 \\
-1 & 0 & 0 & 0 & 0 & 0 & 0 \\
0 & 0 & 0 & 0 & 0 & 0 & 0 \\
0 & 0 & 0 & 0 & 1 & 0 & 0 \\
0 & 0 & 0 & -1 & 0 & 0 & 0 \\
0 & 0 & 0 & 0 & 0 & 0 & 0 \\
0 & 0 & 0 & 0 & 0 & 0 & 0
\end{array}\right) \quad \Phi_{2}=\left(\begin{array}{ccccccc}
0 & 0 & 1 & 0 & 0 & 0 & 0 \\
0 & 0 & 0 & 0 & 0 & 0 & 0 \\
-1 & 0 & 0 & 0 & 0 & 0 & 0 \\
0 & 0 & 0 & 0 & 0 & 1 & 0 \\
0 & 0 & 0 & 0 & 0 & 0 & 0 \\
0 & 0 & 0 & -1 & 0 & 0 & 0 \\
0 & 0 & 0 & 0 & 0 & 0 & 0
\end{array}\right) \\
& \Phi_{3}=\left(\begin{array}{ccccccc}
0 & 0 & 0 & 1 & 0 & 0 & 0 \\
0 & 0 & 0 & 0 & 1 & 0 & 0 \\
0 & 0 & 0 & 0 & 0 & 1 & 0 \\
-1 & 0 & 0 & 0 & 0 & 0 & 0 \\
0 & -1 & 0 & 0 & 0 & 0 & 0 \\
0 & 0 & -1 & 0 & 0 & 0 & 0 \\
0 & 0 & 0 & 0 & 0 & 0 & 0
\end{array}\right) \quad \Phi_{4}=\left(\begin{array}{ccccccc}
0 & 0 & 0 & 0 & 0 & 0 & 0 \\
0 & 0 & 1 & 0 & 0 & 0 & 0 \\
0 & -1 & 0 & 0 & 0 & 0 & 0 \\
0 & 0 & 0 & 0 & 0 & 0 & 0 \\
0 & 0 & 0 & 0 & 0 & 1 & 0 \\
0 & 0 & 0 & 0 & -1 & 0 & 0 \\
0 & 0 & 0 & 0 & 0 & 0 & 0
\end{array}\right) \\
& \Phi_{5}=\left(\begin{array}{ccccccc}
0 & 0 & 0 & 0 & 0 & 0 & 0 \\
0 & 0 & 0 & 0 & 0 & 0 & 0 \\
0 & 0 & 0 & 0 & 0 & 0 & 0 \\
0 & 0 & 0 & 0 & 0 & 0 & -\frac{\gamma_{3}}{2 \gamma_{1}} \\
0 & 0 & 0 & 0 & 0 & 0 & 0 \\
0 & 0 & 0 & 0 & 0 & 0 & 0 \\
0 & 0 & 0 & \frac{1}{2} & 0 & 0 & 0
\end{array}\right) \quad \Phi_{6}=\left(\begin{array}{ccccccc}
0 & 0 & 0 & 0 & 0 & 0 & 0 \\
0 & 0 & 0 & 0 & 0 & 0 & 0 \\
0 & 0 & 0 & 0 & 0 & 0 & 0 \\
0 & 0 & 0 & 0 & 0 & 0 & 0 \\
0 & 0 & 0 & 0 & 0 & 0 & -\frac{\gamma_{3}}{2 \gamma_{1}} \\
0 & 0 & 0 & 0 & 0 & 0 & 0 \\
0 & 0 & 0 & 0 & \frac{1}{2} & 0 & 0
\end{array}\right) \\
& \Phi_{7}=\left(\begin{array}{ccccccc}
0 & 0 & 0 & 0 & 0 & 0 & 0 \\
0 & 0 & 0 & 0 & 0 & 0 & 0 \\
0 & 0 & 0 & 0 & 0 & 0 & 0 \\
0 & 0 & 0 & 0 & 0 & 0 & 0 \\
0 & 0 & 0 & 0 & 0 & 0 & 0 \\
0 & 0 & 0 & 0 & 0 & 0 & -\frac{\gamma_{3}}{2 \gamma_{1}} \\
0 & 0 & 0 & 0 & 0 & \frac{1}{2} & 0
\end{array}\right) \quad \Phi_{8}=\left(\begin{array}{ccccccc}
0 & 0 & 0 & 0 & 0 & 0 & \frac{\gamma_{3}}{2 \gamma_{1}} \\
0 & 0 & 0 & 0 & 0 & 0 & 0 \\
0 & 0 & 0 & 0 & 0 & 0 & 0 \\
0 & 0 & 0 & 0 & 0 & 0 & 0 \\
0 & 0 & 0 & 0 & 0 & 0 & 0 \\
0 & 0 & 0 & 0 & 0 & 0 & 0 \\
-\frac{1}{2} & 0 & 0 & 0 & 0 & 0 & 0
\end{array}\right) \\
& \Phi_{9}=\left(\begin{array}{ccccccc}
0 & 0 & 0 & 0 & 0 & 0 & 0 \\
0 & 0 & 0 & 0 & 0 & 0 & \frac{\gamma_{3}}{2 \gamma_{1}} \\
0 & 0 & 0 & 0 & 0 & 0 & 0 \\
0 & 0 & 0 & 0 & 0 & 0 & 0 \\
0 & 0 & 0 & 0 & 0 & 0 & 0 \\
0 & 0 & 0 & 0 & 0 & 0 & 0 \\
0 & -\frac{1}{2} & 0 & 0 & 0 & 0 & 0
\end{array}\right) \quad \Phi_{10}=\left(\begin{array}{ccccccc}
0 & 0 & 0 & 0 & 0 & 0 & 0 \\
0 & 0 & 0 & 0 & 0 & 0 & 0 \\
0 & 0 & 0 & 0 & 0 & 0 & \frac{\gamma_{3}}{2 \gamma_{1}} \\
0 & 0 & 0 & 0 & 0 & 0 & 0 \\
0 & 0 & 0 & 0 & 0 & 0 & 0 \\
0 & 0 & 0 & 0 & 0 & 0 & 0 \\
0 & 0 & -\frac{1}{2} & 0 & 0 & 0 & 0
\end{array}\right)
\end{aligned}
$$

and $\Phi_{11}=0$. 
Let $S=K_{3}+\alpha K_{11}$, where $\alpha \in \mathbb{R}$ is a parameter. Then $S$ is given by the data $(\xi, \Phi)$ with

$$
\xi=\alpha e_{7} \quad \text { and } \quad \Phi=\Phi_{3} .
$$

We claim that for some choice of metric, $S$ is the Reeb vector field of a Sasakian structure on $P$. We recall that an odd-dimensional riemannian manifold $(P, g)$ is Sasakian if and only if its metric cone $\left(\widetilde{P}=\mathbb{R}^{+} \times P, \widetilde{g}=d r^{2}+r^{2} g\right)$, for $r>0$ the coordinate on $\mathbb{R}^{+}$, is Kähler. Let us see what this means intrinsically.

On $\widetilde{P}$ there is a hermitian structure $J$ which is compatible with $\widetilde{g}$ and is parallel relative to the riemannian connection $\widetilde{\nabla}$ of $\widetilde{g}$. Let $\omega$ denote the associated Kähler form: $\omega(X, Y)=\widetilde{g}(J X, Y)$. Let $E=r \frac{\partial}{\partial r}$ denote the Euler vector field. Its derivative $\widetilde{\nabla} E$ is the identity endomorphism: $\widetilde{\nabla}_{X} E=X$ for all vector fields $X$ on the cone, whence it generates homotheties of $(\widetilde{P}, \widetilde{g})$. Define a 1-form $\widetilde{\eta}$ on the cone by $\widetilde{\eta}=\iota_{E} \omega$. In other words, $\widetilde{\eta}(X)=\omega(E, X)=\widetilde{g}(J E, X)$. A quick calculation shows that $\widetilde{\eta}$ scales with weight 2 under the homotheties generated by $E$. Since $\iota_{E} \widetilde{\eta}=0$, we see that $r^{-2} \widetilde{\eta}$ is basic; that is, there is a 1-form $\eta$ on $P$ such that $r^{-2} \widetilde{\eta}=\pi^{*} \eta$ with $\pi: \widetilde{P} \rightarrow P$ the natural projection $(r, p) \mapsto p$. It is another relatively straightforward calculation to show that $d \widetilde{\eta}=2 \omega$, whence

$$
\omega=\frac{1}{2} d \widetilde{\eta}=\frac{1}{2} d\left(r^{2} \pi^{*} \eta\right)=r d r \wedge \pi^{*} \eta+\frac{1}{2} r^{2} \pi^{*} d \eta .
$$

Let $P$ be $(2 n+1)$-dimensional. Since $\omega$ is a Kähler form,

$$
\omega^{n+1}=n 2^{-n} r^{2 n+1} \pi^{*}\left(\eta \wedge(d \eta)^{n}\right)
$$

is nowhere vanishing, which implies that $\eta \wedge(d \eta)^{n}$ is nowhere vanishing and thus $\eta$ defines a contact structure on $P$.

Let us define the vector field $\widetilde{S}=J E$. Since $\widetilde{g}(\widetilde{S}, E)=\widetilde{g}(J E, E)=0, \widetilde{S}$ restricts to a vector field on $P$, which we may and will think of as the $r=1$ slice of $\widetilde{P}$. It is an easy calculation to show that $\widetilde{S}$ is a Killing vector field with norm $r^{2}$, whence it restricts to a unit-norm Killing vector $S$ on $P$ and hence $\eta(S)=1$. The covariant derivative $\phi=-\nabla S$ of $S$ on $M$ defines a complex structure on the distribution $\mathscr{D}$ orthogonal to the one spanned by $S$ itself. Indeed, $\widetilde{\nabla} \widetilde{S}=\widetilde{\nabla} J E=J \circ \widetilde{\nabla} E=J$ and hence $\phi$ is defined by declaring it to coincide with $J$ on the orthogonal complement to the distribution spanned by $E$ and $\widetilde{S}$ and to annihilate $S: \phi(S)=0$. In other words, one can show that

$$
\phi^{2}=-\mathrm{id}+S \otimes \eta .
$$

The compatibility between the riemannian and complex structures on the cone becomes the compatibility between the riemannian and contact structures on $P$; namely,

$$
g(\phi X, \phi Y)=g(X, Y)-\eta(X) \eta(Y) .
$$

This simply says that $\phi$ is an isometry on the distribution $\mathscr{D}$.

Finally, the integrability condition of the hermitian structure (i.e., the vanishing of the Nijenhuis tensor) becomes a differential condition on $\phi$ :

$$
\left(\nabla_{X} \phi\right)(Y)=g(X, Y) S-g(S, Y) X .
$$


We may rewrite the left-hand side as

$$
\left(\nabla_{X} \phi\right)(Y)=\nabla_{X}(\phi(Y))-\phi\left(\nabla_{X} Y\right)=-\nabla_{X} \nabla_{Y} S+\nabla_{\nabla_{X} Y} S=-R(S, X) Y,
$$

whence the integrability condition becomes an algebraic equation involving the curvature tensor:

$$
R(S, X) Y+g(X, Y) S-g(S, Y) X=0
$$

A Sasaki structure is actually too strict for our needs. This derives from the fact that Sasaki structures are not scale invariant, whereas the supergravity field equations are. We actually need a somewhat more relaxed notion of Sasaki structure which says that $(P, g, \eta, S, \phi)$ is only homothetic to a Sasaki structure. In other words, we (tentatively) say that $(P, g, \eta, S, \phi)$ defines an $r$-Sasaki structure, if for some $r>0,\left(P, r^{2} g, \eta, S, \phi\right)$ is a Sasaki structure. The name comes from the fact that the $r$-slice of a Kähler cone has such a structure. Since both the riemannian connection $\nabla$ and the riemann curvature $R$ are invariant under homotheties, $(P, g, \eta, S, \phi)$ is an $r$-Sasaki structure if all equations of a Sasaki structure are obeyed, except for the following changes:

1. the normalisation of $S$ is now $g(S, S)=r^{-2}$,

2. the metric compatibility condition (8.7) is now

$$
g(\phi X, \phi Y)=g(X, Y)-r^{-2} \eta(X) \eta(Y),
$$

3. and the integrability condition (8.12) is replaced by

$$
R(S, X) Y+r^{2} g(X, Y) S-r^{2} g(S, Y) X=0 .
$$

These conditions are easy to check for the homogeneous backgrounds of interest. We notice that the new integrability condition (8.12) is tensorial and only depends on the value of the Reeb vector field $S$ at the origin and does so linearly. Therefore in the expression (8.3) for $S$, the parameter $\alpha$ is not fixed by (8.12). Indeed, it is not difficult to verify that for all $\alpha \neq 0$, the integrability condition (8.12) is satisfied provided that $r^{2}=\frac{\gamma_{3}}{4 \gamma_{1}^{2}}$. The parameter $\alpha$ is fixed by normalising $S$ to $g(S, S)=r^{-2}$, which means $\alpha=\frac{2 \gamma_{1}}{\gamma_{3}}$. Comparing with (8.1) we see that $S=\chi$ the generator of the centre of the isometry Lie algebra.

In the case of the background with flux given by equation (7.29), the Reeb vector field $S$ does not preserve it, whence the symmetry Lie algebra of the background is precisely $\mathfrak{s o}(5) \oplus$ $\mathfrak{s o}(3,2)$. Let us briefly explain the calculation of the Lie derivative $L_{S} F$ of $F$ along $S$. Using the Cartan formula and the fact that $F$ is closed, $L_{S} F=d i_{S} F$, so we need to compute the exterior derivative of the 3 -form $i_{S} F$. We saw in section 2.2, particularly equation (2.15), that the exterior derivative of an invariant form is easy to compute algebraically. Now let $X$ be a Killing vector and let us see whether $i_{S} F$ is invariant. We calculate the Lie derivative of $i_{S} F$ along $X$ to obtain

$$
L_{X} i_{S} F=i_{S} L_{X} F+i_{[X, S]} F
$$


The first term in the r.h.s. vanishes because $F$ is invariant and the second term vanishes precisely because the Reeb vector field $S$ is central, whence $[X, S]=0$ for all Killing vectors $X$. This means that we can use equation (2.15) to compute $d i_{S} F$ and we find that it is not zero.

\subsection{Other $n=5$ backgrounds}

We now look in some detail at two of the backgrounds found in section 6.3.

\subsubsection{A supersymmetric Freund-Rubin background}

In this background, the geometry is $S^{4} \times X^{7}$ with $X$ a lorentzian Sasaki-Einstein manifold. Since the background is Freund-Rubin, this means [34] that it is supersymmetric. Explicitly, in terms of the basis given earlier in this section, and reintroducing the scale $\lambda \neq 0$, we have

$$
\begin{aligned}
\lambda^{-2} g & =-\left(\theta^{0}\right)^{2}+\frac{4}{9}\left(\left(\theta^{1}\right)^{2}+\cdots+\left(\theta^{4}\right)^{2}\right)+\frac{2}{3}\left(\left(\theta^{5}\right)^{2}+\cdots+\left(\theta^{\natural}\right)^{2}\right) \\
\lambda^{-3} F & =\frac{8}{9} \theta^{1234} .
\end{aligned}
$$

Let us now show that $X$ admits an invariant lorentzian Sasaki-Einstein structure. In fact, let us consider more generally any homogeneous lorentzian geometry of the type $(\mathfrak{s o}(3,2), \mathfrak{s o}(3))$ with $\gamma_{2}=\gamma_{3}=: \gamma$. This describes two of the three backgrounds we have found. Lorentzian Sasaki structures $(M, g, S, \eta, \phi)$ are described by a lorentzian odddimensional manifold $(M, g)$ with a timelike Killing vector $S$ normalised to $g(S, S)=-1$, a contact structure $\eta$ with $\eta(S)=1$ and endomorphism $\phi=-\nabla S$ with $\phi^{2}=-\mathrm{id}+S \otimes \eta$ and subject to slight modifications of the metric compatibility and integrability conditions (8.7) and (8.12), respectively; namely, the lorentzian metric compatibility condition is now

$$
g(\phi X, \phi Y)=g(X, Y)+\eta(X) \eta(Y)
$$

whereas the integrability condition reads

$$
R(S, X) Y-g(X, Y) S+g(S, Y) X=0 .
$$

Similarly, we can consider lorentzian $r$-Sasaki structures $(M, g, S, \eta, \phi)$, defined in such a way that $\left(M, r^{2} g, S, \eta, \phi\right)$ is lorentzian Sasaki. This means that now $S$ is normalised to $g(S, S)=-r^{-2}$ and that the metric compatibility and integrability conditions change to

$$
g(\phi X, \phi Y)=g(X, Y)+r^{-2} \eta(X) \eta(Y)
$$

whereas the integrability condition reads

$$
R(S, X) Y-r^{2} g(X, Y) S+r^{2} g(S, Y) X=0 .
$$

The situation here is very similar to that of section 8.1. When $\gamma_{2}=\gamma_{3}$, there is an enhancement of symmetry to $\mathfrak{s o}(3,2) \oplus \mathfrak{s o}(2)$, where the central Killing vector $S$ is 
determined by the pair $(\xi, \Phi) \in \mathfrak{m} \oplus \mathfrak{s o}(\mathfrak{m})$, with $\xi=2 \gamma J_{45}$ and $\Phi$ given relative to the ordered basis $\left(J_{i 4}, J_{i 5}, J_{45}\right)$ by the matrix

$$
\left(\begin{array}{ccccccc}
0 & 0 & 0 & 1 & 0 & 0 & 0 \\
0 & 0 & 0 & 0 & 1 & 0 & 0 \\
0 & 0 & 0 & 0 & 0 & 1 & 0 \\
-1 & 0 & 0 & 0 & 0 & 0 & 0 \\
0 & -1 & 0 & 0 & 0 & 0 & 0 \\
0 & 0 & -1 & 0 & 0 & 0 & 0 \\
0 & 0 & 0 & 0 & 0 & 0 & 0
\end{array}\right) .
$$

We claim, as suggested by the choice of notation, that $S$ is the Reeb vector field of a lorentzian $r$-Sasaki structure. It is normalised to $g(S, S)=-4 \gamma^{2}$, whence we expect that $r=\frac{1}{2 \gamma}$ and indeed a straightforward calculation shows that

$$
4 \gamma^{2} R(S, X) Y-g(X, Y) S+g(S, Y) X=0 .
$$

The nonzero components of the Levi-Civita connection for this metric are readily calculated using the formulas (2.8) and (6.7):

$$
\begin{array}{ll}
\nabla_{J_{45}} J_{i 4}=\left(\frac{1}{2 \gamma}-1\right) J_{i 5} & \nabla_{J_{45}} J_{i 5}=\left(1-\frac{1}{2 \gamma}\right) J_{i 4} \\
\nabla_{J_{i 4}} J_{45}=\frac{1}{2 \gamma} J_{i 5} & \nabla_{J_{i 5}} J_{45}=-\frac{1}{2 \gamma} J_{i 4} \\
\nabla_{J_{i 4}} J_{j 5}=\frac{1}{2} \delta_{i j} J_{45} & \nabla_{J_{i 5}} J_{j 4}=-\frac{1}{2} \delta_{i j} J_{45} .
\end{array}
$$

Using the formulae (6.8) in the case where $\gamma_{2}=\gamma_{3}=: \gamma$, we see that $\Lambda=1-4 \gamma^{2}$ and

$$
\operatorname{Ric}\left(J_{45}, J_{45}\right)=\frac{3}{2 \gamma^{2}} \quad \text { and } \quad \operatorname{Ric}\left(J_{i 4}, J_{j 4}\right)=\operatorname{Ric}\left(J_{i 5}, J_{j 5}\right)=\delta_{i j}\left(\frac{1}{2 \gamma}-3\right),
$$

whence the metric is Einstein if and only if $-\frac{3}{2 \gamma^{2}}=\frac{1}{\gamma}\left(\frac{1}{2 \gamma}-3\right)$, or equivalently if and only if $\gamma=\frac{2}{3}$.

In summary, we have shown that the background with $\gamma_{1}=\frac{4}{9}$ and $\gamma_{2}=\gamma_{3}=\frac{2}{3}$ is a Freund-Rubin background of the form $S^{4} \times X^{7}$, with $X$ (homothetic to) a lorentzian Sasaki-Einstein manifold. Lischewski [35] has shown that this background admits $N=2$ supersymmetry, a fact that can also be deduced in this case from unpublished results [34]. Indeed, Killing spinors take the form

$$
\varepsilon^{I a}=\zeta^{I a} \otimes \psi,
$$

where $I=1, \ldots, 4$ and $a=1,2, \zeta^{I a}$ are geometric Killing spinors on $S^{4}$ and $\psi$ is a geometric Killing spinor on the lorentzian Sasaki-Einstein manifold. The spinor $\varepsilon$ is subject to a symplectic Majorana condition

$$
\left(\varepsilon^{I a}\right)^{*}=\Omega_{I J} \epsilon_{a b} \varepsilon^{J b},
$$

with $\Omega_{I J}$ the $\operatorname{Sp}(2)$-invariant symplectic structure on the space of Killing spinors of $S^{4}$, which by Bär's cone construction is isomorphic as an $\mathrm{Sp}(2)$-module to the space of parallel 
spinors on $\mathbb{R}^{5}$, which is just the spinor irreducible representation of $\operatorname{Spin}(5) \cong \operatorname{Sp}(2)$, hence a quaternionic representation. With a suitable normalisation of the Killing spinors, the Killing superalgebra is given by

$$
\left[\varepsilon^{I a}, \varepsilon^{J b}\right]=\Omega^{I J} \epsilon^{a b} \chi
$$

where $\chi$ is the Reeb vector field of the lorentzian Sasaki structure. Therefore the $\mathrm{SO}(3,2) \times$ $\mathrm{SO}(5)$ symmetry is accidental and only the central $\mathrm{SO}(2)$ symmetry is induced by the supersymmetry.

This solution looks like it could be obtained via a Wick rotation from a background of the type $\mathrm{AdS}_{4} \times X^{7}$, with $X$ a Sasaki-Einstein 7 -manifold. ${ }^{2}$ If that is the case, the $\mathrm{AdS}_{4}$ background must be a Freund-Rubin background and hence must be one of the backgrounds classified in [28] and discussed here in section 7.4.2. Let us try to identify it. The solution we found is described, as a homogeneous space, by the data $(\mathfrak{g}, \mathfrak{h})=$ $(\mathfrak{s o}(5) \oplus \mathfrak{s o}(3,2), \mathfrak{s o}(4) \oplus \mathfrak{s o}(3))$. We may think of $\mathfrak{s o}(5) \oplus \mathfrak{s o}(3,2)$ as a real Lie subalgebra of the complex Lie algebra $\mathfrak{g l}(10, \mathbb{C})$ of $10 \times 10$ complex matrices. We are after a homogeneous $\mathrm{AdS}_{4}$ Freund-Rubin background which, as a homogeneous space is described algebraically by the data $\left(\mathfrak{g}^{\prime}, \mathfrak{h}^{\prime}\right)=(\mathfrak{s o}(3,2) \oplus \mathfrak{s o}(5), \mathfrak{s o}(3,1) \oplus \mathfrak{s o}(3))$. Therefore the Wick rotation we are after is an element $\varpi \in \mathrm{GL}(10, \mathbb{C})$ such that multiplying on both the left and the right by $\varpi$ maps $(\mathfrak{g}, \mathfrak{h})$ to $\left(\mathfrak{g}^{\prime}, \mathfrak{h}^{\prime}\right)$. A little experimentation leads us to the following diagonal matrix

$$
\varpi=\left(\begin{array}{llll}
I_{3} & & & \\
& i I_{2} & & \\
& & I_{3} & \\
& & & i I_{2}
\end{array}\right) \in \operatorname{GL}(10, \mathbb{C}),
$$

where $I_{n}$ is the $n \times n$ identity matrix. The element $\varpi$ thus defines a "quadruple" Wick rotation. The map $X \mapsto \varpi X \varpi$ sends the Lie subalgebra $\mathfrak{g}=\mathfrak{s o}(5) \oplus \mathfrak{s o}(3,2) \subset \mathfrak{g l}(10, \mathbb{C})$ which preserves the inner product

$$
\eta=\left(\begin{array}{llll}
I_{3} & & & \\
& I_{2} & & \\
& & I_{3} & \\
& & & -I_{2}
\end{array}\right)
$$

to the isomorphic Lie subalgebra $\mathfrak{g}^{\prime}=\mathfrak{s o}(3,2) \oplus \mathfrak{s o}(5)$ which preserves the inner product

$$
\eta^{\prime}=\left(\begin{array}{llll}
I_{3} & & & \\
& -I_{2} & & \\
& & I_{3} & \\
& & & I_{2}
\end{array}\right) .
$$

At the same it sends the subalgebra $\mathfrak{h}=\mathfrak{s o}(4) \oplus \mathfrak{s o}(3)$ of $\mathfrak{g}$ to the subalgebra $\mathfrak{h}^{\prime}=\mathfrak{s o}(3,1) \oplus$ $\mathfrak{s o}(3)$ of $\mathfrak{g}^{\prime}$. It is not hard to show that the homogeneous space described by $\left(\mathfrak{g}^{\prime}, \mathfrak{h}^{\prime}\right)$ admits a

\footnotetext{
${ }^{2}$ We are grateful to James Lucietti for this suggestion.
} 
Freund-Rubin background, given relative to the Wick-rotated basis by a similar expression to that of (8.14), namely

$$
\begin{aligned}
\lambda^{-2} g & =\left(\theta^{0}\right)^{2}+\frac{4}{9}\left(\left(\theta^{1}\right)^{2}+\left(\theta^{2}\right)^{2}+\left(\theta^{3}\right)^{2}-\left(\theta^{4}\right)^{2}\right)+\frac{2}{3}\left(\left(\theta^{5}\right)^{2}+\cdots+\left(\theta^{\natural}\right)^{2}\right) \\
\lambda^{-3} F & =\frac{8}{9} \theta^{1234} .
\end{aligned}
$$

The underlying geometry is $\mathrm{AdS}_{4} \times X^{7}$, where $X^{7}$ is a Sasaki-Einstein manifold with isometry Lie algebra $\mathfrak{s o}(5) \oplus \mathfrak{s o}(2)$ because of the enhancement due to $\gamma_{2}=\gamma_{3}$. In fact, it is possible to identify $X^{7}$ with the real Stiefel manifold $V_{2}\left(\mathbb{R}^{5}\right)$ of orthonormal 2-frames in $\mathbb{R}^{5}$ with the Einstein metric, equivalently the unit tangent bundle to $S^{4}$. This background is discussed in ([28], appendix C) and is also discussed in [36], which contains references to earlier papers. It is shown in [28] that the solution has $N=2$ supersymmetry, just as the Wick-rotated background found here.

\subsubsection{A circle of backgrounds}

This background depends on a parameter $\alpha$ which shares the same underlying geometry:

$$
\begin{aligned}
\lambda^{-2} g= & -\left(\theta^{0}\right)^{2}+\frac{4}{9}\left(\left(\theta^{1}\right)^{2}+\cdots+\left(\theta^{\natural}\right)^{2}\right) \\
\lambda^{-3} F= & -\frac{1}{3} \theta^{1234}+\frac{1}{\sqrt{3}} \cos \alpha\left(\theta^{0567}-\theta^{059 \natural}+\theta^{068 \natural}-\theta^{0789}\right) \\
& -\frac{1}{\sqrt{3}} \sin \alpha\left(\theta^{056 \natural}-\theta^{0579}+\theta^{0678}-\theta^{089 \natural}\right) \\
= & -\frac{1}{3} \theta^{1234}+\frac{1}{\sqrt{3}} \theta^{0} \wedge \operatorname{Re}\left(e^{i \alpha}\left(\theta^{5}+i \theta^{8}\right) \wedge\left(\theta^{6}+i \theta^{9}\right) \wedge\left(\theta^{7}+i \theta^{\natural}\right)\right) .
\end{aligned}
$$

The geometry is again $S^{4} \times X^{7}$ with $X$ (homothetic to) a lorentzian Sasaki manifold, but now it is not Einstein. This does not necessarily imply that it is not supersymmetric, since the background is not of Freund-Rubin type: the 4-form has components in both factors. However Lischewski [35] has shown that this background is not supersymmetric by an explicit calculation of the holonomy algebra of the connection $D$. This background does not seem to be Wick-related to an $A d S_{4}$ background. In the second expression for $F$ we recognise a transverse special lagrangian calibration, which suggests that this background is obtained from a supersymmetric Freund-Rubin background via the Englert procedure. It seems likely that the supersymmetric Freund-Rubin background in question is the background described in the previous section. Finally, we remark that although there is an enhancement of the isometry algebra by an additional central Killing vector, this is not a symmetry of $F$, whence the symmetry Lie algebra of the background remains isomorphic to $\mathfrak{s o}(3,2) \oplus \mathfrak{s o}(5)$.

\section{Summary of results and open problems}

We have presented the results of a systematic search for eleven-dimensional supergravity backgrounds homogeneous under a Lie group with Lie algebra $\mathfrak{g}_{n}:=\mathfrak{s o}(n) \oplus \mathfrak{s o}(3,2)$ for $n=5,6,7$. The aim of this search is to explore the existence of new candidate backgrounds with $N>4$ supersymmetry dual to three-dimensional superconformal field theories. It is 
known that such backgrounds are homogeneous and the structure of the superconformal algebra is such that the bosonic subalgebra is isomorphic to $\mathfrak{g}_{n}$. Since backgrounds with $N=8$ supersymmetry have been classified, we have restricted ourselves to $n=5,6,7$; although we have many partial results for $n=4$ which have not made it to this paper.

Such homogeneous backgrounds come in two families: those with underlying geometry $\mathrm{AdS}_{4} \times P^{7}$ and the rest. We find no new backgrounds for $n=6,7$, but we find a number of possibly novel backgrounds with $n=5$ of both types. Curiously all backgrounds we find are metrically products.

We find three new backgrounds with underlying geometry $\mathrm{AdS}_{4} \times P^{7}$, where $P$ is a homogeneous riemannian manifold $\mathrm{SO}(5) / \mathrm{SO}(3)$, where $\mathrm{SO}(3)$ is the subgroup of $\mathrm{SO}(5)$ which leaves pointwise invariant a plane in $\mathbb{R}^{5}$. One of the backgrounds can only be approximated numerically. Of the other two backgrounds, one of them is discussed in detail in section 8.1, where it is shown that it is (homothetic to) a Sasaki manifold, whence the geometry has an enhanced isometry Lie algebra $\mathfrak{s o}(3,2) \oplus \mathfrak{s o}(5) \oplus \mathfrak{s o}(2)$, where $\mathfrak{s o}(2)$ is generated by the Reeb vector field of the Sasaki structure. The flux is not preserved by the Reeb vector field, whence the background's symmetry is not enhanced. In fact, this background is not new, since its existence was mentioned in ([28], appendix C). It can be identified with the result of applying the Englert procedure to a background $\operatorname{AdS}_{4} \times V_{2}\left(\mathbb{R}^{5}\right)$. As a result it breaks all the supersymmetry. We have not analysed the supersymmetry of the other two backgrounds. Moreover, there is a small gap in these results, in that we have not been able to complete the search for backgrounds with underlying geometry $\mathrm{AdS}_{4} \times P^{7}$, where $P$ is a homogeneous riemannian manifold $\mathrm{SO}(5) / \mathrm{SO}(3)_{\text {irr }}$, where $\mathrm{SO}(3)_{\text {irr }}$ is a maximal subgroup acting irreducibly on $\mathbb{R}^{5}$.

We also have found backgrounds which do not have an $\mathrm{AdS}_{4}$ factor, yet still have an $\mathfrak{s o}(3,2)$ summand in the symmetry algebra. We have found three such backgrounds, all with underlying geometry $S^{4} \times Q^{7}$ with $S^{4}$ the round 4-sphere and $Q$ a homogeneous lorentzian manifold $\mathrm{SO}(3,2) / \mathrm{SO}(3)$ but with different kinds of fluxes. One of the backgrounds, discussed in detail in section 8.2.1 is of Freund-Rubin type since the flux is proportional to the volume form on $S^{4}$. In this case $Q$ is (homothetic to) a lorentzian Sasaki-Einstein manifold and this means that the background is supersymmetric, albeit only with $N=2$. As shown in section 8.2.1, this background is Wick-related to a Freund-Rubin background $\mathrm{AdS}_{4} \times V_{2}\left(\mathbb{R}^{5}\right)$ already known from classical times [28]. There is an enhancement of symmetry and the full isometry algebra is $\mathfrak{s o}(5) \oplus \mathfrak{s o}(3,2) \oplus \mathfrak{s o}(2)$, with the $\mathfrak{s o}(2)$ generated by the Reeb vector field of the Sasaki structure. We also find find a circle's worth of backgrounds, described in section 8.2.2, which seems to be the result of applying the Englert procedure to the Freund-Rubin background just mentioned. If this is indeed the case, then the background preserves no supersymmetry. Here the geometry is lorentzian Sasaki and although there is an enhancement of the isometry algebra to $\mathfrak{s o}(5) \oplus \mathfrak{s o}(3,2) \oplus \mathfrak{s o}(2)$, the Reeb vector field does not preserve the rather complicated flux. Finally, we also find a background which we can only approximate numerically. For this background there is no enhancement of the symmetry and in particular $Q$ does not have a homogeneous Sasaki structure. This numerical background is given by equation (6.25) and we have yet to investigate whether it preserves any supersymmetry. 


\section{Acknowledgments}

The work of JMF was supported in part by grants ST/G000514/1 "String Theory Scotland" and ST/J000329/1 "Particle Theory at the Tait Institute" from the U.K. Science and Technology Facilities Council and that of MU in part by the Nuffield Undergraduate Research Bursary URB/39258. We are grateful to both institutions for their support. Twice during the long gestation of this project, JMF visited the University of Tokyo and he would like to thank Teruhiko Kawano for organising the visits and for providing such a pleasant research environment. We are also grateful to Christoph Nölle for some initial collaboration on this project, as well as to Paul de Medeiros, Joan Simón and especially James Lucietti for lending us their ears. We are particularly grateful to Robert Bryant for his answer to a question on MathOverflow, recapped in the appendix, and on which some of the calculations in section 8 are based; and to Andree Lischewski for developing an algorithm to check the supersymmetry of a homogeneous background and for performing some checks on the results of section 8. Finally, we are grateful to Dima Sorokin for making us aware of his early work on supergravity compactifications.

\section{A Isometries of a homogeneous space}

Let $(M, g)$ be a homogeneous riemannian manifold admitting a transitive action of $G$ with generic stabiliser $H$, so that $M$ is diffeomorphic to $G / H$. This means that $G$ is a subgroup of the isometry group of $(M, g)$, but it could very well be the case that $G$ is a proper subgroup. The Lie algebra of the group of isometries can be determined by solving the Killing vector equation on $(M, g)$. A Killing vector is determined uniquely by its value at a point and that of its covariant derivative relative to the Levi-Civita connection. Indeed, as shown in [37, 38] and discussed in [39], Killing vectors are in bijective correspondence with parallel sections of $T M \oplus \mathfrak{s o}(T M)$, with $\mathfrak{s o}(T M) \cong \Lambda^{2} T^{*} M$ the bundle of skewsymmetric endomorphisms of the tangent bundle, relative to the connection defining the so-called Killing transport:

$$
D_{X}\left(\begin{array}{c}
\xi \\
A
\end{array}\right)=\left(\begin{array}{c}
\nabla_{X} \xi+A(X) \\
\nabla_{X} A-R(X, \xi)
\end{array}\right) .
$$

In a homogeneous space, since both $\nabla$ and $R$ are invariant under isometries, it is possible to turn this into a linear system of equations with constant coefficients, which can be succinctly described by lifting the problem to the group $G$. The following treatment owes a lot to Robert Bryant [40] via MathOverflow.

As usual we think of $(M, g)$ as described algebraically by a reductive split $\mathfrak{g}=\mathfrak{h} \oplus \mathfrak{m}$ together with an $H$-invariant inner product $\langle-,-\rangle$ on $\mathfrak{m}$. Let us choose bases $\left(X_{a}\right)$ for $\mathfrak{h}$ and $\left(Y_{i}\right)$ for $\mathfrak{m}$. The structure constants of $\mathfrak{g}$ relative to these bases are given by

$$
\left[X_{a}, X_{b}\right]=f_{a b}{ }^{c} X_{c} \quad\left[X_{a}, Y_{i}\right]=f_{a i}{ }^{j} Y_{j} \quad\left[Y_{i}, Y_{j}\right]=f_{i j}{ }^{a} X_{a}+f_{i j}{ }^{k} Y_{k} .
$$

Let $\left(\psi^{a}\right)$ and $\left(\theta^{i}\right)$ denote the left-invariant Maurer-Cartan one-forms on $G$ dual to the chosen bases for $\mathfrak{g}$. The structure equations are

$$
d \psi^{c}=-\frac{1}{2} f_{a b}^{c} \psi^{a} \wedge \psi^{b}-\frac{1}{2} f_{i j}{ }^{c} \theta^{i} \wedge \theta^{j} \quad d \theta^{k}=-\frac{1}{2} f_{i j}{ }^{k} \theta^{i} \wedge \theta^{j}-f_{a i}{ }^{k} \psi^{a} \wedge \theta^{i} .
$$


The $H$-invariant inner product on $\mathfrak{m}$ has components $\eta_{i j}:=\left\langle Y_{i}, Y_{j}\right\rangle$ relative to the chosen basis and $\eta_{i j} \theta^{i} \theta^{j}$ is the pullback to $G$ of the invariant metric on $M=G / H$. The invariance of the inner product means that $f_{a i j}=-f_{a j i}$, where here and in the sequel, we lower indices using $\eta_{i j}$, so that $f_{a i j}=f_{a i}{ }^{k} \eta_{k j}$.

Let $\omega^{i}{ }_{j}$ denote the connection 1-form defined by

$$
d \theta^{i}=-\omega^{i}{ }_{j} \wedge \theta^{j} \quad \text { and } \quad \omega_{i j}=-\omega_{j i} .
$$

The structure equations allow us to solve for $\omega^{i}{ }_{j}$ :

$$
\omega^{i}{ }_{j}=f_{a j}{ }^{i} \psi^{a}-\frac{1}{2} f_{j k}{ }^{i} \theta^{k}+\frac{1}{2} \eta^{i l}\left(f_{l j k}+f_{l k j}\right) \theta^{k} .
$$

The curvature 2-form

$$
\Omega^{i}{ }_{j}=d \omega^{i}{ }_{j}+\omega^{i}{ }_{k} \wedge \omega^{k}{ }_{j}
$$

can be shown to be horizontal, whence it can be expressed only in terms of the $\left(\theta^{i}\right)$ :

$$
\Omega^{i}{ }_{j}=\frac{1}{2} R_{j k l}^{i} \theta^{k} \wedge \theta^{l} .
$$

A Killing vector field on $M=G / H$ lifts to a vector field on $G$ which is defined by the data $(\xi, A)$, where

$$
d \xi^{i}+\omega^{i}{ }_{j} \xi^{j}=-A^{i}{ }_{j} \theta^{j} \quad \text { and } \quad A_{i j}=-A_{j i} .
$$

Differentiating this equation and using the structure equations (A.4), (A.6) and Killing's equation (A.8) itself, we arrive at

$$
\left(d A^{i}{ }_{j}+[\omega, A]_{j}^{i}\right) \wedge \theta^{j}=-\Omega^{i}{ }_{j} \xi^{j} .
$$

The following simple result is very useful.

Lemma. Let $M^{i}{ }_{j}$ be a matrix of 1-forms such that

$$
M^{i}{ }_{j} \wedge \theta^{j}=0 \quad \text { and } \quad M_{i j}=-M_{j i} .
$$

Then $M=0$.

Proof. Write $M^{i}{ }_{j}=M^{i}{ }_{j k} \theta^{k}$. The condition $M^{i}{ }_{j} \wedge \theta^{j}=0$ becomes $M^{i}{ }_{j k} \theta^{k} \wedge \theta^{j}=0$, which is equivalent to $M^{i}{ }_{j k}=M^{i}{ }_{k j}$. Lowering the index with $\eta$, this is equivalent to $M_{i j k}=M_{i k j}$; but since $M_{i j k}=-M_{j i k}$, we see that

$$
M_{i j k}=-M_{j i k}=-M_{j k i}=M_{k j i}=M_{k i j}=-M_{i k j}=-M_{i j k} \quad \text { hence } \quad M_{i j k}=0 .
$$

Equation (A.9) says that $\Omega^{i}{ }_{j} \xi^{j}+\rho^{i}{ }_{j} \wedge \theta^{j}=0$, where $\rho^{i}{ }_{j}=d A^{i}{ }_{j}+[\omega, A]^{i}{ }_{j}$. Using the lemma, we can give an alternate expression for $\rho$ in terms of the curvature. Indeed,

$$
\begin{aligned}
\Omega^{i}{ }_{j} \xi^{j}+\rho^{i}{ }_{l} \wedge \theta^{l} & =\frac{1}{2} R^{i}{ }_{j k l} \xi^{j} \theta^{k} \wedge \theta^{l}+\rho_{l}^{i} \wedge \theta^{l} \\
& =\left(\frac{1}{2} R^{i}{ }_{j k l} \xi^{j} \theta^{k}+\rho^{i}{ }_{l}\right) \wedge \theta^{l}
\end{aligned}
$$


but the algebraic Bianchi identity says that $R_{j k l}^{i}=-R_{l j k}^{i}-R_{k l j}^{i}$, whence

$$
\begin{aligned}
\frac{1}{2} R_{j k l}^{i} \xi^{j} \theta^{k} \wedge \theta^{l} & =-\frac{1}{2}\left(R_{l j k}^{i}+R_{k l j}^{i}\right) \xi^{j} \theta^{k} \wedge \theta^{l} \\
& =R_{l k j}^{i} \xi^{j} \theta^{k} \wedge \theta^{l} .
\end{aligned}
$$

This implies that

$$
\left(R_{l k j}^{i} \xi^{j} \theta^{k}+\rho_{l}^{i}\right) \wedge \theta^{l}=0,
$$

but since $R_{i j k l}=-R_{j i k l}$ and $\rho_{i j}=-\rho_{j i}$, the lemma says that

$$
\rho_{l}^{i}=R_{l j k}^{i} \xi^{j} \theta^{k} .
$$

It is convenient to think of $\rho$ as a bilinear in $\xi$ and $\theta$ and define

$$
\rho(\xi, \theta)^{i}{ }_{l}=R^{i}{ }_{l j k} \xi^{j} \theta^{k} .
$$

Therefore equation (A.9) together with the lemma, imply that

$$
d A+[\omega, A]=\rho(\xi, \theta),
$$

where we have dropped the indices and interpreted this equation as a matricial equation.

Differentiating equation (A.15) and using the various structure equations to eliminate the derivatives $d \xi, d A, d \omega$ and $d \theta$ from the expression, we arrive at

$$
[\Omega, A]-[\omega, \rho(\xi, \theta)]+\rho(\xi, \omega \wedge \theta)+\rho(\omega \xi, \theta)+\rho(A \theta, \theta)=0,
$$

where $[\Omega, A]=\Omega A-A \Omega$ and $[\omega, \rho(\xi, \theta)]=\omega \wedge \rho(\xi, \theta)+\rho(\xi, \theta) \wedge \omega$. The beauty of equation (A.16) is that it is linear on $\xi, A$ with constant coefficients!

Differentiating further and using the various structure equations again to eliminate derivatives, yields new linear equations with constant coefficients. Eventually this process will terminate, in the sense that no new equations are obtained. When this happens, we are left with a set of linear equations in $(\xi, A)$ whose solution space is the Lie algebra of isometries of $(M, g)$ with Lie bracket given by

$$
\left[\left(\xi_{1}, A_{1}\right),\left(\xi_{2}, A_{2}\right)\right]=\left(A_{1} \xi_{2}-A_{2} \xi_{1},\left[A_{1}, A_{2}\right]-R\left(\xi_{1}, \xi_{2}\right)\right)
$$

as proved, for example, in $([39], \S 3)$.

Open Access. This article is distributed under the terms of the Creative Commons Attribution License (CC-BY 4.0), which permits any use, distribution and reproduction in any medium, provided the original author(s) and source are credited.

\section{References}

[1] J.M. Maldacena, The large-N limit of superconformal field theories and supergravity, Int. J. Theor. Phys. 38 (1999) 1113 [hep-th/9711200] [INSPIRE].

[2] W. Nahm, Supersymmetries and their representations, Nucl. Phys. B 135 (1978) 149 [INSPIRE]. 
[3] M.J. Duff and K.S. Stelle, Multimembrane solutions of D $=11$ supergravity, Phys. Lett. B 253 (1991) 113 [INSPIRE].

[4] G.W. Gibbons and P.K. Townsend, Vacuum interpolation in supergravity via super p-branes, Phys. Rev. Lett. 71 (1993) 3754 [hep-th/9307049] [INSPIRE].

[5] P.G.O. Freund and M.A. Rubin, Dynamics of dimensional reduction, Phys. Lett. B 97 (1980) 233 [INSPIRE].

[6] B.S. Acharya, J.M. Figueroa-O'Farrill, C.M. Hull and B.J. Spence, Branes at conical singularities and holography, Adv. Theor. Math. Phys. 2 (1999) 1249 [hep-th/9808014] [INSPIRE].

[7] D.R. Morrison and M.R. Plesser, Nonspherical horizons. 1., Adv. Theor. Math. Phys. 3 (1999) 1 [hep-th/9810201] [INSPIRE].

[8] P. de Medeiros, J. Figueroa-O'Farrill, S. Gadhia and E. Mendez-Escobar, Half-BPS quotients in M-theory: ADE with a twist, JHEP 10 (2009) 038 [arXiv:0909.0163] [INSPIRE].

[9] P. de Medeiros and J. Figueroa-O'Farrill, Half-BPS M2-brane orbifolds, Adv. Theor. Math. Phys. 16 (2012) 1349 [arXiv:1007.4761] [INSPIRE].

[10] J. Figueroa-O'Farrill and N. Hustler, The homogeneity theorem for supergravity backgrounds, JHEP 10 (2012) 014 [arXiv:1208.0553] [INSPIRE].

[11] N. Kowalsky, Noncompact simple automorphism groups of Lorentz manifolds and other geometric manifolds, Ann. Math. 144 (1996) 611.

[12] M. Deffaf, K. Melnick and A. Zeghib, Actions of noncompact semisimple groups on Lorentz manifolds, Geom. Funct. Anal. 18 (2008) 463.

[13] R. Slansky, Group Theory for Unified Model Building, Phys. Rept. 79 (1981) 1 [inSPIRE].

[14] G. Calvaruso, Homogeneous structures on three-dimensional Lorentzian manifolds, J. Geom. Phys. 57 (2007) 1279.

[15] B. Komrakov Jr., Four dimensional pseudo-riemannian homogeneous spaces. Classification of real pairs, International Sophus Lie Centre preprint, Moscow Russia (1995).

[16] J. Patera, R.T. Sharp, P. Winternitz and H. Zassenhaus, Continuous subgroups of the fundamental groups of physics. 3. The de Sitter groups, J. Math. Phys. 18 (1977) 2259 [INSPIRE].

[17] D.V. Alekseevsky, Homogeneous Lorentzian manifolds of a semisimple group, J. Geom. Phys. 62 (2012) 631 [arXiv:1101.3093].

[18] M.J. Duff, B.E.W. Nilsson and C.N. Pope, Kaluza-Klein supergravity, Phys. Rept. 130 (1986) 1 [INSPIRE].

[19] J.M. Figueroa-O'Farrill, P. Meessen and S. Philip, Homogeneity and plane-wave limits, JHEP 05 (2005) 050 [hep-th/0504069] [INSPIRE].

[20] A.L. Besse, Einstein Manifolds, Springer-Verlag, New York U.S.A. (1987).

[21] C. Chevalley and S. Eilenberg, Cohomology Theory of Lie Groups and Lie Algebras, Trans. Am. Math. Soc. 63 (1948) 85 [inSPIRE].

[22] E. Goursat, Sur les substitutions orthogonales et les divisions régulières de l'espace, Ann. Sci. École Norm. Sup. (3) 6 (1889) 9. 
[23] J.M. Figueroa-O'Farrill and G. Papadopoulos, Maximally supersymmetric solutions of ten-dimensional and eleven-dimensional supergravities, JHEP 03 (2003) 048 [hep-th/0211089] [INSPIRE].

[24] J. Figueroa-O'Farrill, Lie groups acting transitively (and isometrically) on anti de Sitter spaces, MathOverflow, http://mathoverflow.net/questions/78272 (2011).

[25] F. Englert, Spontaneous compactification of eleven-dimensional supergravity, Phys. Lett. B 119 (1982) 339 [INSPIRE].

[26] C.N. Pope and N.P. Warner, An SU(4) invariant compactification of $d=11$ supergravity on a stretched seven sphere, Phys. Lett. B 150 (1985) 352 [INSPIRE].

[27] C.N. Pope and N.P. Warner, Two new classes of compactifications of $d=11$ supergravity, Class. Quant. Grav. 2 (1985) L1 [InSPIRE].

[28] L. Castellani, L.J. Romans and N.P. Warner, A classification of compactifying solutions for $d=11$ supergravity, Nucl. Phys. B 241 (1984) 429 [InSPIRE].

[29] B. Biran, F. Englert, B. de Wit and H. Nicolai, Gauged $N=8$ supergravity and its breaking from spontaneous compactification, Phys. Lett. B 124 (1983) 45 [Erratum ibid. B 128 (1983) 461] [INSPIRE].

[30] M.A. Awada, M.J. Duff and C.N. Pope, $N=8$ Supergravity Breaks Down to $N=1$, Phys. Rev. Lett. 50 (1983) 294 [InSPIRE].

[31] F. Englert, M. Rooman and P. Spindel, Supersymmetry breaking by torsion and the Ricci flat squashed seven spheres, Phys. Lett. B 127 (1983) 47 [INSPIRE].

[32] M.J. Duff, B.E.W. Nilsson and C.N. Pope, Spontaneous supersymmetry breaking by the squashed seven sphere, Phys. Rev. Lett. 50 (1983) 2043 [Erratum ibid. 51 (1983) 846] [INSPIRE].

[33] F.A. Bais, H. Nicolai and P. van Nieuwenhuizen, Geometry of coset spaces and massless modes of the squashed seven sphere in supergravity, Nucl. Phys. B 228 (1983) 333 [INSPIRE].

[34] J.M. Figueroa-O'Farrill, F. Leitner and J. Simón, Supersymmetric Freund-Rubin backgrounds, Unfinished draft (2003).

[35] A. Lischewski, Computation of generalized Killing spinors on reductive homogeneous spaces, arXiv:1409.2664 [INSPIRE].

[36] D.P. Sorokin, V. Tkach and D. Volkov, Kaluza-Klein theories and spontaneous compactification mechanisms of extra space dimensions, in Proceedings of the third seminar on quantum gravity, Moscow Russia (1985), M.A. Markov, V.A. Berezin and V.P. Frolov eds., World Scientific, Singapore (1985).

[37] B. Kostant, Holonomy and the Lie algebra of infinitesimal motions of a riemannian manifold, Trans. Am. Math. Soc. 80 (1955) 528.

[38] R.P. Geroch, Limits of spacetimes, Commun. Math. Phys. 13 (1969) 180 [InSPIRE].

[39] J.M. Figueroa-O'Farrill, P. Meessen and S. Philip, Supersymmetry and homogeneity of M-theory backgrounds, Class. Quant. Grav. 22 (2005) 207 [hep-th/0409170] [INSPIRE].

[40] R. Bryant, Isometry group of a homogeneous space, MathOverflow, http://mathoverflow.net/questions/75887 (2011). 